\title{
Untersuchung der entzündlichen Komponente von Muskelschmerz nach exzentrischer Belastung bei jüngeren und älteren Erwachsenen
}

\author{
Dissertation zur Erlangung des akademischen Grades \\ Doctor medicinae (Dr. med.)
}

vorgelegt dem Rat der Medizinischen Fakultät

der Friedrich-Schiller-Universität Jena

von Steffen Koll

geboren am 04.12.1983

in Memmingen 
Erster Gutachter: Prof. Dr. Ulrich C. Smolenski, Jena Zweiter Gutachter: $\quad$ Prof. Dr. Georg Matziolis, Eisenberg Dritter Gutachter: Prof. Dr. Christoph C. Gutenbrunner, Hannover Tag der öffentlichen Verteidigung: 13.01.2014 


\section{ABKÜRZUNGSVERZEICHNIS}

\begin{tabular}{|c|c|c|}
\hline$A E$ & - & adverse event \\
\hline ALAT & - & Alanin-Aminotransferase \\
\hline$A S A T$ & - & Aspartat-Aminotransferase \\
\hline$B M I$ & - & body mass index \\
\hline $\mathrm{Cl}$ & - & Konfidenzintervall \\
\hline CK & - & Kreatinkinase \\
\hline $\operatorname{cox}$ & - & Cyclooxygenase \\
\hline$d$ & - & Tag/e \\
\hline$D F$ & - & degree of freedom \\
\hline DOMS & - & delayed-onset muscle soreness \\
\hline et al. & - & et alii \\
\hline GFR & - & glomeruläre Filtrationsrate \\
\hline GIT & - & Gastrointestinaltrakt \\
\hline$h$ & - & Stunde/n \\
\hline$H$ & - & Hypothese \\
\hline hs CrP & - & high-sensivity C-reaktives Protein \\
\hline IL & - & Interleukin \\
\hline ITT & - & intention-to-treat \\
\hline li. & - & linke/-es/-er \\
\hline LOCF & - & last observation carried forward \\
\hline M. & - & Musculus \\
\hline $\max$. & - & maximal/-e/-er/-es \\
\hline $\min$. & - & minimal/-e/-er/-es \\
\hline
\end{tabular}




\begin{tabular}{|c|c|c|}
\hline$m m H G$ & - & Millimeter Quecksilbersäule \\
\hline NRS & - & Numerische Rating Skala \\
\hline NSAR & - & nichtsteroidale Antirheumatika \\
\hline NYHA & - & New York Heart Association \\
\hline$p$ & - & probability \\
\hline$P G E_{2}$ & - & Prostaglandin $\mathrm{E}_{2}$ \\
\hline$P G F_{2 a}$ & - & Prostaglandin $\mathrm{F}_{2 a}$ \\
\hline$P P$ & - & per protocol \\
\hline PTT & - & partielle Thromboplastinzeit \\
\hline re. & - & rechte/-er/-es \\
\hline$R B E$ & - & repeated-bout effect \\
\hline$R R$ & - & Blutdruck \\
\hline$S A$ & - & Standardabweichung \\
\hline$S A E$ & - & severe adverse event \\
\hline SEM & - & standard error of the mean \\
\hline$T N F$ & - & Tumor Nekrose Faktor \\
\hline ZNS & - & zentrales Nervensystem \\
\hline$x^{2}$ & - & Chi-Quadrat \\
\hline
\end{tabular}




\section{INHALTSVERZEICHNIS}

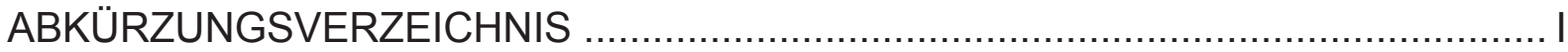

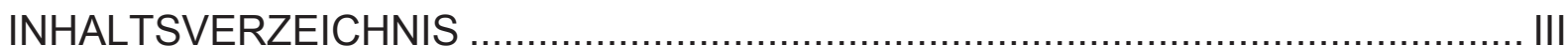

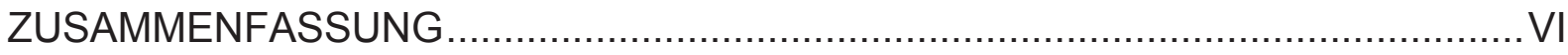

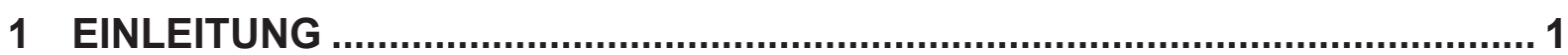

1.1 Grundlagen von Muskelschmerz nach exzentrischer Belastung ................. 1

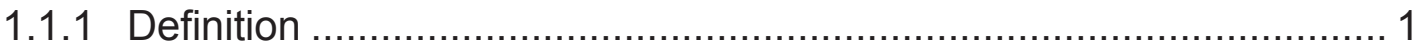

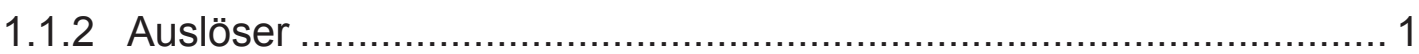

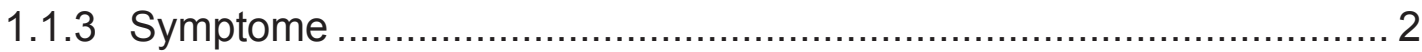

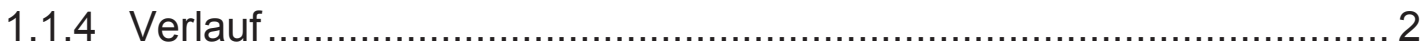

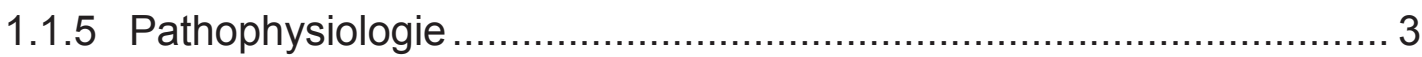

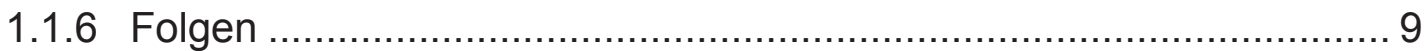

1.2 NSAR und Muskelschmerz nach exzentrischer Belastung ......................... 10

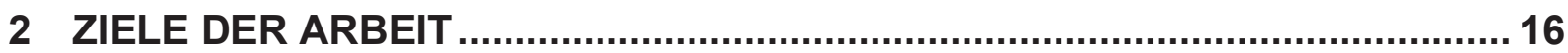

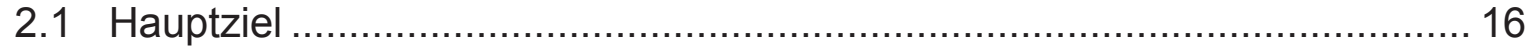

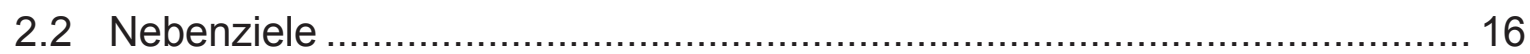

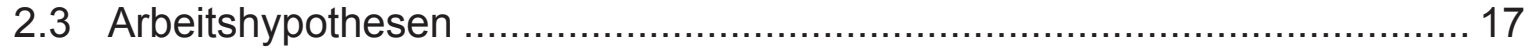

3 METHODIK

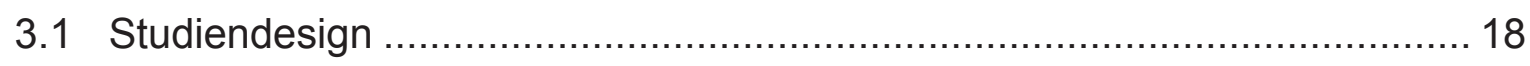

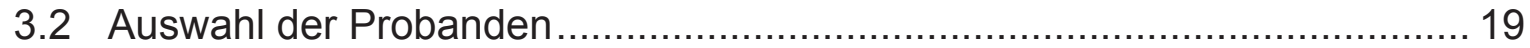

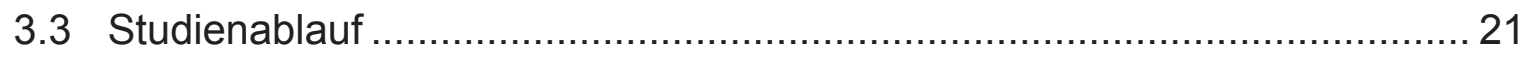

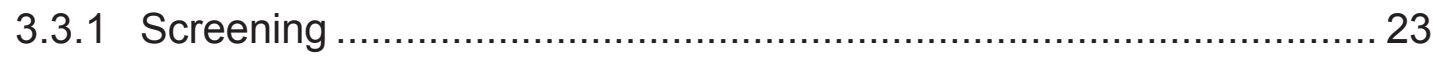

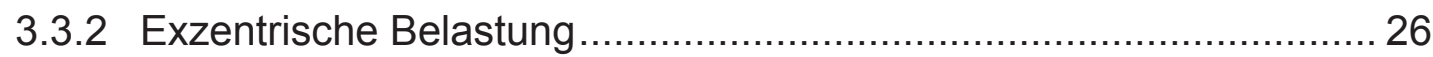

3.3.3 Nachbeobachtungsphase .................................................... 27 


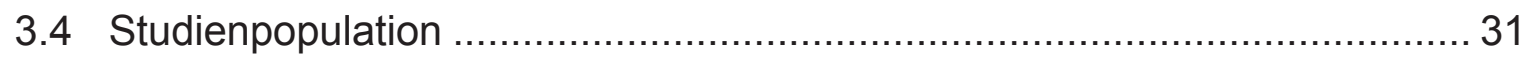

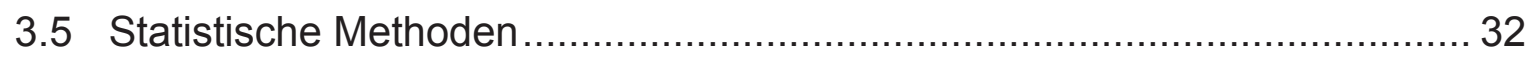

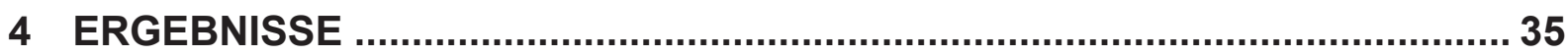

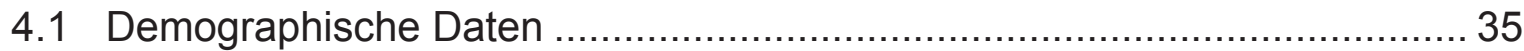

$4.2 \quad \mathrm{H}_{1}$ : Etoricoxib bei Muskelschmerz nach exzentrischer Belastung …............ 36

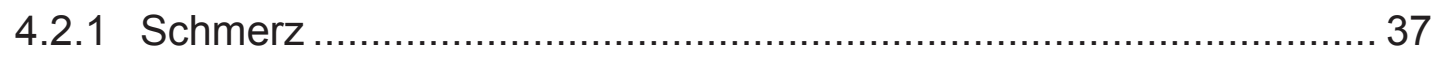

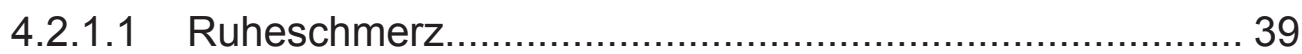

4.2.1.2 Bewegungsschmerz .............................................. 42

4.2.1.3 Schmerzschwelle am max. Druckpunkt.......................... 44

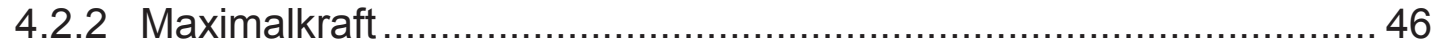

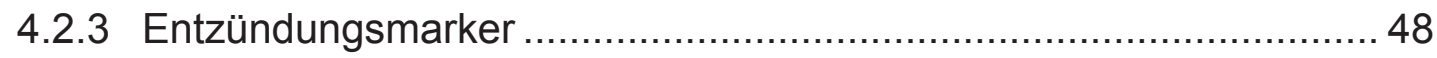

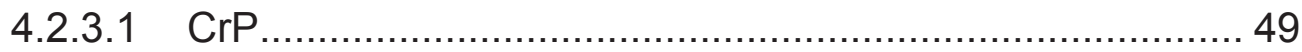

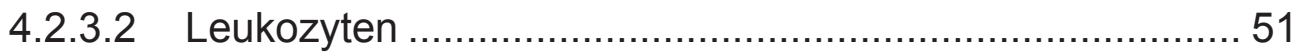

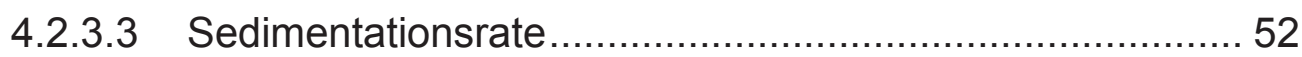

4.3 $\mathrm{H}_{3}$ : Alter und Muskelschmerz nach exzentrischer Belastung .....................53

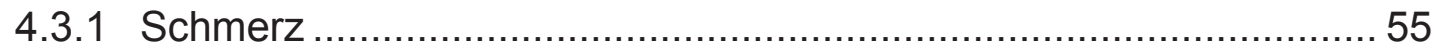

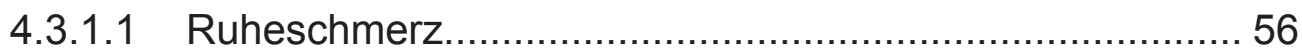

4.3.1.2 Bewegungsschmerz ................................................. 57

4.3.1.3 Schmerzschwelle am max. Druckpunkt........................... 58

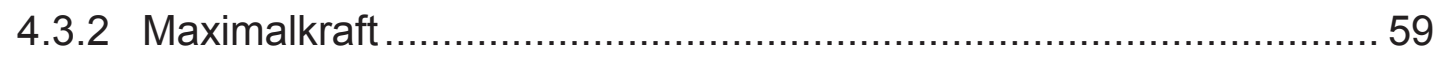

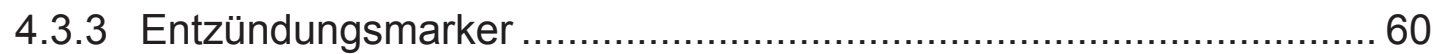

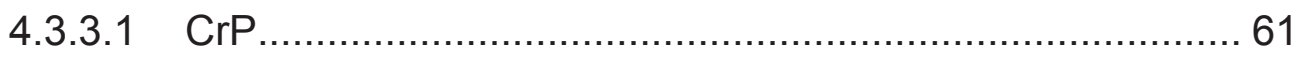

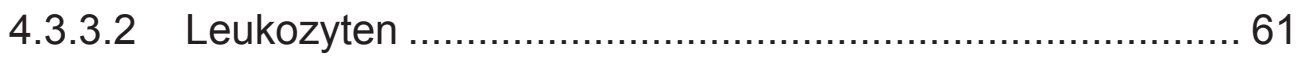

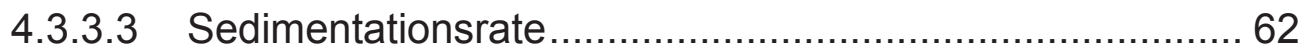

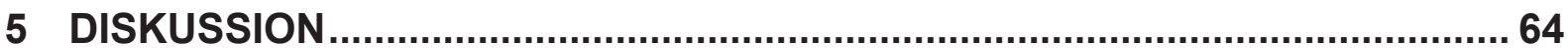


5.1 Studienmodell

$5.2 \mathrm{H}_{1}$ : Etoricoxib bei Muskelschmerz nach exzentrischer Belastung ............. 68

$5.3 \mathrm{H}_{2}$ : Einfluss der Studienmethodik auf Ergebnisse bisheriger Studien......... 74

$5.4 \mathrm{H}_{3}$ : Alter und Muskelschmerz nach exzentrischer Belastung.................... 82

$5.5 \quad \mathrm{H}_{4}$ : NSAR bei Muskelschmerz nach exzentrischer Belastung .................... 86

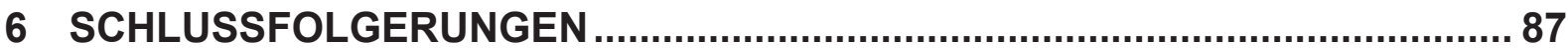

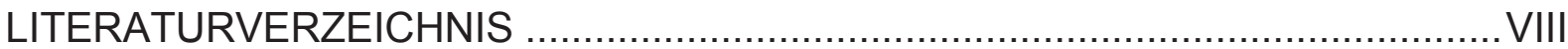

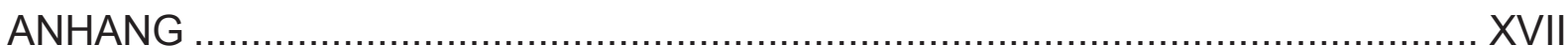

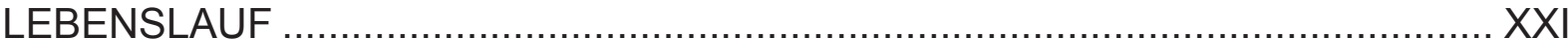

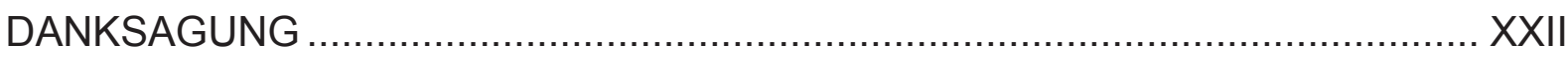

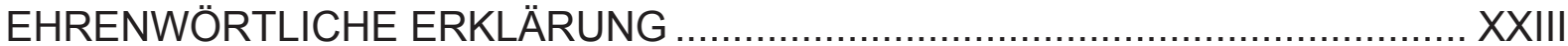




\section{ZUSAMMENFASSUNG}

Wissenschaftlicher Hintergrund: Exzentrische Belastung der Muskulatur verursacht einen verzögert auftretenden Muskelschmerz, der von Muskelschwellung, Muskelsteifheit, eingeschränkter Beweglichkeit und Muskelkraftverlust begleitet wird - das Muskelkatersyndrom (DOMS). Die durch exzentrische Kontraktion verursachten mikroskopischen Schäden in der Muskulatur führen unter anderem zur Aktivierung einer akuten Entzündungsreaktion. Diese wird als einer der zugrunde liegenden Mechanismen in der Entstehung von DOMS betrachtet und dient dem Reparaturprozess im Muskel. Zur Behandlung von Muskelschmerz nach exzentrischer Belastung wird häufig auf eine antientzündliche Medikation mit nichtsteroidalen Antirheumatika (NSAR) zurückgegriffen. Deren Wirksamkeit in der Therapie von DOMS wird in Studien jedoch kontrovers diskutiert.

Fragestellung und Ziele: Diese Arbeit untersucht die Wirksamkeit antientzündlicher Therapie bei Muskelschmerz nach exzentrischer Belastung. Dadurch werden Aussagen zur Bedeutung der Entzündungsreaktion für die Entstehung und den Verlauf von DOMS ermöglicht. Zudem wird der Einfluss des Alters auf die Entwicklung von DOMS untersucht.

Methodik: In dieser prospektiven, randomisierten, doppelblinden (probanden- und untersucherverblindeten), placebo-kontrollierten, crossover-Studie wurde der Effekt einer antientzündlichen Therapie mit dem COX-2-spezifischen NSAR Arcoxia* (Etoricoxib) auf den Verlauf von DOMS nach exzentrischer Belastung der Knieextensoren bei 25 jüngeren und 25 älteren Erwachsenen untersucht. $16 \pm 2 \mathrm{~h}$ nach der Erzeugung von DOMS in der Oberschenkelmuskulatur eines Beines auf dem "extrafit Beinstrecker" wurde mit der täglichen Einahme von entweder 90 mg Etoricoxib oder Placebo für jeweils 7 Tage begonnen. Die Auswirkung der Behandlung auf den Muskelschmerz in Ruhe und während Bewegung, auf die Wiederherstellung der Muskelkraft, auf die Hitzeschmerzschwelle am druckempfindlichsten Punkt des Muskelkaters sowie auf die Entzündungsmarker im Blut wurde $0 \mathrm{~h}, 1 \mathrm{~h}$, 8 h, 24 h, 48 h, 72 h, 96 h, 120 h, 144 h und 168 h nach der exzentrischen Bela- 
stung untersucht. Durch die Wiederholung desselben Prozederes am kontralateralen Bein in einer zweiten Versuchsphase diente jeder Proband als seine eigene Kontrolle.

Ergebnisse und Diskussion: Durch das Studienmodell wurde DOMS in statistisch signifikanter Ausprägung erzeugt. 16-40 h nach Belastung war der Muskelschmerz maximal ausgeprägt und eine Abnahme der maximalen Muskelkraft von bis zu $30 \%$ wurde beobachtet. Etoricoxib konnte die nach exzentrischer Belastung ablaufende Entzündungsreaktion im Vergleich zu Placebo signifikant abschwächen ( $p=$ 0,0341). Bezüglich der Muskelschmerzen zeigten sich keine signifikanten Behandlungseffekte von Etoricoxib im Vergleich zu Placebo ( $p=0,7257)$. In der frühen Phase der Behandlung (während der ersten $24 \mathrm{~h}$ ) bestand ein nicht signifikanter Trend zur Reduktion des Muskelschmerzes durch Etoricoxib ( $p=0,1341$ ). Hinsichtlich der Maximalkraft und der Hitzeschmerzschwelle zeigten sich keine signifikanten Behandlungsunterschiede zwischen Etoricoxib und Placebo ( $p=0,6575$, bzw. $p=0,4586)$. Wenn überhaupt, so scheint die Regeneration der Muskelkraft und die Wiederherstellung der ursprünglichen Schmerzschwelle in der Erholungsphase nach exzentrischer Belastung durch Etoricoxib verzögert zu werden. Keine behandlungsbezogenen, wohl aber physiologisch relevante altersbezogene Unterschiede zwischen jüngeren und älteren Erwachsenen wurden beobachtet. Ältere Erwachsene beklagten weniger Muskelschmerz (signifikant höhere Schmerzschwelle, $p=0,0147$ ) und benötigten mehr Zeit zur Regeneration nach exzentrischer Belastung (signifikant längeres Bestehen von Muskelschmerz $(p=0,016)$ und reduzierter Muskelkraft $(p=0,0584))$ als jüngere Erwachsene.

Schlussfolgerungen: Die akute Entzündungsreaktion im Rahmen von DOMS spielt nicht nur in der Entstehung des Muskelschmerzes eine wichtige Rolle, sondern scheint auch für den Regenerationsprozess der Muskulatur essentiell zu sein. Von einer unkritischen NSAR-Einnahme bei Muskelschmerz nach exzentrischer Belastung sollte abgeraten werden. Eine durch die antientzündliche Wirksamkeit der NSAR bedingte mögliche Verzögerung der muskulären Regeneration könnte den scheinbar schmerzlindernden Effekt überwiegen. 


\section{EINLEITUNG}

\subsection{Grundlagen von Muskelschmerz nach exzentrischer Belastung}

\subsubsection{Definition}

Muskelschmerz nach ausgeprägter Belastung der Muskulatur erfordert eine differenzierte Betrachtung. Miles und Clarkson unterschieden drei Typen von Muskelschmerz, die durch unterschiedliche Ätiologien und Zeitverläufe charakterisiert sind: 1. Schmerz während oder unmittelbar nach Belastung, 2. Schmerz durch Muskelkrämpfe sowie 3. „delayed onset muscle soreness“ (DOMS) (Miles und Clarkson 1994).

DOMS beschreibt einen nach exzentrischer Belastung verzögert auftretenden Muskelschmerz, der von Muskelschwellung, Muskelsteifheit, eingeschränkter Beweglichkeit und Muskelkraftverlust begleitet wird. Im allgemeinen Sprachgebrauch wird dieser Zustand als „Muskelkater“ bezeichnet (Böning 2002).

\subsubsection{Auslöser}

Generell kann jeder, ob Untrainierter oder Spitzensportler, DOMS entwickeln (Steinmann und Allwang 2008). DOMS wird durch exzentrische Belastung der Muskulatur ausgelöst. Es gibt bestimmte Situationen nach denen DOMS typischerweise beobachtet wird. Eine dieser und jedem wohl bekannte Situation ist die ungewohnte körperliche Belastung, z. B. die Ausführung nicht eingeübter Bewegungen im Alltag, im Rahmen einer neuen Sportart oder aber die Wiederaufnahme des Trainings nach längerer Verletzungspause. Weiterhin wird das Auftreten von DOMS nach außergewöhnlich starker Belastung der Muskulatur z. B. nach sportlichen Wettkämpfen, nach tonisch-klonischen Krämpfen im Rahmen einer Epilepsie sowie nach der Anwendung von Muskelrelaxantien in der Anästhesie beschrieben. Gemeinsamkeit dieser Situationen ist eine unvollkommene intramuskuläre Koordination (Böning 2002). 


\subsubsection{Symptome}

Das Hauptsymptom von DOMS ist der Muskelschmerz. Es besteht eine erhöhte Schmerzempfindlichkeit der betroffenen Muskulatur insbesondere während Bewegungen, bei Palpation, sowie auf andere physikalische Reize. Zunächst ist vor allem der distale Muskelabschnitt am Übergang von Muskel zur Sehne betroffen, da in diesem Bereich hohe Konzentrationen von Schmerzrezeptoren im Bindegewebe vorliegen (Newham et al. 1982). Außerdem zeigen die Muskelfasern im Bereich der Insertion in die Sehne durch ihre schräg verlaufende Anordnung eine reduzierte Widerstandsfähigkeit auf Zugkräfte und damit eine erhöhte Anfälligkeit für mikroskopische Muskelschäden (Noonan und Garrett 1992). Nach einiger Zeit breitet sich der Schmerz weiter aus und bezieht in der Regel den gesamten Muskel mit ein (Maclntyre et al. 1995).

Weitere Symptome von DOMS sind erhöhte Muskelspannung durch Schwellung, erhöhte Muskelrigidität, Kraftverlust und eingeschränkter Bewegungsradius der Gelenke (McHugh et al. 1999).

\subsubsection{Verlauf}

Der Muskelschmerz beginnt 6-8 Stunden nach der exzentrischen Belastung, ist nach 24-48 Stunden maximal ausgeprägt (Crameri et al. 2001, Connolly et al. 2003, Dartnall et al. 2008, Dundon et al. 2008) und nimmt danach wieder kontinuierlich ab. In der Regel besteht die Schmerzsymptomatik - je nach Ausprägung von DOMS - über einen Zeitraum von 5-8 Tagen (Bottas et al. 2009, Philippou et al. 2009, Rother et al. 2009).

Der Verlust der Maximalkraft der betroffenen Muskulatur ist direkt nach der exzentrischen Belastung am ausgeprägtesten (Smith 1991). Innerhalb der ersten 48 Stunden nach Belastung wurde eine Abnahme der Maximalkraft von bis zu 30$60 \%$ beobachtet (Dudley et al. 1997). Die vollständige Wiederherstellung der ursprünglichen Muskelkraft braucht in der Regel länger als 5 Tage und kann mehr als 2 Wochen in Anspruch nehmen (Newham et al. 1987). 
Die intramuskuläre Schwellung erreicht 3-4 Tage nach Belastung ihren Gipfel und ist innerhalb von 10 Tagen vollständig rückläufig (Cleak und Eston 1992).

\subsubsection{Pathophysiologie}

Schon seit vielen Jahren ist DOMS Gegenstand der Forschung. Vor allem seit den 1980er Jahren wurden vermehrt Studien durchgeführt, die darauf abzielten, Ursachen und Entstehungsmechanismen des nach Belastung verzögert auftretenden Muskelschmerzes zu erforschen und die Pathophysiologie von DOMS zu verstehen. Gemeinsamer Zweck dieser Studien war es, aus den gewonnenen Erkenntnissen geeignete Präventions- und Therapiestrategien abzuleiten. Im Laufe der Zeit entstanden verschiedene Theorien und Erklärungsmodelle, die in ihrer Gesamtheit betrachtet zum aktuellen Verständnis von DOMS beitragen.

In den 1970er Jahren bestand die Annahme, dass sich während und nach ausgeprägter muskulärer Belastung erhebliche Mengen an Laktat und anderen toxischen Stoffwechselendprodukten im Muskel anreichern, die die Nozizeptoren reizen und auch noch einige Zeit nach Belastung Schmerz auslösen können (Wietoska und Böning 1979). Es wurde angenommen, dass die anfallenden Metabolite zu Vasodilatation und interzellulärem Ödem führen, in dessen Folge die Blutversorgung der Muskulatur behindert und die Hypoxie verstärkt wird. Zusammen mit der erhöhten Gewebespannung werden durch die Hypoxie Nozizeptoren gereizt und Muskelschmerz verursacht, der reflektorisch zu Muskelspasmus und erhöhtem Muskeltonus führt. Es kommt zu einem Teufelskreis von Ischämie, Hypoxie und Muskelspasmus, der über Tage andauern kann (de Vries 1966). Diese „Muskelspasmus-Theorie“ wurde jedoch weitgehend verlassen, da gezeigt werden konnte, dass während exzentrischer Belastung erheblich weniger Laktat anfällt als während konzentrischer Belastung, welche nicht mit dem Auftreten von DOMS assoziiert ist (Schwane et al. 1983).

Seit gezielten, auf Muskelbiopsien gestützten Studien in den 1980er Jahren, steht aktuell die Theorie, dass durch exzentrische Muskelkontraktionen verursachte mikroskopische Schäden in der Muskulatur ursächlich für die Entstehung von DOMS 
sind, im Vordergrund der wissenschaftlichen Betrachtung. Schon Anfang des 20. Jahrhunderts wurde in einem Artikel das Auftreten von Muskelschmerz nach rhythmischer Belastung der Finger beschrieben und als Ursache dafür „Risse im Muskel" vermutet (Hough 1902). In den 1960er Jahren wurde erstmals gezeigt, dass lediglich durch die exzentrische Form der Muskelkontraktion DOMS erzeugt werden konnte (Asmussen 1956).

Die exzentrische Muskelkontraktion unterscheidet sich in einigen wichtigen Punkten von anderen Formen der Muskelkontraktion. Sie ist primär dadurch gekennzeichnet, dass der Muskel kontrahiert, um einer Dehnung durch externe Kräfte entgegenzuwirken, wie z. B. beim Treppabsteigen, oder dass der bereits kontrahierte Muskel durch externe Kräfte gedehnt wird, wie z. B. bei der Kniebeuge. Einerseits durch die elastischen Rückstellkräfte von Sehnen, Bindegewebe und Muskelfasern selbst - genannt seien u. a. Titin und die Elastiziität der Hälse von Myosinköpfchen - andererseits durch die Bildung von Aktin-Myosin-Brücken wird die Verlängerung des Muskels abgebremst. Der Muskel leistet somit negative Arbeit (Böning 2011). Zudem sind im Vergleich zu konzentrischen und isometrischen Kontraktionsformen die subjektive Belastung, die Ermüdung und die Schmerzwahrnehmung während der exzentrischen Kontraktion geringer (Newham et al. 1983). Elektromyographische Untersuchungen zeigten des weiteren, dass während der exzentrischen Muskelkontraktion eine deutlich höhere Maximalkraft erreicht wird (Semmler et al. 2007, Dartnall et al. 2008). Dabei besteht ein niedrigerer metabolischer Umsatz im Muskel. Im Vergleich zu konzentrischen Kontraktionen ist der Sauerstoffverbrauch während exzentrischen Kontraktionen um das 8-fache erniedrigt und deutlich weniger ATP wird verbraucht. Insgesamt werden bei exzentrischen Kontraktionen weniger motorische Einheiten rekrutiert, wodurch die Zugbelastung auf der einzelnen Muskelfaser erhöht ist (Seichert 2003). Brunello et al. zeigten, dass während exzentrischer Kontraktionen beide Köpfchen des Myosinfilaments an Aktin binden. Dadurch kann unter einem geringeren ATP-Verbrauch eine deutlich stärkere Aktin-Myosin-Brücken-Bindung hergestellt werden (Brunello et al. 2007). 
Im Bestreben die Länge des Sarkomers gegen die externe Dehnung konstant zu halten, führen die Myosinköpfchen auf beiden Seiten der Z-Linien gegensätzliche Zugbewegungen aus. Muskeldehnungen auf mehr als 140\% der optimalen Länge übersteigen jedoch die mechanische Widerstandsfähigkeit der Sarkomere (Faulkner et al. 1993). Morgan und Allen nahmen an, dass rasche Verlängerungen über die optimale Länge hinaus eine uneinheitliche Überdehnung der Sarkomere bewirken. Die kontraktilen Elemente werden so weit auseinandergezogen, dass sie ab einem gewissen Punkt nicht mehr überlappen. Die Sarkomere werden nur noch durch passive Elemente zusammengehalten und zerreißen im Bereich ihrer mechanischen Schwachstelle, der Z-Linie (Morgan und Allen 1999).

Diese Schäden in der Mikrostruktur der Muskulatur konnten Fridén et al. in Studien nachweisen, bei denen sie Probanden im Anschluss an exzentrische Belastungen auf dem Fahrradergometer Muskelbiopsien entnahmen und diese im Elektronenmikroskop untersuchten. Sie zeigten, dass durch exzentrische Belastung Z-Linien überdehnt, auseinandergerissen und zum Teil vollständig zerstört werden. Die angrenzenden Myofilamente zeigen dabei Auflösungserscheinungen und Desorganisation. Desmin wird zerstört. Diese mikroskopischen Strukturschäden waren eine Stunde nach Belastung in 32\%, 3 Tage nach Belastung in 52\% und 6 Tage nach Belastung in 12\% der entnommenen Proben vorhanden (Fridén et al. 1983). Vor allem schnell kontrahierende Muskelfasern vom Typ-II („fast-twitch-fibres“) waren von der Schädigung betroffen (Lieber et al. 1991, Choi et al. 2012). Grund hierfür scheint eine erhöhte strukturelle Labilität der Typ-II Fasern bei Belastung sowie eine selektive Rekrutierung dieses Fasertyps für exzentrische Kontraktionen zu sein (Connolly et al. 2003).

Newham et al. führten dazu analoge Studien durch. Sie verglichen die strukturellen Veränderungen innerhalb der Sarkomere nach rein exzentrischer Belastung mit denen nach rein konzentrischer Belastung. Lediglich die exzentrisch belasteten Muskeln wiesen in Biopsien o. g. Strukturschäden auf. Diese Veränderungen waren direkt nach der Belastung sichtbar und 24 sowie 48 Stunden nach Belastung weiter progredient (Newham et al. 1983). 
Diese Studien demonstrierten ausnahmslos, dass die strukturellen Schäden in den Muskelfasern direkt durch die exzentrische Belastung induziert wurden. Außerdem zeigten sie, dass das Ausmaß der Mikroschäden in den folgenden Stunden zunahm und weitere Bereiche der Muskulatur miteinbezog. Als einer der entscheidenden Faktoren in diesem Prozess wurden $\mathrm{Ca}^{2+}$-Ionen genannt (Gissel 2000). Neben Schäden an kontraktilen Elementen und am Zytoskelett der Muskelfasern treten Veränderungen im Bereich des Sarkolemms und des sarkotubulären Systems auf (Fridén und Lieber 2001). Mechanische Zerstörung oder Lyse der Zellmembranen von Sarkolemm und sarkoplasmatischem Retikulum erhöhen dessen Permeabilität und ermöglichen die Diffusion intra- und extrazellulärer Stoffe und Strukturen durch die geschädigte Barriere. Armstrong schlussfolgerte, dass $\mathrm{Ca}^{2+}$ Ionen intrazellulär akkumulieren, Proteasen und Phospholipasen aktivieren, die den Prozess der Zerstörung der Muskelfeinstruktur in den Stunden nach exzentrischer Belastung weiter vorantreiben (Armstrong 1990). Eine erhöhte Aktivität dieser hydrolytischen Enzyme wiesen Fridén und Lieber nach, indem sie eine erhöhte Anzahl an Lipofuscin-Granula in Muskelbiopsaten 3 Tage nach exzentrischer Belastung beobachteten (Fridén und Lieber 1992).

Eine weitere Folge der aufgelösten Integrität zellulärer Kompartimente im geschädigten Muskel ist die Diffusion muskelspezifischer intrazellulärer Stoffe, wie z. B. Kreatinkinase (CK), Lactat-Dehydrogenase, Hydroxyprolin, Hydroxilysin oder Myoglobin in den Blutkreislauf. Insbesondere CK wird als indirekter Marker im Blut genutzt, um das Ausmaß des Muskelschadens nach exzentrischer Belastung zu beschreiben. Mehrere Forschungsgruppen zeigten in ihren Studien einen Anstieg der CK direkt nach exzentrischer Belastung mit einem Gipfel nach 48 Stunden (Twist und Eston 2005, Tofas, et al. 2008, Philippou et al. 2009).

Strauber et al. zeigten, dass neben den Muskelfasern auch das muskuläre Bindegewebe nach exzentrischer Belastung Veränderungen aufweist. Mastzelldegenerationen, Anhäufungen von mononukleären Zellen, Albumin und Fibrinogen, sowie Veränderungen der Kapillaren werden in Peri- und Endomysium geschädigter 
Muskeln beschrieben. Weiterhin entstehen zwischen Bindegewebe und Muskelfaser erweiterte interzelluläre Räume (Stauber et al. 1990).

Diese strukturellen Schäden der Muskulatur führen zur Aktivierung einer Entzündungsreaktion im Muskel, die dem Reparatur- und Regenerationsprozess dient (Clarkson und Hubal 2002). Initial werden die Muskelschäden durch die während der Entzündungsreaktion ablaufenden Prozesse noch erweitert (Maclntyre et al. 1995). Die Koordination zwischen entzündungsbedingter Schädigung und Regeneration wird durch proinflammatorische (u.a. IL-1 $\beta$, IL-6, TNF, Interferon- $\alpha$ ) und antiinflammatorische Zytokine (z.B. IL-10) gesteuert (Philippou et al. 2009). Interleukin-1 $\beta$ (IL-1 $\beta$ ) steigt im Muskel direkt nach exzentrischer Belastung an und induziert die Chemotaxis und Adhäsion von neutrophilen Granulozyten. Neutrophile sind die ersten Zellen, die in die geschädigten Bereiche einwandern. Sie sind bereits 45 Minuten nach Belastung im Muskel erhöht und auch noch 5 Tage später nachweisbar (Fielding et al. 1993). Auch Maclntyre et al. beobachteten eine Migration von Neutrophilen in den geschädigten Muskel 2-4 Stunden nach exzentrischer Belastung (Maclntyre et al. 2000). Dort phagozytieren sie abgestorbene Gewebsbestandteile indem sie u. a. Superoxide, Proteasen und freie Radikale freisetzen. Während hoher Belastung bilden neben den Neutrophilen auch Xanthin-Oxidasen vermehrt freie Radikale im Muskel. Überschreitet diese Produktion die Abwehrkapazität von zellulären Scavenger- und Antioxidanz-Systemen, können die freien Radikale Muskelfasern und Zellmembranen weiter schädigen (Sjödin et al. 1990).

Die von den Neutrophilen freigesetzten Stoffe wirken chemotaktisch auf weitere Entzündungszellen. Einige Stunden nach der mechanischen Muskelverletzung werden Monozyten angelockt und wandern in die geschädigten Bereiche ein. Im Gewebe reifen sie zu Makrophagen, die nekrotisches Gewebe und Fremdkörper beseitigen (Ryan und Majno 1977). Makrophagen und weitere Substanzen wie Interleukin-I und $\mathrm{Ca}^{2+}$-Ionen synthetisieren im entzündeten Gewebe große Mengen an Prostaglandin $E_{2}\left(P_{G}\right)$. Smith konnte eine signifikante Erhöhung der $\mathrm{PGE}_{2^{-}}$ Konzentration 24 Stunden nach exzentrischer Belastung nachweisen. PGE 2 setzt durch eine Sensibilisierung von Nozizeptoren die Schmerzschwelle herab. In die- 
sem Zustand der Hyperalgesie können schon einfache, normalerweise unterschwellige chemische, mechanische oder thermische Stimuli Schmerzfasern aktivieren und somit Muskelschmerz auslösen (Smith 1991).

Ein bedeutender Faktor in dieser Schmerzrezeptorstimulation ist sicherlich das Gewebsödem. In einer Studie von Bobbert et al. war 24, 48 und 72 Stunden nach exzentrischer Belastung der Umfang der betroffenen Extremität im Seitenvergleich deutlich vergrößert (Bobbert et al. 1986). Shellock et al. beobachteten im MRT Flüssigkeitsansammlungen im Muskel nach exzentrischer Belastung (Shellock et al. 1991). Bindegewebsschäden, Auflösung von Muskelzellen, entzündungsbedingte Vasodilatation und Erhöhung der Kapillarpermeablilität bewirken die Ansammlung von Exsudat im Gewebe (Clarkson und Hubal 2002). Es entsteht eine mess- und tastbare Schwellung der Muskulatur und ein damit erhöhter Gewebedruck (Fridén et al. 1986). Typischerweise ist die exzentrisch belastete Muskulatur besonders schmerzhaft, wenn sich der intramuskuläre Druck noch weiter erhöht, z. B. bei Palpation oder während Kontraktion. Ein hoher Gewebedruck stellt einen mechanischen Stimulus für die $\mathrm{PGE}_{2}$-sensibilisierten Nozizeptoren dar und spielt somit eine wichtige Rolle bei der Schmerzentstehung nach exzentrischer Muskelbelastung (Smith 1991).

Nach aktueller Studienlage wird der Muskelschaden nach exzentrischer Belastung als Ursprung jeglicher ablaufender Prozesse im Muskel betrachtet. Es gab jedoch auch Kritik und Zweifel an Methoden, Ergebnissen und resultierenden Schlussfolgerungen einiger Studien, die zu der gegenwärtigen Sichtweise beigetragen haben. Malm et al. machten z. B. auf die Möglichkeit aufmerksam, dass Muskelbiopsien selbst Schäden in der Mikrostruktur des Muskels mit anschließender Infiltration von Entzündungszellen verursachen können (Malm et al. 2000). Der genaue zeitliche Ablauf von DOMS und die Rolle der dabei ablaufenden Entzündungsreaktion ist weiterhin nicht abschließend geklärt. Sicher scheint jedoch, dass alle o. g. Hypothesen zur Entstehung von DOMS eine Rolle spielen und somit in ihrer Gesamtheit und nicht isoliert betrachtet werden sollten (Cheung et al. 2003). 


\subsubsection{Folgen}

DOMS ist ein selbstlimitierender Prozess. Die Veränderungen und damit verbundenen Symptome beginnen und verschwinden in einem typischen zeitlichen Verlauf, ohne dass ein Schaden zurückbleibt (restitutio ad integrum) (Maclntyre et al. 1995). Die Regenerationsphase im Muskel läuft zeitlich überlappend mit der Entzündungsreaktion $a b$ und beginnt innerhalb der ersten Woche nach exzentrischer Belastung (Kaariainen et al. 2000). An der Regeneration maßgeblich beteiligte Zellen, wie Satellitenzellen oder Muskelstammzellen, werden im Bereich der Muskelverletzung freigesetzt (Rantanen et al. 1995). Unter dem Einfluss von Prostaglandinen, die während der Entzündungsreaktion produziert werden, werden verschiedene Wachstumsfaktoren im Muskel freigesetzt. Diese aktivieren und stimulieren die Myoblastenproliferation und -differenzierung sowie die Muskelproteinsynthese und -hypertrophie (Prisk und Huard 2003). Histologisch sind erste Anzeichen des Regenerationsprozesses durch das vermehrte Auftreten von Fibroblasten im geschädigten Muskel 72 Stunden nach exzentrischer Belastung sichtbar (Jones et al. 1986).

Im Rahmen der Regeneration nach exzentrischer Belastung findet eine zelluläre Adaptation im Muskel statt. Die Anpassung des Muskels an die stattgehabte exzentrische Belastung ist der Grund für ein abgeschwächtes oder ausbleibendes Auftreten von DOMS nach gleichartigen exzentrischen Belastungen, die innerhalb von 6 Monaten auf die erste folgen (Nosaka et al. 2001). Diese Anpassungsreaktion wird als „repeated-bout effect" (RBE) bezeichnet (Lavender und Nosaka 2008). Der RBE ist abhängig vom Auftreten einer Entzündungsreaktion. Lapointe et al. wiesen nach, dass eine Hemmung der Entzündung durch Einnahme von Diclofenac die Entwicklung des RBEs nach exzentrischer Belastung verhinderte (Lapointe et al. 2002).

Smith vermutet, dass der Muskelschmerz und die Einschränkungen der Beweglichkeit während einer kritischen Phase des Heilungsprozesses auftreten, um 
durch „freiwillige Inaktivität“ die Bedingungen für den Regenerationsprozess zu optimieren (Smith 1991). Clancy und Clarkson zeigten, dass durch Immobilisation die Regeneration der Muskelkraft beschleunigt wird (Clancy und Clarkson 1990).

Einige Studien wiesen nach, dass während DOMS die Muskelreflexantwort reduziert (Hedayatpour et al. 2011) und die Propriozeption herabgesetzt ist (Coudreuse et al. 2004). Aus diesem Grund ist während des Bestehens von DOMS die Wahrscheinlichkeit eine Sportverletzung zu erleiden erhöht (Cheung et al. 2003).

\section{$1.2 \quad$ NSAR und Muskelschmerz nach exzentrischer Belastung}

In der allgemeinen Praxis werden zur Behandlung von Muskelschmerz nach exzentrischer Belastung häufig nichtsteroidale Antirheumatika (NSAR) eingesetzt. In Hinblick auf die Entzündungsreaktion, die im Rahmen von DOMS abläuft, erscheint ein solcher Behandlungsversuch als sinnvoll. In den letzten Jahren wurde eine ganze Reihe von Studien publiziert, die den Effekt von NSAR auf Muskelschmerz nach exzentrischer Belastung untersucht haben. Dabei zeigten sich mitunter deutlich unterschiedliche Ergebnisse. Eine Wirksamkeit von NSAR in der Therapie von DOMS wird daher weiterhin kontrovers diskutiert.

Mehrere Studien zeigten eine mangelnde Wirksamkeit von Flurbiprofen in der Behandlung von DOMS (Kuipers et al. 1985, Semark et al. 1999).

Howell et al. beobachteten in ihrer Studie sogar einen negativen Effekt von Flurbiprofen. Sie ließen 44 Probanden in drei Durchgängen exzentrische Kontraktionen der Ellenbogenflexoren bis zur Ermüdung ausführen. 12 Stunden vor der Belastung sowie über die folgenden 14 Tage nach Belastung wurden täglich $100 \mathrm{mg}$ Flurbiprofen (oral) eingenommen. Die Entwicklung und Ausprägung von Muskelschmerz und -schwellung zeigten keinen Unterschied im Vergleich zur PlaceboGruppe. In der Flurbiprofen-Gruppe wurde jedoch eine verlangsamte Erholung der maximalen Muskelkraft beobachtet (Howell et al. 1998).

Im Rahmen einer Vorstudie verabreichten Hasson et al. 20 Probanden einmalig 400 mg Ibuprofen (oral) 4 Stunden vor oder 24 Stunden nach exzentrischer Bela- 
stung. Unter der Behandlung verspürten die Probanden signifikant weniger Muskelschmerz und zeigten eine signifikant geringere Abnahme der maximalen Muskelkraft (Hasson et al. 1993).

Tokmakidis et al. induzierten bei 19 Probanden DOMS durch exzentrische Kontraktionen der Kniebeugemuskulatur. Über einen Zeitraum von 48 Stunden wurden alle 8 Stunden 400 mg Ibuprofen (oral) verabreicht. Unter der Therapie mit Ibuprofen zeigten die Probanden signifikant weniger Muskelschmerz nach 24 Stunden als die Placebo-Gruppe. Hinsichtlich der maximalen Muskelkraft und des Bewegungsradius des Gelenkes konnten keine Unterschiede demonstriert werden (Tokmakidis et al. 2003).

Rahnama et al. ließen in ihrer Studie 44 Probanden 70 exzentrische Kontraktionen des M. biceps brachii ausführen, um DOMS zu erzeugen. Eine Stunde vor der Belastung begann die Behandlung mit 400 mg Ibuprofen (oral) dreimal täglich, die über 48 Stunden fortgeführt wurde. Der Muskelschmerz war 48 Stunden nach der Belastung im Vergleich zur Kontrollgruppe signifikant erniedrigt (Rahnama et al. 2005).

Außer in diesen drei Publikationen konnte in keiner der durchgeführten Studien eine Wirksamkeit von Ibuprofen bei Muskelschmerz nach exzentrischer Belastung nachgewiesen werden. Die überwiegende Mehrheit der Studien zeigte keinen Behandlungseffekt für Ibuprofen (Donnelly et al. 1990, Grossmann et al. 1995, Howell et al. 1998, Pizza et al. 1999, Stone et al. 2002, Arendt-Nielsen et al. 2007, Hyldahl et al. 2010).

Für Aspirin wurden in einer Studie positive, in einer anderen Studie fragliche Behandlungseffekte beobachtet. Riasati et al. ließen 16 Probanden 10 Minuten lang eine Fußbank auf- und absteigen. Direkt nach der Belastung wurde einmalig 200 mg Aspirin (oral) verabreicht. 24 und 48 Stunden nach der Belastung zeigte sich unter der Behandlung mit Aspirin ein signifikant erniedrigter Muskelschmerz im Vergleich zur Placebo-Gruppe (Riasati et al. 2010). Diese Ergebnisse konnten 
Barlas et al. in ihrer Studie nicht bestätigen. Sie beobachteten keinen Behandlungseffekt von Aspirin (Barlas et al. 2000).

Studien zu Diclofenac zeigten sowohl eine positive als auch eine fehlende Wirksamkeit der Behandlung. O'Grady et al. untersuchten 54 Probanden vor- und nachdem sie 20 Minuten eine Fußbank auf- und abgestiegen waren, um dadurch DOMS zu erzeugen. 14 Tage vor der Belastung wurde mit der täglichen Einnahme von $150 \mathrm{mg}$ Diclofenac begonnen, die bis 13 Tage nach der Belastung fortgeführt wurde. Unter der Behandlung mit Diclofenac zeigten sich ein signifikant erniedrigter Muskelschmerz sowie ein beschleunigter Rückgang des Muskelschmerzes (nach 4 Tagen) im Vergleich zu Placebo (nach 7 Tagen) (O'Grady et al. 2000). Donelly et al. beobachten in ihrer Studie wiederum keinen Effekt von Diclofenac auf Muskelschmerz nach exzentrischer Belastung (Donelly et al. 1988).

Für Naproxen wurde in den Studien größtenteils eine positive Wirksamkeit bei Muskelschmerz nach exzentrischer Belastung beschrieben. Dudley et al. erzeugten bei 8 jungen, männlichen Probanden DOMS in der Oberschenkelmuskulatur durch 10 Durchgänge von 7-10 exzentrischen Kontraktionen des M. quadrizeps femoris mit einem Gegengewicht, das 85\% der Maximalkraft entsprach. Direkt nach der Belastung wurde die Einnahme von 220 mg Naproxen dreimal täglich über 10 Tage begonnen. Unter der Behandlung demonstrierten die Probanden reduzierte Muskelschmerzen und -schwellung, sowie eine schnellere Erholung der maximalen Muskelkraft, insbesondere während der ersten 4 Tage nach Belastung (Dudley et al. 1997).

Diese Ergebnisse wurden durch Lecomte et al. bestätigt. Sie ließen in ihrer Studie 20 Probanden ein exzentrisches Belastungsprogramm der Kniestrecker absolvieren. 24 Stunden nach der Belastung wurde mit der Einnahme von 1000 mg Naproxen pro Tag über insgesamt 7 Tage begonnen. Der Muskelschmerz war während seiner maximalen Ausprägung an Tag 3 unter der Behandlung signifikant erniedrigt. Zugleich war die maximale Muskelkraft signifikant erhöht (Lecomte et al. 1998). 
Auch Baldwin et al. zeigten in ihrer Studie ähnliche Ergebnisse. Sie ließen 15 Probanden 8 Durchgänge von 7-10 exzentrischen Kontraktionen des M. quadrizeps femoris mit einem Gegengewicht, das 75\% der Maximalkraft entsprach, absolvieren. Direkt nach der Belastung wurde mit der Einnahme von 220 mg Naproxen dreimal täglich über 10 Tage begonnen. Unter der Behandlung wurde signifikant weniger Muskelschmerz während des Aufstehens vom Stuhl, sowie signifikant weniger Muskelkraftverlust nach 3 Tagen beobachtet. Zudem zeigte sich im MRT ein geringeres Gewebsödem unter Naproxen-Einnahme (Baldwin et al. 2001).

Bourgeois et al. beobachteten im Gegensatz dazu einen negativen Effekt von Naproxen. In der Studie führten 8 Probanden 6 Durchgänge mit jeweils 10 Wiederholungen von exzentrischen Kontraktionen der Kniestrecker bei 85\% der Maximalkraft durch. Vor der Belastung bis 48 Stunden danach wurden 1000 mg Naproxen täglich eingenommen. Die Behandlungsgruppe zeigte eine signifikant verlangsamte Erholung der maximalen Muskelkraft 48 Stunden nach der Belastung im Vergleich zur Referenzgruppe (Bourgeois et al. 1999).

Ketoprofen zeigte in den veröffentlichen Studien einen durchweg positiven Effekt in der Behandlung von Muskelschmerz nach exzentrischer Belastung. Sayers et al. verabreichten 48 Probanden 36 Stunden nach exzentrischer Belastung der Ellenbogenflexoren einmalig entweder 25 mg oder 100 mg Ketoprofen (oral). 8 Stunden nach der Einnahme zeigte sich eine signifikante Muskelschmerzreduktion der 25 mg-Gruppe und ein Trend zur Schmerzreduktion der 100 mg-Gruppe im Vergleich zu Placebo. Beide Gruppen demonstrierten 8 Stunden nach Einnahme eine signifikant fortgeschrittene Erholung der maximalen Muskelkraft im Vergleich zu Placebo (Sayers et al. 2001).

In einer Studie von Cannavino et al. wurde 32 Probanden, beginnend direkt nach der exzentrischen Belastung der Kniestrecker und -beuger, über 2 Tage alle 8 Stunden 100 mg Ketoprofen-Gel (lokal) aufgetragen. Im Vergleich zu Placebo-Gel wurde 24 und 48 Stunden nach Applikation eine signifikante Muskelschmerzreduk- 
tion beobachtet. Hinsichtlich der Erholung der Muskelkraft konnte kein Unterschied festgestellt werden (Cannavino et al. 2003).

Ähnliche Ergebnisse beobachteten Rother et al. in ihren Studien zu Ketoprofen. Sie untersuchten zunächst drei Studien in einer Metaanalyse. 32 Stunden nach der exzentrischen Belastung der Ellenbogenflexoren wurde einmalig entweder 25 mg Ketoprofen oral oder lokal als Ketoprofen-Gel verabreicht und mit Placebo verglichen. 5-12 Stunden nach Applikation zeigte sich eine signifikante Muskelschmerzreduktion unter lokaler Ketoprofen-Anwendung im Vergleich zu Placebo, sowie 12 Stunden nach Applikation im Vergleich zu oral verabreichtem Ketoprofen. Kein Unterschied konnte zwischen der Behandlung mit oralem Ketoprofen und Placebo ausgemacht werden. In einer anschließenden Studie untersuchten Rother et al. den Effekt der 7-tägigen Anwendung von 100 mg Ketoprofen-Gel (lokal). 1 Tag nachdem 20 Probanden in 12 Durchgängen jeweils 120 Treppenstufen herabsteigen mussten (insg. 200 Höhenmeter) wurde mit der Behandlung begonnen. Es konnte unter Ketoprofenanwendung insgesamt eine signifikante Reduktion des Muskelschmerzes im Vergleich zu Placebo beobachtet werden (Rother et al. 2009).

In einer weiteren Studie untersuchten Seidel et al. 24 Probanden nachdem durch Treppenabsteigen DOMS erzeugt wurde. 12 Stunden nach der Belastung wurde mit der Einnahme von 100 mg Ketoprofen (oral) alle 12 Stunden über 7 Tage begonnen. Die Ergebnisse demonstrierten unter der Behandlung mit Ketoprofen eine signifikante Muskelschmerzreduktion innerhalb der ersten 24-36 Stunden nach Applikation. Die Summe des Muskelschmerzes - über den gesamten Nachbeobachtungszeitraum betrachtet - war für die Ketoprofen-Gruppe jedoch signifikant höher. Auch die Erholungszeit bis zum vollständigen Verschwinden des Muskelschmerzes war unter Ketoprofen-Einnahme im Vergleich zu Placebo signifikant verlängert (Seidel et al. 2012).

Positive Effekte von Celecoxib auf Muskelschmerz nach exzentrischer Belastung wurden in mehreren Studien demonstriert. Paulsen et al. begannen mit der 9- 
tägigen Gabe von täglich $400 \mathrm{mg}$ Celecoxib (oral) 45 Minuten vor der exzentrischen Belastung der Ellenbogenflexoren bei 33 Probanden. Eine Muskelschmerzreduktion unter Celecoxib-Einnahme wurde beobachtet. Hinsichtlich der Erholung der maximalen Muskelkraft konnte im Vergleich zu Placebo kein Unterschied festgestellt werden (Paulsen et al. 2010).

Die Ergebnisse wurden durch eine Studie von Rother et al. bestätigt. 80 Probanden absolvierten ein exzentrisches Belastungsprogramm, bestehend aus Absteigen von Treppenstufen mit insgesamt 460-600 Höhenmetern. Direkt nach der Belastung wurde mit der Gabe von 200 mg Celecoxib (oral) alle 12 Stunden über den Zeitraum von 60 Stunden begonnen. Im Vergleich zu Placebo zeigte sich unter der Behandlung eine moderate Schmerzreduktion. Ein Unterschied hinsichtlich der Erholung der maximalen Muskelkraft wurde nicht beobachtet (Rother et al. 2011).

Es wurden bislang keine Studien durchgeführt, in denen die Wirksamkeit von Etoricoxib in der Behandlung von DOMS untersucht wurde. In Kapitel 5.3 werden die Ergebnisse aller o. g. Studien noch einmal detailliert und in Bezug auf die Ergebnisse der Etoricoxib-Studie bewertet. Verschiedene Faktoren, die in den Studien möglicherweise Einfluss auf die Ergebnisse genommen und eine unterschiedliche Bewertung der Wirksamkeit einer Therapie bewirkt haben könnten, werden gegenübergestellt und diskutiert (siehe Tab. 27). 


\section{ZIELE DER ARBEIT}

\subsection{Hauptziel}

Hauptziel der Arbeit ist es, die Wirksamkeit antientzündlicher Therapie (Etoricoxib) auf Muskelschmerz nach exzentrischer Belastung zu untersuchen, um Rückschlüsse auf die Beteiligung der Entzündungsreaktion an DOMS ziehen zu können.

Die Ergebnisse sollen in Bezug auf vergleichbare klinische Studien, bei denen die Wirksamkeit anderer NSAR auf DOMS untersucht wurde, diskutiert werden. Dabei werden in erster Linie folgende, wesentliche Faktoren verglichen: die unterschiedlich starke antientzündliche Potenz der verschiedenen NSAR, der Zeitpunkt der ersten Medikamentenapplikation nach exzentrischer Belastung und die Häufigkeit der Medikamenteneinnahme im Untersuchungszeitraum.

Unter Berücksichtigung oben genannter Einflussfaktoren sollen Aussagen zur Bedeutung der Entzündungsreaktion auf die Entwicklung und den Verlauf des Muskelschmerzes und der Regeneration des Muskels nach exzentrischer Belastung ermöglicht werden.

\section{$2.2 \quad$ Nebenziele}

Weiterhin soll der Einfluss des Alters auf die Entwicklung und die Ausprägung der Entzündungsreaktion bei DOMS untersucht werden. Unterschiede bei der Entwicklung des Schmerzes und der Erholungsfähigkeit der Muskulatur nach exzentrischer Belastung zwischen jüngeren und älteren Erwachsenen sind zu prüfen. Auch die antientzündliche Therapie könnte in beiden Altersgruppen aufgrund altersbedingt unterschiedlicher Reparaturvorgänge differenzierte Effekte auf DOMS haben.

Zuletzt ist hinsichtlich der Sicherheit und Verträglichkeit zu evaluieren, ob eine Anwendung von NSAR zur Therapie von Muskelschmerz nach exzentrischer Belastung zu empfehlen ist. 


\subsection{Arbeitshypothesen}

$\mathrm{H}_{1}$ : Es gibt keinen Unterschied zwischen der Wirksamkeit von Etoricoxib und Placebo auf Muskelschmerz, auf die Erholung der Muskelkraft und auf Entzündungsmarker im Blut nach exzentrischer Belastung.

$\mathrm{H}_{2}$ : Die unterschiedlich starke antientzündliche Potenz der NSAR, der Zeitpunkt der ersten NSAR-Einnahme nach exzentrischer Belastung und die Häufigkeit der NSAR-Gabe haben keinen Einfluss auf den zeitlichen Verlauf von DOMS.

$\mathrm{H}_{3}$ : Es gibt hinsichtlich des Alters keinen Unterschied zwischen der Wirksamkeit von Etoricoxib und Placebo auf Muskelschmerz, auf die Erholung der Muskelkraft und auf Entzündungsmarker im Blut nach exzentrischer Belastung.

$\mathrm{H}_{4}$ : NSAR sollten nicht bei Muskelschmerz nach exzentrischer Belastung angewandt werden. 


\section{METHODIK}

\subsection{Studiendesign}

Es handelt sich um eine prospektive, randomisierte, doppelblinde (probanden- und untersucherverblindete), placebo-kontrollierte, crossover-Studie zur Beurteilung von Etoricoxib für die Behandlung von Muskelschmerz und Entzündung nach exzentrischer Belastung.

Die Probanden mussten in der Studie zwei Phasen durchlaufen. Die Untersuchung beschränkte sich in einer Phase jeweils auf nur ein Bein. Welches Bein in Phase 1 und welches in Phase 2 untersucht wurde, wurde randomisiert festgelegt. Zu Beginn von Phase 1 erfolgte die exzentrische Belastung der Oberschenkelmuskulatur auf dem Trainingsgerät „extra fit Beinstrecker”, um DOMS bei den Probanden zu erzeugen. Nach $16 \pm 2$ Stunden wurde evaluiert, ob ein ausreichender Muskelschmerz induziert werden konnte und nach der randomisierten Zuteilung zu einer Behandlungsgruppe die Behandlung mit der Prüfmedikation (Etoricoxib) oder der Vergleichsmedikation (Placebo) begonnen. Die jeweilige Medikation wurde ein mal täglich für 7 Tage im 24-Stundenintervall eingenommen. Vor der täglichen Medikamenteneinnahme wurde die Auswirkung der exzentrischen Belastung auf den Muskelschmerz im Ruhezustand („Ruheschmerz“), auf den Muskelschmerz während konzentrischer Muskelkontraktionen („Bewegungsschmerz“), auf die Wiederherstellung der maximalen konzentrischen Muskelkraft („Maximalkraft“), auf die Schmerzschwelle am druckempfindlichsten Punkt des Muskelkaters (,maximaler Druckpunkt") sowie auf die Entzündungsmarker im Blut untersucht. Nach einer Auswaschphase von mindestens 5 Tagen führten die Probanden in Phase 2 der Studie die exzentrische Belastung mit dem kontralateralen Bein aus. Probanden, die in Phase 1 Etoricoxib erhalten hatten, erhielten in Phase 2 Placebo und umgekehrt. Alle Probanden unterzogen sich den gleichen Untersuchungsmethoden wie in Phase 1. Ein Schema des Studiendesigns ist in Abb. 1 dargestellt. 


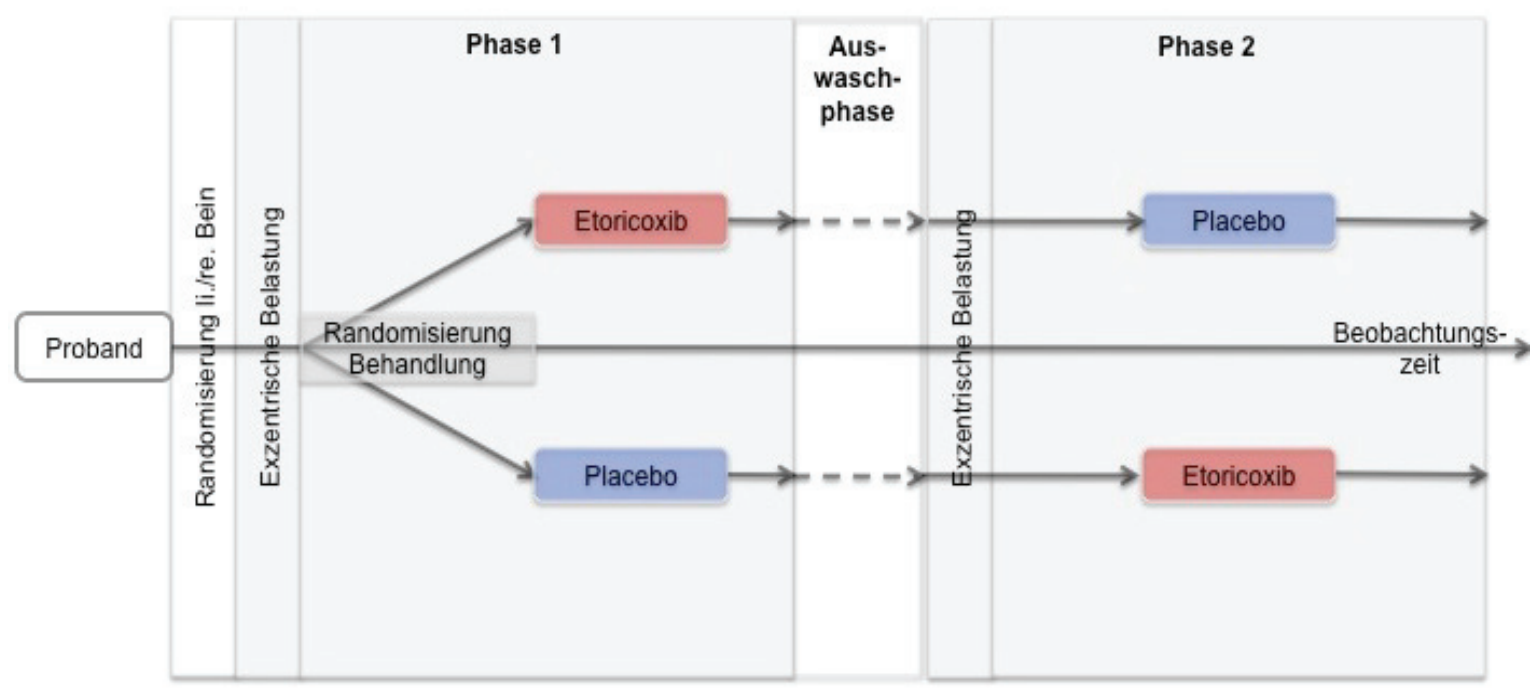

Abb. 1: Schema des crossover-Designs der Studie (nach Wellek und Blettner 2012)

Die Studie (Studiennummer: XPM-032) wurde in den Untersuchungsräumen der Fa. X-pertMed in Jena in enger Zusammenarbeit mit dem Zentrum für Physikalische und Rehabilitative Medizin des Sophien- und Hufeland-Klinikums Weimar durchgeführt.

Die Ethikkommission der Landesärztekammer Thüringen erteilte am 14. Dezember 2010 die Zustimmung zur Durchführung der Studie (siehe Anh. 1).

\subsection{Auswahl der Probanden}

Es wurden insgesamt 50 gesunde, freiwillige Erwachsene in zwei verschiedenen Altersklassen gesucht: 25 jüngere Erwachsene zwischen 18 und 40 Jahren sowie 25 ältere Erwachsene zwischen 50 und 70 Jahren. In Tab. 1 und 2 sind die Einund Ausschlusskriterien aufgelistet, die zur Teilnahme an der Studie von den Probanden erfüllt werden mussten, bzw. nicht vorliegen durften. 
- unterschriebene und datierte Einverständniserklärung vor Teilnahme

- gesunde Freiwillige, wie durch den Prüfarzt festgelegt

- Alter 18-40 oder 50-70 Jahre

- $\mathrm{BMI}>20$ und $<30$

- Bewegungsschmerz im Testmuskel (Oberschenkel) von mindestens 5 auf einer 11stufigen numerischen Rating Skala (NRS)

- Bereitschaft, auf jegliche physikalische Therapie, harte körperliche Arbeit, Belastungen und Sauna für die Dauer der Studie zu verzichten

- Frauen im gebärfähigen Alter müssen eine verlässliche Verhütungsmethode anwenden (eine Methode mit weniger als 1\% Fehlerquote, z. B. Implantate, Injektionen, einige Intrauterinpessare, sexuelle Enthaltsamkeit, ein vasektomierter Partner)

Tab. 2: Ausschlusskriterien

- Teilnahme an einer anderen klinischen Studie während der letzten 30 Tage und für die gesamte Dauer dieser Studie

- Insassen eines psychiatrischen Krankenhauses, Gefängnisses oder einer anderen staatlichen Institution

- Prüfarzt oder anderes Studienpersonal, das direkt oder indirekt an der Durchführung dieser klinischen Studie beteiligt ist

- Schwangerschaft oder Stillperiode

- Alkohol- oder Drogenmissbrauch

- Maligne Erkrankungen in den letzten zwei Jahren

- bekannte Hypersensitivität auf Etoricoxib oder andere NSAR einschließlich COX-2 Hemmer bzw. deren Zusatzstoffe

- Bronchialspasmus, akute Rhinitis, angioneurotisches Ödem, Urtikaria oder allergieartige Reaktionen auf NSAR einschließlich COX-2 Hemmer

- größere Traumata (z. B. Brüche, Sehnen- oder Muskelrisse) des Bewegungsapparates der unteren Extremität

- Schmerzzustände, welche die Schmerzbeurteilung während der Studie beeinflussen können (z. B. neuropathischer Schmerz)

- neurologische oder psychiatrische Symptome, welche zu Desorientierung, Gedächtnisstörungen oder der Unfähigkeit sorgfältig zu berichten führen (z. B. Depressive Störungen, Alzheimer, Schizophrenie oder andere Psychosen) 
- Herzinsuffizienz (NYHA II-IV)

- Hypertonie mit anhaltendem Blutdruck $>140 / 90 \mathrm{mmHg}$ und nicht ausreichend eingestellt

- klinisch gesicherte koronare Herzkrankheit, periphere arterielle Verschlusskrankheit und/oder zerebrovaskuläre Erkrankungen

- Schlaganfall oder Herzinfarkt in der Anamnese

- klinisch relevante EKG-Veränderungen

- geschätzte Kreatinin Clearance $<60 \mathrm{ml} / \mathrm{min}$

- Leberfunktionsstörung (z. B. definiert durch ALAT und/oder ASAT Werte über dem oberen Normwert)

- Blutgerinnungsstörungen oder Blutungsneigung, oder Einnahme von Antikoagulantien inklusive niedrig dosiertem Aspirin

- Pankreatitis, peptische Ulzera oder gastrointestinal Blutung in der Anamnese

- entzündliche Magen-Darmerkrankungen (z. B. Morbus Crohn, Colitis Ulcerosa)

- jegliche Analgetikabehandlung einschließlich Husten- und Erkältungsmittel, welche schmerzstillende Eigenschaften haben

- jegliche Behandlung mit Medikamenten welche die Schmerzempfindung beeinflussen, wie z. B. ZNS wirksame Medikamente

- Einnahme von Statinen innerhalb der letzten drei Monate seit Screening und während der Studie

- Einnahme von oralen Kontrazeptiva

\subsection{Studienablauf}

Die Probanden wurden zu mehreren „Visiten“ einbestellt, in denen Screening, exzentrische Belastung, sowie regelmäßige Nachbeobachtungen stattfanden (siehe Tab. 3). 


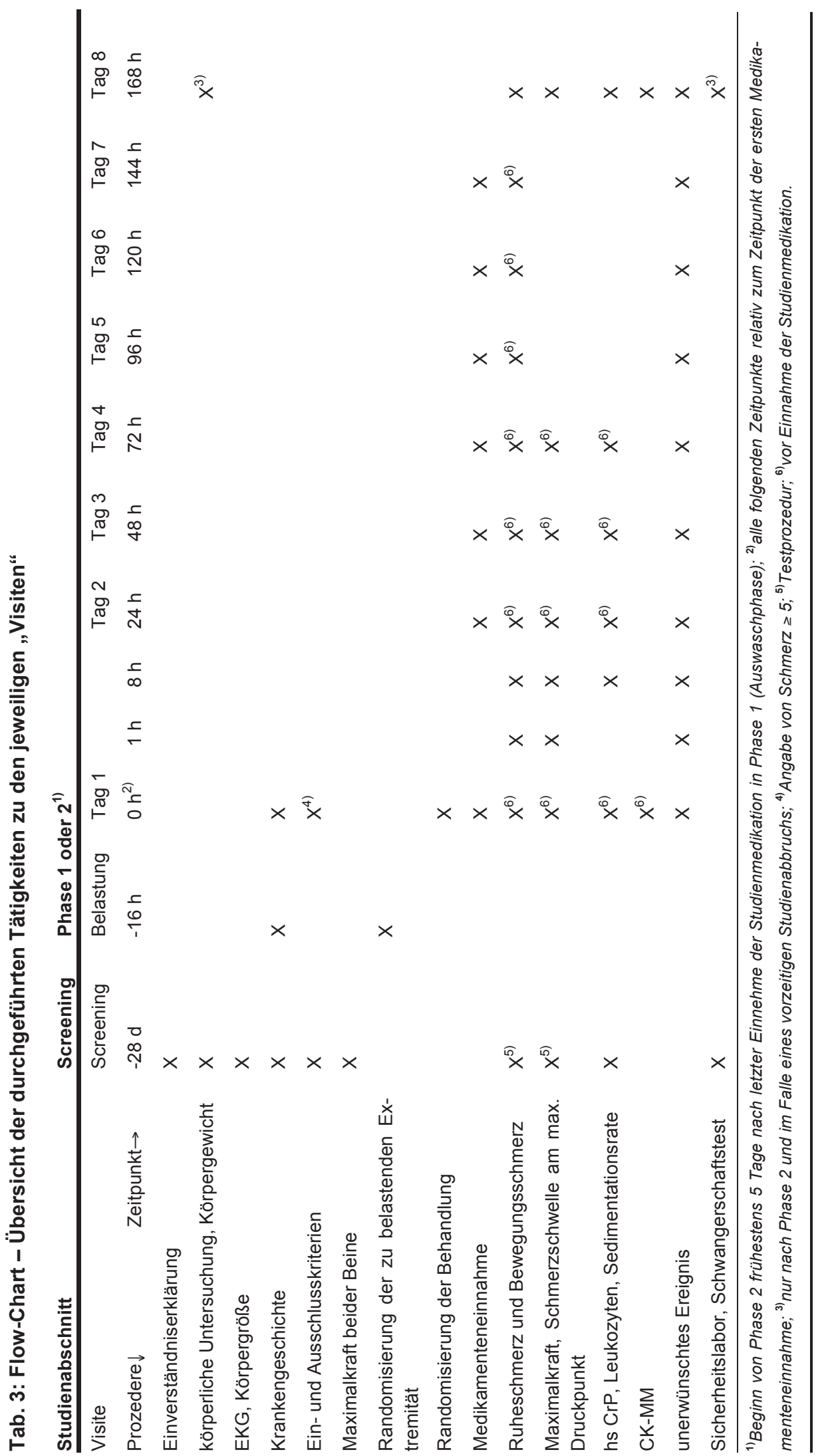




\subsubsection{Screening}

Das Screening diente dazu, Ein- und Ausschlusskriterien zu prüfen sowie den Probanden eine Einführung in die Studienprozeduren zu geben.

Zunächst wurden Informationen über die Studie bereitgestellt, mit denen sich die Probanden ausführlich beschäftigen konnten. Ziele und Ablauf der Studie wurden detailliert erklärt. Die Probanden hatten jederzeit die Möglichkeit, dem Studienpersonal und dem Prüfarzt weitere Fragen zu stellen. Daraufhin war eine Einverständniserklärung zu unterzeichnen.

Jedem zum Screening einbestellten Probanden wurde eine Screeningnummer zugeteilt. Diese bestand aus einem "S“ und einer fortlaufenden dreistelligen Nummer, die den Probanden in aufsteigender Reihenfolge zugeteilt wurde (z. B. S001, S002, S003 etc.). Anhand der individuellen Screeningnummer und der Studiennummer (XPM-032) war jeder einzelne Proband, der am Screening teilgenommen hatte, identifizierbar.

Nach Aufnahme der demographischen Daten und der persönlichen medizinischen Vorgeschichte wurde durch den Prüfarzt eine körperliche Untersuchung durchgeführt. Diese beinhaltete die Bewertung von Allgemeinzustand, Haut, Hals, Augen, Ohren, Nase, Rachen, Lungen, Herz, Abdomen, Rücken, Nervensystem, unterer Extremität und 12-Kanal EKG. Vitalparameter (Puls, Blutdruck), Körpergewicht und Körpergröße wurden durch das Studienpersonal erhoben.

Eine Blutprobe zur Bestimmung folgender Parameter wurde entnommen: Differential Blutbild (Hämoglobin, Hämatokrit, Blutplättchen), AlaninAminotransferase (ALAT), Aspartat-Aminotransferase (ASAT), Gesamtes Bilirubin, Alkalische Phosphatase, Harnstoff, Glomeruläre Filtrationsrate (GFR), Serum Albumin, Partielle Thromboplastinzeit (PTT), Thromboplastinzeit (Quick), hs CrP, Sedimentationsrate und Leukozyten. Bei Frauen in potenziell gebärfähigem Alter wurde zusätzlich ein Urin-Schwangerschaftstest durchgeführt. 
Es folgte die Einführung der Probanden in die Studien- und Testprozeduren: Zur Evaluierung des Ruheschmerzes und des Bewegungsschmerzes wurde eine 11-stufige numerische Rating Skala (NRS) verwendet. Auf der numerischen Skala von 0-10 war die Schmerzintensität einzuordnen. Die Zahl 0 bedeutet, dass „kein Schmerz" vorhanden ist, die Zahl 10 beschreibt die „stärksten vorstellbaren Schmerzen“. Zusätzlich konnte die NRS in Kombination mit der Wong-Baker-Faces-Schmerzskala verwendet werden, bei der die verschiedenen Schmerzintensitäten als Piktogramm („Smileys“) visualisiert sind. Zum korrekten Verständnis und zur sicheren Anwendung der Schmerzskalen wurde während des Screenings das Bewerten von Ruheschmerz und von Bewegungsschmerz mit den Probanden erprobt. Zur Bewertung des Ruheschmerzes mussten die Probanden in ruhiger, sitzender Körperposition ihre dabei subjektiv empfundenen Schmerzen im Oberschenkel auf der NRS einschätzen. Die Bewertung des Bewegungsschmerzes mussten die Probanden während der Bestimmung der Maximalkraft wie folgt abgeben: Nachdem die Probanden die konzentrischen Kontraktionen der Oberschenkelmuskulatur zur Bestimmung der aktuellen Maximalkraft durchgeführt hatten, mussten sie ihre dabei subjektiv empfundenen Schmerzen im Oberschenkel auf der NRS einschätzen.

Weiterhin wurde das Prozedere der Schmerzschwellenbestimmung am max. Druckpunkt des Muskelkaters vorgestellt und geübt. Die Schmerzschwelle wurde durch Hitzestimulation ermittelt. Am Übergang des M. rectus femoris in seine Ansatzsehne wurde der auf Palpation empfindlichste Punkt im Muskel bestimmt. Durch eine Thermode ( $9 \times 9 \mathrm{~mm}$, Somedic AB, Stockholm, Schweden) wurden Hitzestimuli auf diesen max. Druckpunkt appliziert. Die Thermode wurde durch ein an ihrer Oberfläche befestigtes „Thermolement“ über einen Feedbackmechanismus reguliert. Um die Gefahr von Hautschäden auszuschließen, wurde die maximale Hitzeapplikation der Thermode auf $52^{\circ} \mathrm{C}$ begrenzt. Die Temperatur der Thermode wurde ausgehend von einer Anpassungstemperatur von $32^{\circ} \mathrm{C}$ um $1^{\circ} \mathrm{C}$ pro Sekunde langsam erhöht. Die Probanden wurden angewiesen einen Knopf zu drücken, sobald sie die Hitze der Thermode als 
schmerzhaft empfinden würden, um die Hitzezufuhr zu beenden. Auf Knopfdruck wurde die Temperatur der Thermode sofort um $3^{\circ} \mathrm{C}$ pro Sekunde bis zur Ausgangstemperatur $\left(32^{\circ} \mathrm{C}\right)$ herabgesetzt. Die Temperaturen an der erreichten Schmerzschwelle wurden durch das Thermoelement gemessen und elektronisch aufgezeichnet. Es wurde jeweils eine Serie von fünf aufeinanderfolgenden Messungen durchgeführt. Der Mittelwert der letzten drei Messungen repräsentiert die Schmerzschwelle am max. Druckpunkt (siehe Anh. 4).

Daraufhin wurden die Probanden in das Trainingsgerät „extra fit Beinstrecker" (Extrafit Investments $\mathrm{GmbH}$, Deutschland) eingewiesen (siehe Anh. 2). Die Stuhllehne wurde für jeden Probanden individuell eingestellt, so dass eine optimale Sitzposition mit ca. $90^{\circ}$ Beugewinkel in Hüft- und Kniegelenken erreicht wurde. Die Beinrolle wurde so positioniert, dass sie auf dem distalen Drittel des ventralen Unterschenkels des zu belastenden Beins lastete und hierüber das Gewicht bewegt wurde. Das andere Bein wurde in bequemer Position neben der Beinrolle vom Stuhl herabgehangen.

Nun wurde nacheinander die Maximalkraft (maximale konzentrische Kraftaufwendung) für jeweils das linke und das rechte Bein bestimmt. Zur Maximalkraftbestimmung wurden konzentrische Kontraktionen der Oberschenkelmuskulatur durchgeführt. Dabei wurden direkt aufeinanderfolgend, bis zur absoluten Erschöpfung Kniestreckbewegungen gegen ein individuell festgelegtes Gewicht (50\% des Körpergewichts) durchgeführt. Die Anzahl der erreichten Kniestreckungen wurde aufgezeichnet. Insgesamt absolvierten die Probanden hiervon unter maximaler Kraftanstrengung drei Serien. Der Mittelwert der erreichten Anzahl an Kniestreckungen repräsentiert den Wert der Maximalkraft.

Abschließend absolvierten die Probanden zur Einübung des Bewegungsablaufes eine exzentrische Probebelastung ohne Gegengewicht. Ein Termin zur richtigen exzentrischen Belastung wurde innerhalb der nächsten 28 Tage angesetzt. 


\subsubsection{Exzentrische Belastung}

Die exzentrische Belastung diente dazu, bei den Probanden in einer standardisierten Form DOMS zu erzeugen.

Zunächst wurde jedem Probanden durch einen nicht verblindeten Mitarbeiter der Studie mit Hilfe einer zuvor vorbereiteten Liste randomisiert zugeteilt, welches Bein in Phase 1 Ziel der exzentrischen Belastung sein sollte. Das andere Bein war demzufolge in Phase 2 zu belasten. Die Randomisierungsliste sah vor, dass die Hälfte der Probanden die Belastung mit dem rechten Bein in Phase 1 begannen und die andere Hälfte mit dem linken Bein. Führte der erste Proband die exzentrische Belastung zunächst mit dem rechten Bein durch, musste der als nächstes folgende Proband die Belastung mit dem linken Bein, der darauf folgende Proband wieder mit dem rechten Bein beginnen. In dieser Form wurde jeder Proband vor der Belastung auf der Liste fortführend erfasst und die Reihenfolge für die folgenden Probanden entsprechend angewandt.

Vor der Belastung wurden Veränderungen der Begleitmedikation oder in der medizinischen Vorgeschichte seit der letzten Visite (Screening) überprüft. Die exzentrische Belastung am Trainingsgerät „extra fit Beinstrecker" wurde wie folgt durchgeführt: Die Probanden saßen in der individuell voreingestellten Position auf dem Trainingsstuhl. Das Knie des zu belastenden Beins wurde gegen einen Widerstand von 5-10 kg langsam bis auf ca. $0^{\circ}$ gestreckt, kurz in Streckung gehalten und langsam wieder in die ursprüngliche Position von $90^{\circ} \mathrm{Beu}-$ gung zurückgeführt. Dieser Rückführbewegung wurde durch das Trainingsgerät ein individuell berechnetes Gewicht (45\% der für das entsprechende Bein während des Screenings ermittelten Maximalkraft) entgegengesetzt. Demzufolge mussten die Probanden einer schnellen Beugung im Kniegelenk durch das Gegengewicht während der Rückführbewegung entgegenarbeiten (siehe Anh. 3). Auf diese Art und Weise wurde eine erhebliche exzentrische Belastung der Oberschenkelmuskulatur (M. quadrizeps femoris) erreicht. Ein Bewegungszyklus (entsprechend: $90^{\circ}$ Beugung $-0^{\circ}$ Streckung $-90^{\circ}$ Beugung) musste über 
einen Zeitraum von genau 4 Sekunden durchgeführt werden. Die Bewegungszyklen wurden solange wiederholt, bis das Gewicht nicht mehr innerhalb des 4Sekundenzeitraums zurückbewegt werden konnte - definiert als "gewollte Erschöpfung“. Nach einer Minute Pause wurde ein weiterer Durchgang bis zur wiederholten Erschöpfung durchlaufen. Insgesamt wurden drei Durchgänge absolviert.

\subsubsection{Nachbeobachtungsphase}

\section{Tag: $0 h$}

Die erste Visite der Nachbeobachtungsphase wurde $16 \pm 2$ Stunden nach exzentrischer Belastung durchgeführt.

Zunächst wurde überprüft, ob ausreichend Muskelschmerz nach exzentrischer Belastung erzeugt wurde. Dazu wurde die aktuelle Maximalkraft des belasteten Beins bestimmt und die Probanden zu ihrem Schmerz während dieser konzentrischen Kontraktionen (Bewegungsschmerz) befragt. Lediglich solche, die während der Belastung einen Schmerz im Oberschenkel von $\geq 5$ auf der NRS angaben und damit auch dieses Einschlusskriterium erfüllten, waren geeignet an der Studie teilzunehmen.

Neben der Maximalkraft und des Bewegungsschmerzes wurden der Ruheschmerz, die Schmerzschwelle am max. Druckpunkt, sowie die Entzündungsmarker (hs CrP, Sedimentationsrate, Leukozyten) im Blut während dieser Visite bestimmt.

Die Probanden wurden nun innerhalb der jeweiligen Altersgruppen in einem Verhältnis von 1:1 zu einer der folgenden Behandlungsgruppen für Phase 1 der Studie randomisiert zugeteilt:

1. Arcoxia $^{\circledR}$ (Prüfmedikation)

Arcoxia gehört als schmerz- und entzündungshemmendes Medikament zu der Klasse der nichtsteroidalen Antirheumatika (NSAR). Etoricoxib, der Wirkstoff 
von Arcoxia", ist ein hochselektiver Hemmstoff des Enzyms Cyclooxygenase-2 (COX-2). Die COX-2 beschleunigt die Synthese von Prostaglandinen, die für Schmerz und Entzündung verantwortlich sind, indem es die Oxidation von Arachidonsäure zu Prostaglandinen katalysiert (Dallob et al. 2003). Das Enzym wird vermutlich durch proinflammatorische Zytokine induziert und ist vermehrt in entzündetem Gewebe vorhanden (Furst 1999). Durch die im Vergleich zu nichtselektiven NSAR nur geringe Hemmung des Enzyms Cyclooxygenase-1 (COX1), das konstitutiv im Gewebe exprimiert wird und durch die Synthesehemmung zum einen von Prostaglandinen der Magenschleimhaut in die Magenschleimproduktion und zum anderen von Thromboxan $B_{2}$ in die Hämostase eingreift, ergibt sich für Arcoxia ein entsprechend abweichendes Nebenwirkungsprofil (Wallace 1999). Als Nebenwirkungen werden u.a. Ödeme, Hypertonie, Palpitationen, Schwindel und Kopfschmerzen, GIT-Beschwerden, Ekchymose sowie Müdigkeit beschrieben. Arcoxia* ist in Deutschland seit September 2004 für Personen ab 16 Jahren zur Behandlung von Arthrose, rheumatoider Arthritis, akuter Gichtarthritis und Morbus Bechterew zugelassen. Die orale Bioverfügbarkeit beträgt ca. 100\%. Es wird rasch absorbiert und führt zu einem schnellen Wirkeintritt mit einer maximalen Plasmakonzentration ca. eine Stunde nach Einnahme. Bei einer Halbwertszeit von ca. 22 Stunden und einer Wirkdauer von 24 Stunden wird Arcoxia ein mal täglich eingenommen (MSD 2011). Neben dem Wirkstoff enthält Arcoxia folgende Trägerstoffe: Calciumhydrogenphosphat, Croscarmellose-Natrium, Magnesiumstearat, mikrokristalline Cellulose, Carnaubawachs, Lactose-Monohydrat, Hypromellose, Titandioxid (E 171), Triacetin. Arcoxia' wird zur Behandlung von Symptomen bei Reizzuständen degenerativer und entzündlicher Gelenkerkrankungen (Arthorse und rheumatoider Arthritis) sowie von Schmerzen und Entzündungszeichen bei akuter Gichtarthritis eingesetzt. Die tägliche Maximaldosis von Arcoxia* beträgt 120 mg. Diese Dosierung ist lediglich zur Behandlung von Gichtanfällen zugelassen. Somit wurde als Studiendosis die nächst niedrigere Dosierung festgelegt: eine 90 mg Filmtablette pro Tag, oral dargereicht für 7 Tage, im 24-Stundenintervall. 
Die erste Medikamentengabe erfolgte $16 \pm 2$ Stunden nach exzentrischer Belastung.

\section{Placebo (Vergleichsmedikation)}

Um die Wirksamkeit von Etoricoxib zu prüfen, wurde in der anderen Behandlungsgruppe zum Vergleich ein Placebo ohne Wirkstoff verabreicht. Es wurden $8 \mathrm{~mm}$ große, weiße P-Tabletten der Fa. Lichtenstein verwendet. Als Trägerstoffe sind Cellulosepulver, Lactose-Monohydrat, Magnesiumstearat, mikrokristalline Cellulose enthalten. Darreichungsform und -modus entsprachen denen der Arcoxia-Gruppe: eine Tablette pro Tag, oral dargereicht für 7 Tage, im 24 Stundenintervall. Die erste Medikamentengabe erfolgte $16 \pm 2$ Stunden nach exzentrischer Belastung.

Die Zuteilung der Probanden in die jeweilige Behandlungsgruppe erfolgte mit Hilfe der Methode der verborgenen Zuordnung („concealed allocation“). Hierfür wurden versiegelte Umschläge verwendet. Ein nicht verblindeter Mitarbeiter der Studie öffnete den für die Screeningnummer des entsprechenden Probanden vorgesehenen Umschlag. Dieser enthielt eine Liste, auf der die Anwendung der jeweiligen Behandlungsmedikation in Phase 1 und Phase 2 für den Probanden festgelegt war. Zu jeder Zuteilungsliste gehörte eine Behandlungsnummer, die durch ein „T“ und einer fortlaufenden zweistelligen Nummer gekennzeichnet war (z. B. T01, T02, T03 etc.) und die jedem entsprechend randomisierten Probanden zugeordnet wurde. Auf diese Weise wurde die Zuordnung des Probanden zur entsprechenden Behandlungsgruppe gekennzeichnet, auch für den Fall einer nötigen Entblindung.

Die entsprechende Studienmedikation wurde daraufhin durch das nicht verblindete Studienmitglied verabreicht. Die Probanden trugen zum Zwecke der Verblindung während der Medikamenteneinnahme eine Augenbinde. Das nicht verblindete Studienpersonal behandelte jegliche Informationen bezüglich der Studienmedikation vertraulich und war nicht an der Auswertung erhobener Probandendaten beteiligt, die durch das Wissen um die Zuteilung der Studienmedi- 
kation hätten verzerrt werden können. Das Studienpersonal, das an der Ergebnisauswertung beteiligt war, blieb verblindet und nahm an der Versuchsdurchführung nicht teil.

Der Zeitpunkt der ersten Medikamenteneinnahme wurde als "Zeitpunkt $0 \mathrm{~h}$ “ festgelegt. Alle folgenden Visitenzeiten beziehen sich auf diesen Zeitpunkt.

\section{Tag: $1 \mathrm{~h}$}

Die Visite wurde 1 Stunde \pm 20 min nach der ersten Medikamenteneinnahme durchgeführt. Ruheschmerz, Schmerzschwelle am max. Druckpunkt, Maximalkraft und Bewegungsschmerz wurden bestimmt.

\section{Tag: $8 h$}

Die Visite wurde $8 \pm 1$ Stunden nach der ersten Medikamenteneinnahme durchgeführt. Ruheschmerz, Schmerzschwelle am max. Druckpunkt, Maximalkraft und Bewegungsschmerz wurden bestimmt, sowie eine Blutentnahme zur Bestimmung der Entzündungsmarker (hs $\mathrm{CrP}$, Sedimentationsrate, Leukozyten) durchgeführt.

\section{2.-4. $\mathrm{Tag}$}

Die Visiten wurden $24 \pm 2$ Stunden, $48 \pm 3$ Stunden bzw. $72 \pm 4$ Stunden nach der ersten Medikamenteneinnahme durchgeführt. Ruheschmerz, Schmerzschwelle am max. Druckpunkt, Maximalkraft und Bewegungsschmerz wurden jedesmal bestimmt, sowie Blutentnahmen zur Bestimmung der Entzündungsmarker (hs CrP, Sedimentationsrate, Leukozyten) durchgeführt. Danach wurde die tägliche Studienmedikation verabreicht.

\section{5.-7. Tag}

Die Visiten wurden $96 \pm 5$ Stunden, $120 \pm 6$ Stunden bzw. $144 \pm 6$ Stunden nach der ersten Medikamenteneinnahme durchgeführt. Ruheschmerz und Bewegungsschmerz wurden bestimmt. Danach wurde die tägliche Studienmedikation verabreicht. 


\section{Tag}

Die Visite wurde $168 \pm 6$ Stunden nach der ersten Medikamenteneinnahme durchgeführt. Ruheschmerz, Schmerzschwelle am max. Druckpunkt, Maximalkraft und Bewegungsschmerz wurden bestimmt sowie eine Blutentnahme zur Bestimmung der Entzündungsmarker (hs CrP, Sedimentationsrate, Leukozyten) durchgeführt.

Falls dies die letzte Visite in Phase 1 war, wurde ein Termin für die exzentrische Belastung von Phase 2 festgelegt. Phase 2 der Studie durfte erst nach einer Auswaschphase von mindestens 5 Tagen beginnen, um zu gewährleisten, dass die Wirkung des ersten Medikamentes vollständig abgebaut war und keine Übertragungseffekte („,carry-over effects") in Phase 2 der Studie auftraten.

Falls dies die letzte Visite in Phase 2 war, wurde eine abschließende körperliche Untersuchung durch den Prüfarzt durchgeführt. Klinisch relevante Veränderungen im Vergleich zur körperlichen Voruntersuchung während des Screenings mussten als unerwünschtes Ereignis (AE) gemeldet werden. Zusätzlich wurde im Blut ein „Sicherheitslabor" mitbestimmt und bei Frauen in potenziell gebärfähigem Alter ein Schwangerschaftstest durchgeführt.

\subsection{Studienpopulation}

Alle Probanden, die mindestens einmal die Studienmedikation eingenommen hatten und eine nachfolgende Visite absolvierten, wurden in die „safety Population" eingeschlossen. Zur Auswertung der Wirksamkeitskriterien wurde die "intention-to-treat Population“ (ITT) herangezogen (,full set analysis“). Diese Population beinhaltet alle Probanden, die randomisiert wurden und mindestens einmal die Studienmedikation eingenommen hatten. Die „per protocol Population" (PP) besteht aus jenen Probanden der ITT-Population, die keine Abweichungen vom Studienprotokoll vorwiesen.

Insgesamt wurden 65 gesunde Erwachsene in das Screeningverfahren für die Studie einbezogen. 15 der gescreenten Probanden wurden nicht in die Rando- 
misierung eingeschlossen. Grund dafür war eine unzureichende Erzeugung von DOMS nach exzentrischer Belastung (Bewegungsschmerz < 5) bei 14 Probanden. Ein Proband widerrief seine Einverständniserklärung aus persönlichen Gründen.

50 Probanden wurden in die Studie eingeschlossen und erhielten mindestens einmal die Studienmedikation. Von diesen vollendeten 47 Probanden (94\%) die Studie, drei Probanden (6\%) mussten vorzeitig abbrechen. Zwei Abbrüche hatten administrative Gründe, d. h. ein unzureichendes Verständnis des Studienablaufes seitens der Probanden. Ein Proband konnte die Studie aufgrund eines schweren unerwünschten Ereignisses („severe adverse event“ - SAE), das nicht durch die Studienmedikation bedingt war, nicht vollenden.

In unserer Studie war die ITT-Population mit der PP-Population identisch. Sie bestand aus insgesamt 48 Probanden. Davon waren 23 Frauen und 25 Männer. 23 Probanden zählten zu der Gruppe der jüngeren Erwachsenen im Alter zwischen 18 und 40 Jahren (Mittelwert: 29,6, Median: 28). 25 Probanden zählten zu der Gruppe der älteren Erwachsenen im Alter zwischen 50 und 70 Jahren (Mittelwert: 59,9, Median: 60).

\subsection{Statistische Methoden}

Die Bewertung der belastungsbezogenen Veränderungen der Kriterien Ruheschmerz und Maximalkraft erfolgt durch den Vergleich von Ausgangswerten mit Werten nach exzentrischer Belastung. Hierzu wurde der „Zweistichproben-tTest" herangezogen. Dieser Signifikanztest ermöglicht Aussagen dazu, ob Mittelwerte zweier Gruppen gleich sind oder sich voneinander unterscheiden.

Die konfirmatorische Auswertung zur Bewertung der Wirksamkeit der Behandlung prüft die Behandlungsgruppen Etoricoxib und Placebo hinsichtlich der Kriterien Ruheschmerz, Bewegungsschmerz, Schmerzschwelle am max. Druckpunkt, Maximalkraft und Entzündungsmarker. Bei dem crossover-Design der Studie diente jeder Proband als seine eigene Kontrolle. Die Probanden wurden 
für die Auswertung zufällig in zwei Sequenzgruppen (A-B und B-A) eingeteilt. Es wurden Vergleiche zwischen diesen beiden Gruppen aufgestellt. Die intraindividuellen Differenzen zwischen den Daten, die jeweils in den beiden Studienphasen erhoben wurden, waren dabei entscheidend. Um eine statistisch gültige Beurteilung der Behandlungseffekte machen zu können, wurde der zweiseitige "Wilcoxon-Rangsummentest" für Summen, Differenzen und crossoverDifferenzen angewandt („two-period crossover analysis Wilcoxon-Test“). Dies ist ein parameterfreier, statistischer Test für unverbundene Stichproben. Das Testziel ist die Prüfung der Abweichung des Mittelwertes von einem Standardwert. Der Wilcoxon-Test bezieht sich nicht auf die Stichprobenelemente selbst, sondern nur auf deren Rangordnung. Überprüft wird, ob eine Signifikanz in der Übereinstimmung zweier Verteilungen besteht (Koch 1972). Das Signifikanzniveau wurde in der Studie als $\alpha=0,05$ (zweiseitig) definiert. Zum Test auf Unterschiedlichkeit der Behandlungseffekte wurde die Nullhypothese $\left(\mathrm{H}_{0}\right.$ : Effekt unter Behandlung mit Etoricoxib $=$ Effekt unter Behandlung mit Placebo) gegen die Alternativhypothese $\left(\mathrm{H}_{\mathrm{A}}\right.$ : Effekt unter Behandlung mit Etoricoxib $\neq$ Effekt unter Behandlung mit Placebo) getestet.

Das mögliche Auftreten einer Übertragung des Behandlungseffekts („carry-over effect") von der ersten in die zweite Studienphase wurde in einem Vorschalttest überprüft. Hierzu wurde die Summe der Messwerte aus beiden Phasen betrachtet und ein unverbundener Vergleich (Wilcoxon-Rangsummentest) der Sequenzgruppen durchgeführt (Wellek und Blettner 2012). Die Auswertung bezog sich in der Studie auf die Daten der ITT-Population. Fehlten Daten aus den Nachbeobachtungsvisiten, so wurden sie nach der „last observation carried forward"-Methode (LOCF) ersetzt. Das heißt, dass der letzte von einem Probanden vorliegende Wert für die Endauswertung genutzt wurde.

Es ist keine gesonderte Methode bekannt, die eine Berechnung der benötigten Stichprobengröße für den Wilcoxon-Rangsummentest ermöglicht. Aus diesem Grund wurde das Verfahren, welches beim „t-Test“ zur Fallzahlberechnung für unverbundene Stichproben herangezogen wird, verwendet (Röhrig et al. 2010). 
Dabei wurde eine standardisierte Differenz von 0,5 (mittelgroße Differenz nach Cohen) und eine standardisierte Korrelation von 0,5 (mittelgroße Korrelation nach Rho) zwischen den beiden Phasen der crossover-Studie angenommen. $\alpha$ und $\beta$ wurden wie folgt festgelegt: $\alpha=0,025$ (einseitig) und $\beta=0,1$. Das entsprechende Ergebnis der Stichprobengrößenberechnung war $N=44$. Unter Berücksichtigung von möglichen „drop-outs“ und anderer Eventualitäten wurde die Stichprobengröße auf N = 50 erhöht.

Um eine Vergleichbarkeit der Daten zu gewährleisten, wurde die Homogenität der Ausgangswerte der demographischen Daten und der Wirksamkeitskriterien zu Beginn der Studie analysiert. Zu dieser Auswertung wurde zuerst der multivariate „Wilcoxon-Mann-Whitney Test“ angewandt. Dies ist ein nichtparametrischer, statistischer Rangordnungs-Test zweier unabhängiger Stichproben. Mit diesem Test wird geprüft, ob zwei Merkmale die gleiche Größenordnung besitzen. Der Test wurde in Kombination mit dem „Wei-Lachin Verfahren“ angewandt (Lachin 1992).

In der Auswertung kamen verschiedene PC-Software zum Einsatz. Die Berechnung der Stichprobengröße erfolgte mit der Software „N“ (Version 2.2, idv/Gauting). Zur Datenanalyse wurden die Software „Testimate“ (Version 6.5.0, idv/Gauting) und „Excel“ (Version 12.0, Microsoft) verwendet. 


\section{ERGEBNISSE}

\subsection{Demographische Daten}

Die demographischen Daten (Geschlecht, Alter, Body-Mass-Index (BMI), Temperatur, Blutdruck und Herzfrequenz) der jeweiligen Behandlungsgruppe zu Beginn von Phase 1 sind in Tab. 4 zusammengefasst.

Tab. 4: Demographische Daten der ITT/PP-Population zu Beginn von Phase 1

\begin{tabular}{|c|c|c|c|c|}
\hline \multicolumn{3}{|c|}{ Behandlungsgruppe in Phase 1} & Etoricoxib & Placebo \\
\hline \multirow{4}{*}{$\begin{array}{l}\text { Anzahl der } \\
\text { Probanden }\end{array}$} & Gesamt & Anzahl & 24 & 24 \\
\hline & Männlich & $\begin{array}{l}\text { Anzahl } \\
(\%)\end{array}$ & $14(58,3)$ & $11(45,8)$ \\
\hline & Weiblich & $\begin{array}{l}\text { Anzahl } \\
(\%)\end{array}$ & $10(41,7)$ & $13(54,2)$ \\
\hline & \multicolumn{2}{|l|}{ P-Wert } & \multicolumn{2}{|l|}{0,5639} \\
\hline \multirow[t]{3}{*}{ Alter [Jahre] } & \multicolumn{2}{|c|}{ Mittelwert (SA) } & $44,2(16,3)$ & $46,6(16,3)$ \\
\hline & \multicolumn{2}{|c|}{ Min-Median-Max } & $22-45-69$ & $23-52,5-70$ \\
\hline & \multicolumn{2}{|l|}{ P-Wert } & \multicolumn{2}{|l|}{0,5158} \\
\hline \multirow[t]{3}{*}{ BMI } & \multicolumn{2}{|c|}{ Mittelwert (SA) } & $25,5(2,67)$ & $24,7(3,09)$ \\
\hline & \multicolumn{2}{|c|}{ Min-Median-Max } & $21-25-30$ & 20-24-31 \\
\hline & \multicolumn{2}{|l|}{ P-Wert } & \multicolumn{2}{|l|}{0,2693} \\
\hline \multirow{2}{*}{$\begin{array}{l}\text { Temperatur } \\
{\left[{ }^{\circ} \mathrm{C}\right]}\end{array}$} & \multicolumn{2}{|c|}{ Mittelwert (SA) } & $36,23(0,61)$ & $36,68(0,27)$ \\
\hline & \multicolumn{2}{|c|}{ Min-Median-Max } & $35.0-36,35-36.9$ & $36,0-36,7-37.0$ \\
\hline \multirow{2}{*}{$\begin{array}{l}\text { Systolicher } \\
\text { RR [mmHg] }\end{array}$} & \multicolumn{2}{|c|}{ Mittelwert (SA) } & $128,7(8,6)$ & $132,0(10,3)$ \\
\hline & \multicolumn{2}{|c|}{ Min-Median-Max } & $118-128-155$ & $107-133,5-149$ \\
\hline \multirow{2}{*}{$\begin{array}{l}\text { Diastolicher } \\
\mathrm{RR} \text { [mmHg] }\end{array}$} & \multicolumn{2}{|c|}{ Mittelwert (SA) } & $81,3(8,5)$ & $82,3(9,0)$ \\
\hline & \multicolumn{2}{|c|}{ Min-Median-Max } & $65-80,5-98$ & $58-82-102$ \\
\hline
\end{tabular}




\begin{tabular}{|l|l|l|l|}
\hline \multicolumn{2}{|l|}{ Behandlungsgruppe in Phase 1 } & Etoricoxib & Placebo \\
\hline $\begin{array}{l}\text { Herzfrequenz } \\
\text { [Schläge/min] }\end{array}$ & Mittelwert (SA) & $71,5(14,1)$ & $64,5(12,3)$ \\
\hline & Min-Median-Max & $43-73-95$ & $49-62-97$ \\
\hline
\end{tabular}

Die demographischen Daten der beiden Behandlungsgruppen zu Beginn der Studie sind vergleichbar. Die Etoricoxib-Gruppe zeigte im Vergleich einen statstisch nicht signifikant erhöhten BMI ( $p=0,2693)$ (siehe Abb. 2).

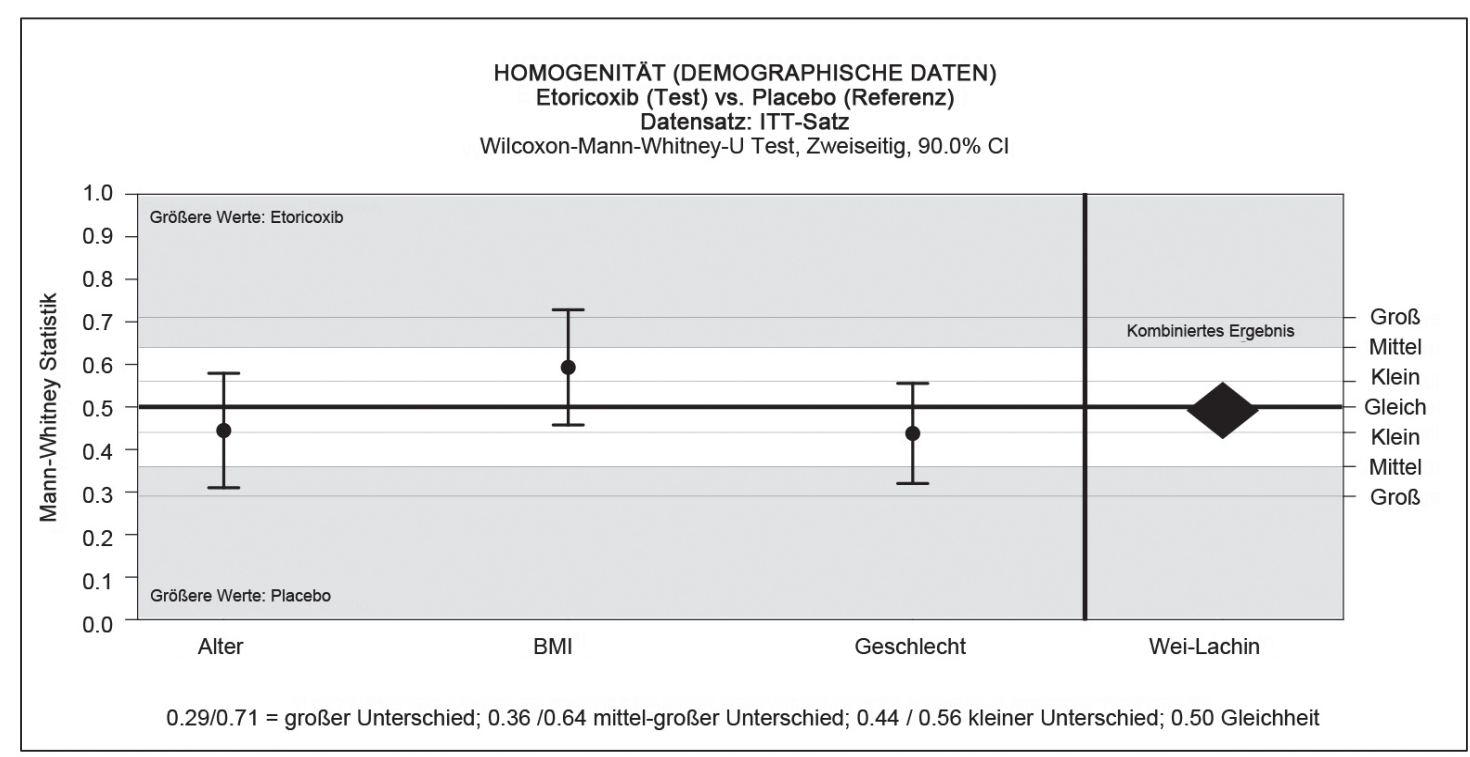

Abb. 2: Wilcoxon-Mann-Whitney Statistik - Homogenitätsanalyse der demographischen Daten zu Beginn der Studie

\section{2 $\mathrm{H}_{1}$ : Etoricoxib bei Muskelschmerz nach exzentrischer Belastung}

Im Folgenden werden die Ergebnisse zur Prüfung der Arbeitshypothese $\mathrm{H}_{1}$ dargestellt: Es gibt keinen Unterschied zwischen der Wirksamkeit von Etoricoxib und Placebo auf Muskelschmerz, auf die Erholung der Muskelkraft und auf Entzündungsmarker im Blut nach exzentrischer Belastung.

Die durch das exzentrische Belastungsmodell erzeugten Veränderungen der Wirksamkeitskriterien (Ruheschmerz, Bewegungsschmerz, Schmerzschwelle am max. Druckpunkt, Maximalkraft und Entzündungsmarker) wurden mit den 
entsprechenden Ausgangswerten vor exzentrischer Belastung verglichen, um den zeitlichen Verlauf von DOMS zu beschreiben. Zur Bewertung von Ruheschmerz und Maximalkraft vor und zu Zeitpunkten nach exzentrischer Belastung wurde der "t-Test" zum Vergleich von Mittelwertunterschieden angewandt. Als Ausgangswert für Ruheschmerz wurde ein Wert von NRS 0 angenommen. Als Ausgangswert für Maximalkraft wurden die erhobenen Werte während des Screening herangezogen. Die zeitlichen Verläufe von Bewegungsschmerz, Schmerzschwelle am max. Druckpunkt und Entzündungsparameter wurden aufgrund fehlender Ausgangswerte nur beschreibend ausgewertet.

Zur Prüfung der Arbeitshypothese werden die Veränderungen der Wirksamkeitskriterien hinsichtlich der Behandlung mit Etoricoxib und Placebo gegenübergestellt. Zur Bewertung aller Wirksamkeitskriterien wurde der „two-period crossover analysis Wilcoxon-Test" (zweiseitiger Test für die Differenz) für Summen, Differenzen und crossover Differenzen angewandt. Verglichen wurde die Etoricoxib-Gruppe mit der Placebo-Gruppe.

\subsubsection{Schmerz}

Die Ausgangswerte für Ruheschmerz ( $p=0,7443)$ und Bewegungsschmerz ( $p$ $=0,7443)$ zeigten in der Homogenitätsanalyse keine statistisch signifikanten Unterschiede und waren somit zwischen beiden Behandlungsgruppen vergleichbar.

Abb. 3 und Abb. 4 stellen die Summen aller auf der NRS abgegebenen Bewertungen von Ruheschmerz bzw. Bewegungsschmerz nach exzentrischer Belastung in den Behandlungsgruppen gegenüber. Die Etoricoxib-Gruppe (Mittelwert: 10,4 $\pm 9,7$ ) zeigte im Vergleich zur Placebo-Gruppe (Mittelwert: 11,5 $\pm 9,9$ ) in der Summe einen statistisch nicht signifikant niedrigeren Schmerz in Ruhe ( $p$ $=0,5837)$. Der Bewegungsschmerz war in der Summe in der Etoricoxib-Gruppe (Mittelwert: 44,5 \pm 14,4) im Vergleich zur Placebo-Gruppe (Mittelwert: 44,0 \pm $14,0)$ nicht signifikant erhöht $(p=0,8634)$. 


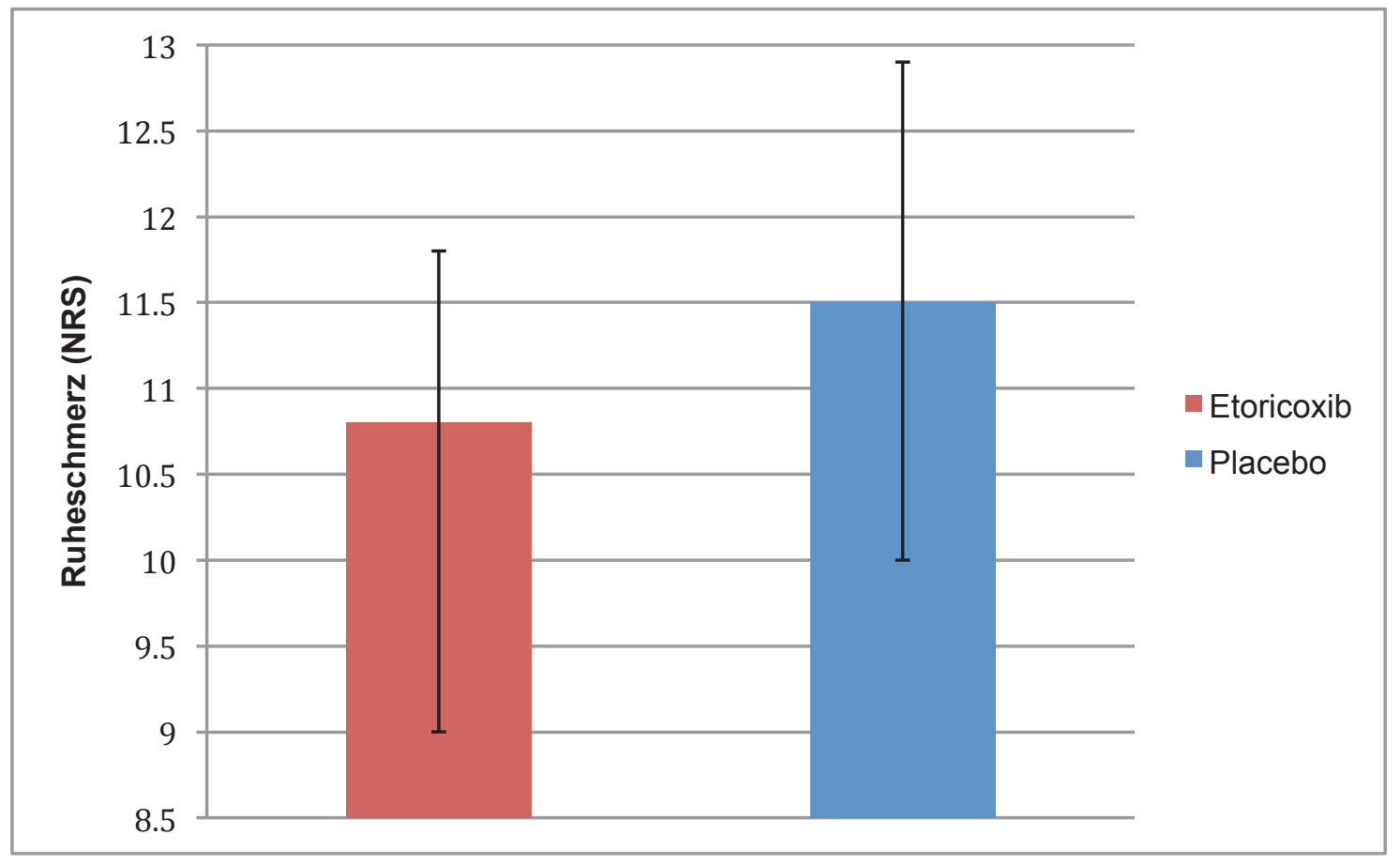

Abb. 3: Summe aller Bewertungen - Ruheschmerz nach exzentrischer Belastung über den gesamten Nachbeobachtungszeitraum von 8 Tagen

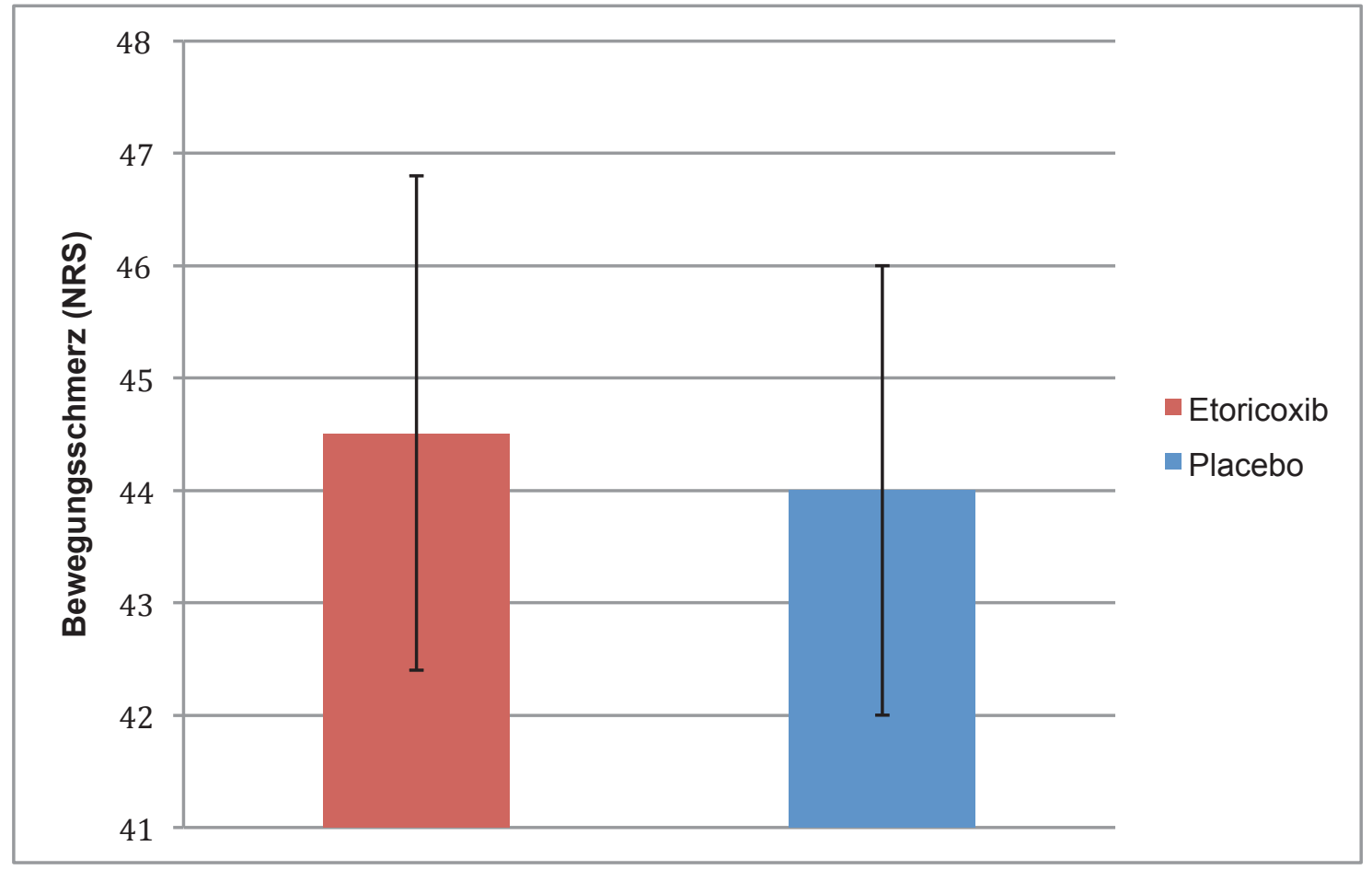

Abb. 4: Summe aller Bewertungen - Bewegungsschmerz nach exzentrischer Belastung über den gesamten Nachbeobachtungszeitraum von 8 Tagen 


\subsubsection{Ruheschmerz}

Abb. 5 stellt den zeitlichen Verlauf des Ruheschmerzes nach exzentrischer Belastung für die Etoricoxib- und die Placebo-Gruppe dar.

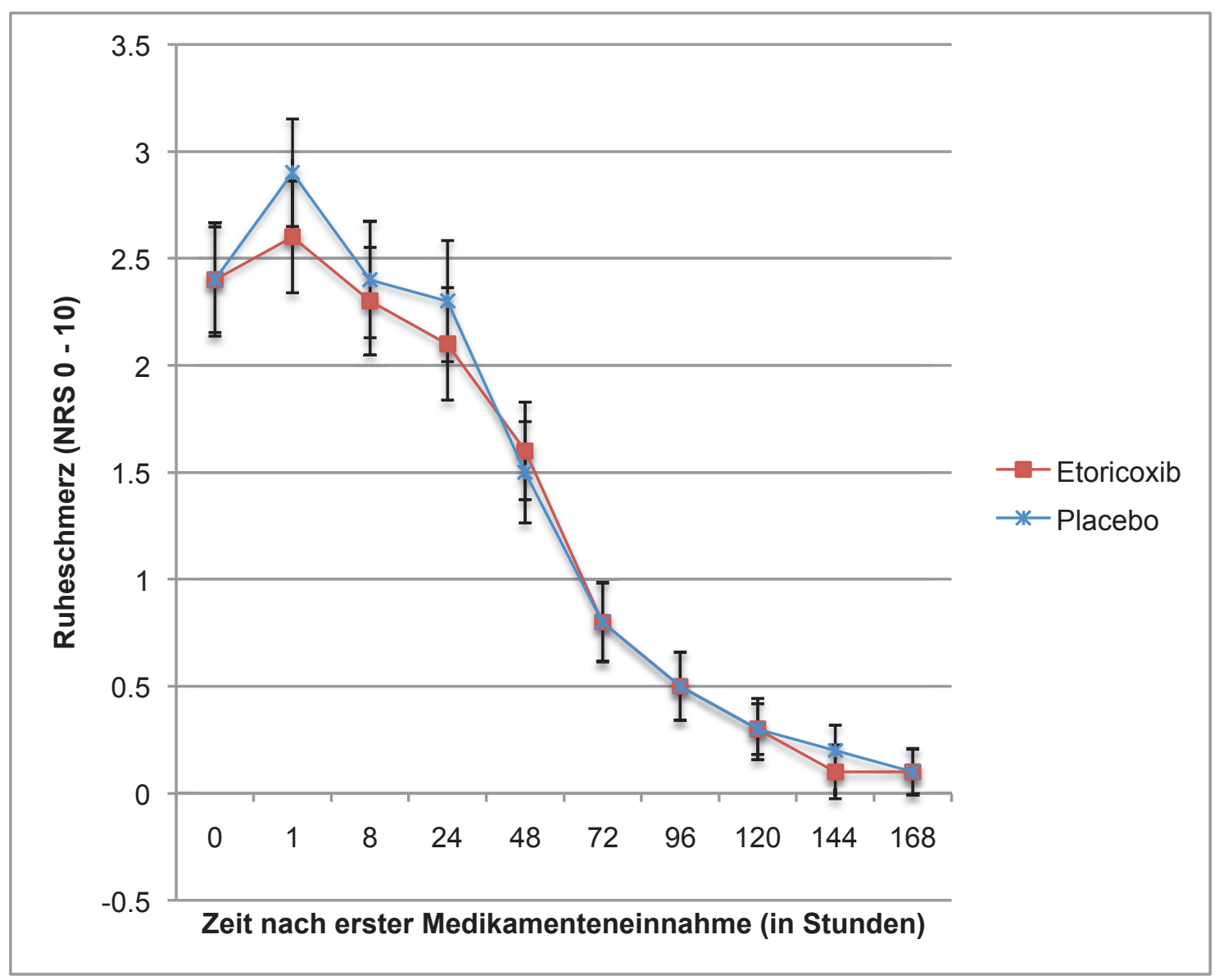

Abb. 5: Zeitlicher Verlauf - Ruheschmerz nach exzentrischer Belastung unter Behandlung mit Etoricoxib vs. Placebo (Mittelwert \pm SEM)

$16 \pm 2$ Stunden nach der exzentrischen Belastung zeigte sich in beiden Behandlungsgruppen ein statistisch signifikant erhöhter Ruheschmerz $(p<0,05)$. Innerhalb der ersten 40 Stunden nach exzentrischer Belastung (entsprechend innerhalb der ersten 24 Stunden nach Behandlungsbeginn) wurden maximale Werte für Schmerz in Ruhe (NRS 2-3) erreicht. Der Ruheschmerz nahm eine Stunde nach erster Medikamenteneinnahme beginnend in beiden Behandlungsgruppen kontinuierlich ab. Bis 120 Stunden nach nach Behandlungsbeginn blieb in beiden Behandlungsgruppen ein statistisch signifikant erhöhter Ruhe- 
schmerz im Vergleich zum Ausgangswert bestehen (Etoricoxib-Gruppe: $p=$ 0,026; Placebo-Gruppe: $p=0,001)$. Ab 140 Stunden nach nach Behandlungsbeginn konnte in beiden Behandlungsgruppen kein statistisch signifikant erhöhter Ruheschmerz mehr demonstriert werden (Etoricoxib-Gruppe: $p=0,254$; Placebo-Gruppe: $p=0,077$ ) und die Werte erreichten wieder das Ausgangsniveau (NRS 0-0,2).

Abb. 6 stellt den zeitlichen Verlauf des Ruheschmerzes innerhalb der ersten 24 Stunden nach Behandlungsbeginn für die Etoricoxib- und die Placebo-Gruppe dar.

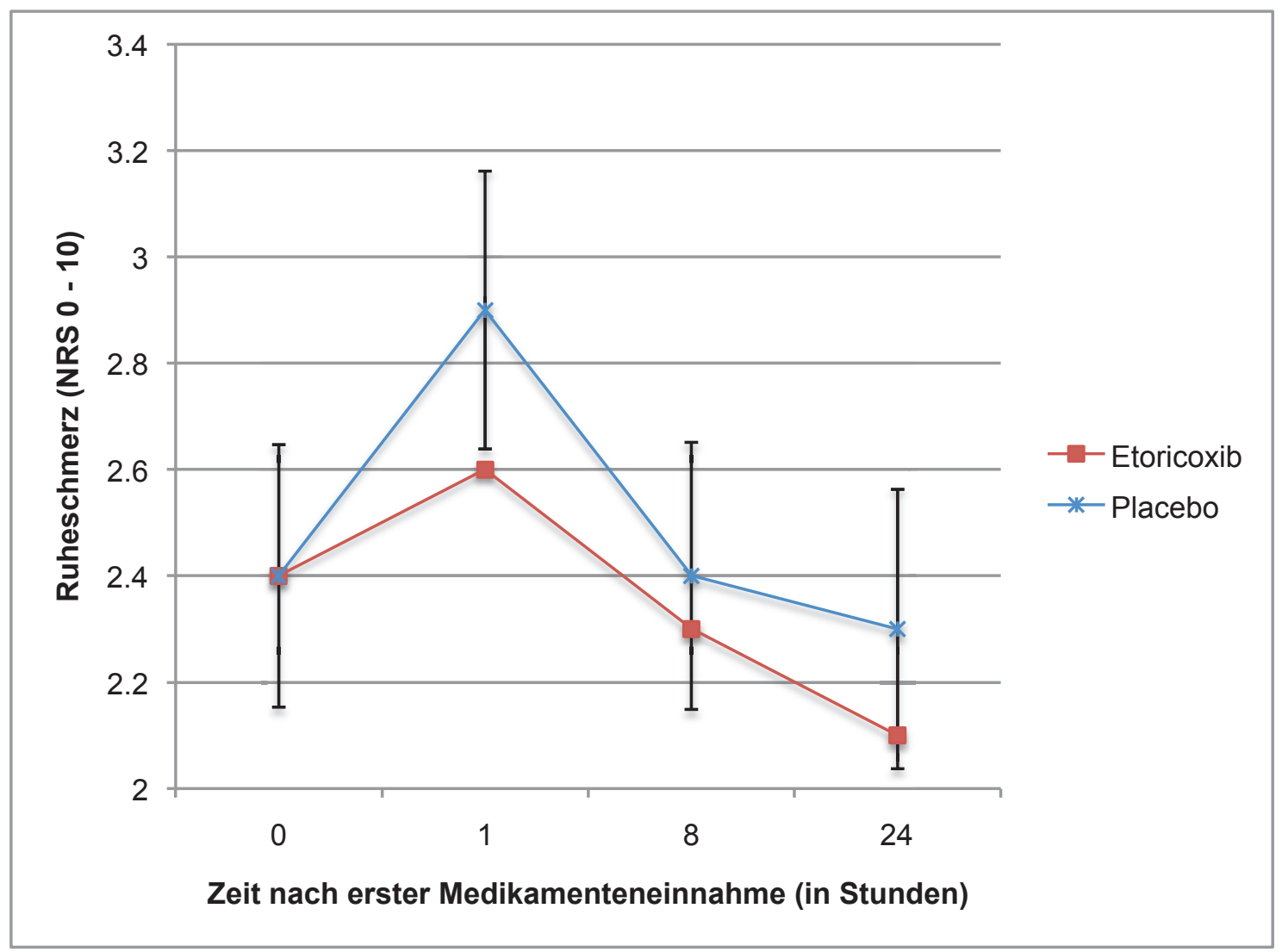

Abb. 6: Zeitlicher Verlauf - Ruheschmerz unter Behandlung mit Etoricoxib vs. Placebo (Mittelwert \pm SEM) innerhalb der ersten 24 Stunden nach Behandlungsbeginn

Tab. 5: Beschreibende Statistik - Ruheschmerz (Phase 1 und 2)

\begin{tabular}{|l|l|l|}
\hline & Etoricoxib & Placebo \\
\hline $\mathrm{N}$ & 48 & 48 \\
\hline
\end{tabular}




\begin{tabular}{|l|l|l|l|}
\hline \multicolumn{2}{|l|}{} & Etoricoxib & Placebo \\
\hline $\begin{array}{l}\text { Summe aller Bewer- } \\
\text { tungen }\end{array}$ & Mittelwert (SA) & $10,4(9,7)$ & $11,5(9,9)$ \\
\hline & Min-Median-Max & $0,0-8,5-52,0$ & $0,0-9,0-53,0$ \\
\hline Erste 24 Stunden & Mittelwert (SA) & $7,0(5,0)$ & $7,6(5,2)$ \\
\hline & Min-Median-Max & $0,0-6,5-20,0$ & $0,0-7,0-21,0$ \\
\hline Tag 3-Tag 8 & Mittelwert (SA) & $3,4(5,4)$ & $3,6(5,7)$ \\
\hline & Min-Median-Max & $0,0-1,0-32,0$ & $0,0-2,0-36,0$ \\
\hline
\end{tabular}

In der Auswertung der crossover-Analyse zeigte sich ein statistisch nicht signifikanter Trend zu einer Reduktion von Ruheschmerz nach exzentrischer Belastung unter der Behandlung mit Etoricoxib $(p=0,1341)$, insbesondere innerhalb der ersten 24 Stunden nach Behandlungsbeginn. Kein statistisch signifikanter Übertragungseffekt wurde demonstriert $(p=0,5845)$ (siehe Tab. 6).

Tab. 6: Two-period crossover-Analyse - Vergleich der Behandlungsgruppen Etoricoxib vs. Placebo hinsichtlich Ruheschmerz

Test Resultate Übertragung Behandlung Phase

Wilcoxon T

(Sequenz A-B / B-A) $\quad 561,5 / 614,5 \quad 660,5 / 515,5 \quad 573,5 / 602,5$

Asymptotisch unkorrigiert $\quad(\mathrm{DF}=1)$

$\begin{array}{llll}X^{2} & 0,2990 & 2,2444 & 0,0897 \\ \text { P-Wert } & 0,5845 & 0,1341 & 0,7645 \\ \text { er P-Wert } & 0,5913 & 0,1364 & 0,7705\end{array}$

Geschätzter Korrelationskoeffizient (Rho): 0,73

Test für Behandlungseffekt nur in Phase 1:

Asymp. P-Wert $=0,3969\left(X^{2}=0,7176, \mathrm{DF}=1\right)$

Exakter P-Wert $=0,4033$ 


\subsubsection{Bewegungsschmerz}

Abb. 7 stellt den zeitlichen Verlauf von Bewegungsschmerz für die Etoricoxibund die Placebo-Gruppe dar.

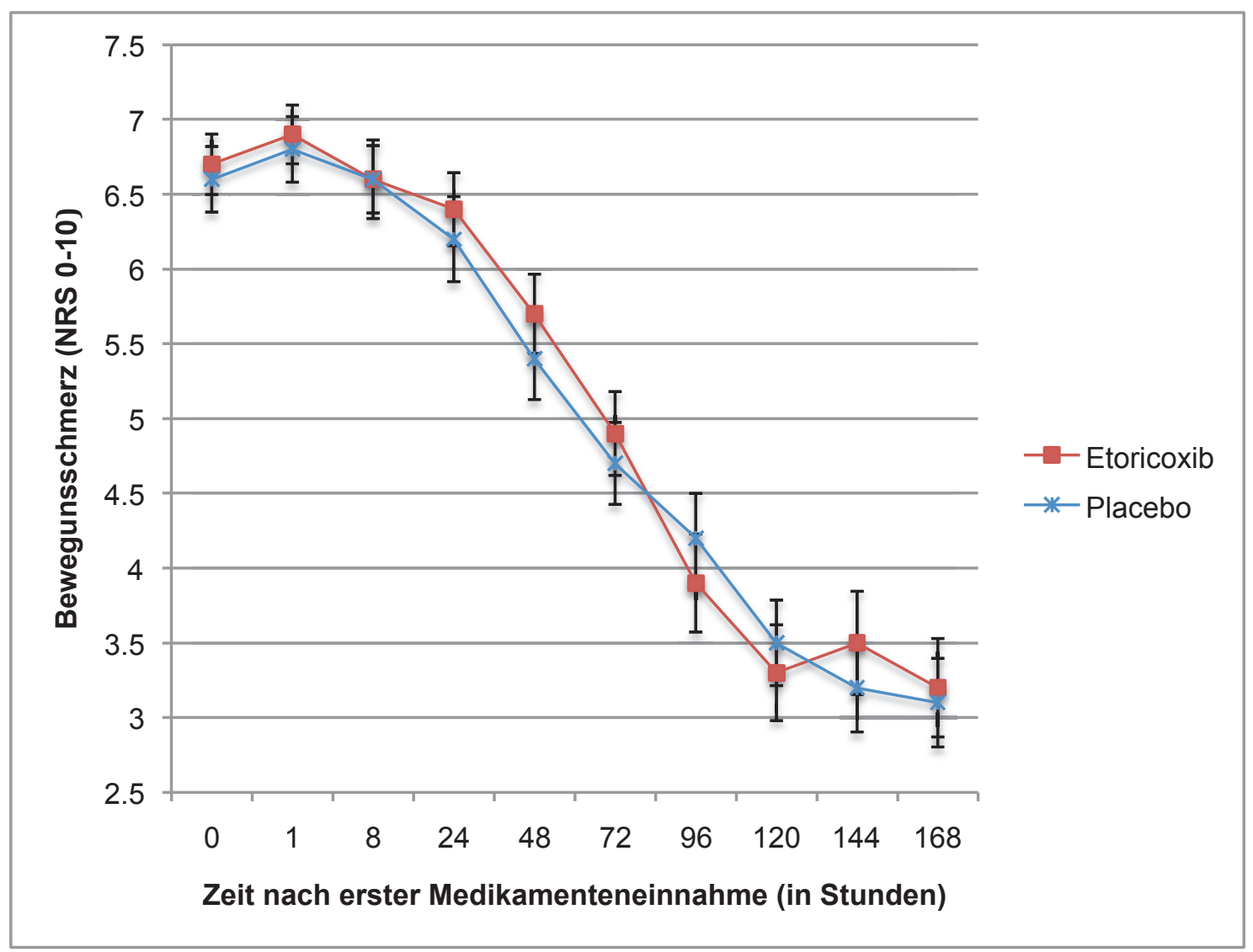

Abb. 7: Zeitlicher Verlauf - Bewegungsschmerz unter Behandlung mit Etoricoxib vs. Placebo (Mittelwert \pm SEM)

$16 \pm 2$ Stunden nach der exzentrischen Belastung zeigte sich in beiden Behandlungsgruppen ein erhöhter Bewegungsschmerz. Maximale Werte für Bewegungsschmerz (NRS 6,5-7) traten innerhalb der ersten 40 Stunden nach exzentrischer Belastung (entsprechend innerhalb der ersten 24 Stunden nach Behandlungsbeginn) auf. Der Bewegungsschmerz nahm eine Stunde nach erster Medikamenteneinnahme beginnend in beiden Behandlungsgruppen kontinuierlich ab. Bis 96 Stunden nach Behandlungsbeginn blieb in beiden Behandlungsgruppen ein erhöhter Ruheschmerz bestehen. Ab 120 Stunden nach nach 
Behandlungsbeginn pendelten sich die Werte für Bewegungsschmerz in beiden Behandlungsgruppen auf einem leicht erhöhten Niveau (NRS 3-3.5) ein.

Tab. 7: Beschreibende Statistik - Bewegungsschmerz (Phase 1 und 2)

\begin{tabular}{|c|c|c|c|}
\hline & & Etoricoxib & Placebo \\
\hline \multicolumn{2}{|l|}{$\mathrm{N}$} & 48 & 48 \\
\hline \multirow{2}{*}{$\begin{array}{l}\text { Summe aller Bewer- } \\
\text { tungen }\end{array}$} & Mittelwert (SA) & $44,5(14,4)$ & $44,0(14,0)$ \\
\hline & Min-Median-Max & $8,0-42,5-71,0$ & $18,0-41,5-74,0$ \\
\hline \multirow[t]{2}{*}{ Erste 24 Stunden } & Mittelwert (SA) & $19,9(4,1)$ & $19,6(4,9)$ \\
\hline & Min-Median-Max & $7,0-20,0-27,0$ & $10,0-19,0-27,0$ \\
\hline \multirow[t]{2}{*}{ Tag 3-Tag 8} & Mittelwert (SA) & $24,6(11,7)$ & $24,1(10,5)$ \\
\hline & Min-Median-Max & $1,0-23,0-48,0$ & $7,0-21,0-49,0$ \\
\hline
\end{tabular}

In der Auswertung der crossover-Analyse zeigten sich keine statistisch signifikanten Behandlungseffekte unter Etoricoxib ( $p=0,7257)$. Weder die gesamte Studienphase ( $p=0,7257)$, noch Phase 1 für sich betrachtet $(p=0,4576)$ zeigten eine Wirksamkeit der Therapie. Kein signifikanter Übertragungseffekt konnte demonstriert werden $(p=0,2789)$ (siehe Tab. 8).

Tab. 8: Two-period crossover-Analyse - Vergleich der Behandlungsgruppen Etoricoxib vs. Placebo hinsichtlich Bewegungsschmerz

Test Resultate

Übertragung

Behandlung

Phase

Wilcoxon T

(Sequenz A-B / B-A) $\quad 535,5 / 640,5 \quad 571,0$ / 605,0 $\quad 591,5 / 584,5$

Asymptotisch unkorrigiert $\quad(\mathrm{DF}=1)$

$\begin{array}{llll}X^{2} & 1,1726 & 0,1231 & 0,0052 \\ \text { P-Wert } & 0,2789 & 0,7257 & 0,9424\end{array}$

Exakter P-Wert

$\begin{array}{lll}0,2840 & 0,7319 & 0,9470\end{array}$

Geschätzter Korrelationskoeffizient (Rho): 0,57

Test für Behandlungseffekt nur in Phase 1: 
Asymp. P-Wert $=0,4576\left(X^{2}=0,5516\right.$, DF $\left.=1\right)$

Exakter P-Wert $=0,4643$

\subsubsection{Schmerzschwelle am max. Druckpunkt}

Abb. 8 stellt die Veränderung der Hitzeschmerzschwelle am max. Druckpunkt nach exzentrischer Belastung für die Etoricoxib-Gruppe und die PlaceboGruppe im Verlauf dar.

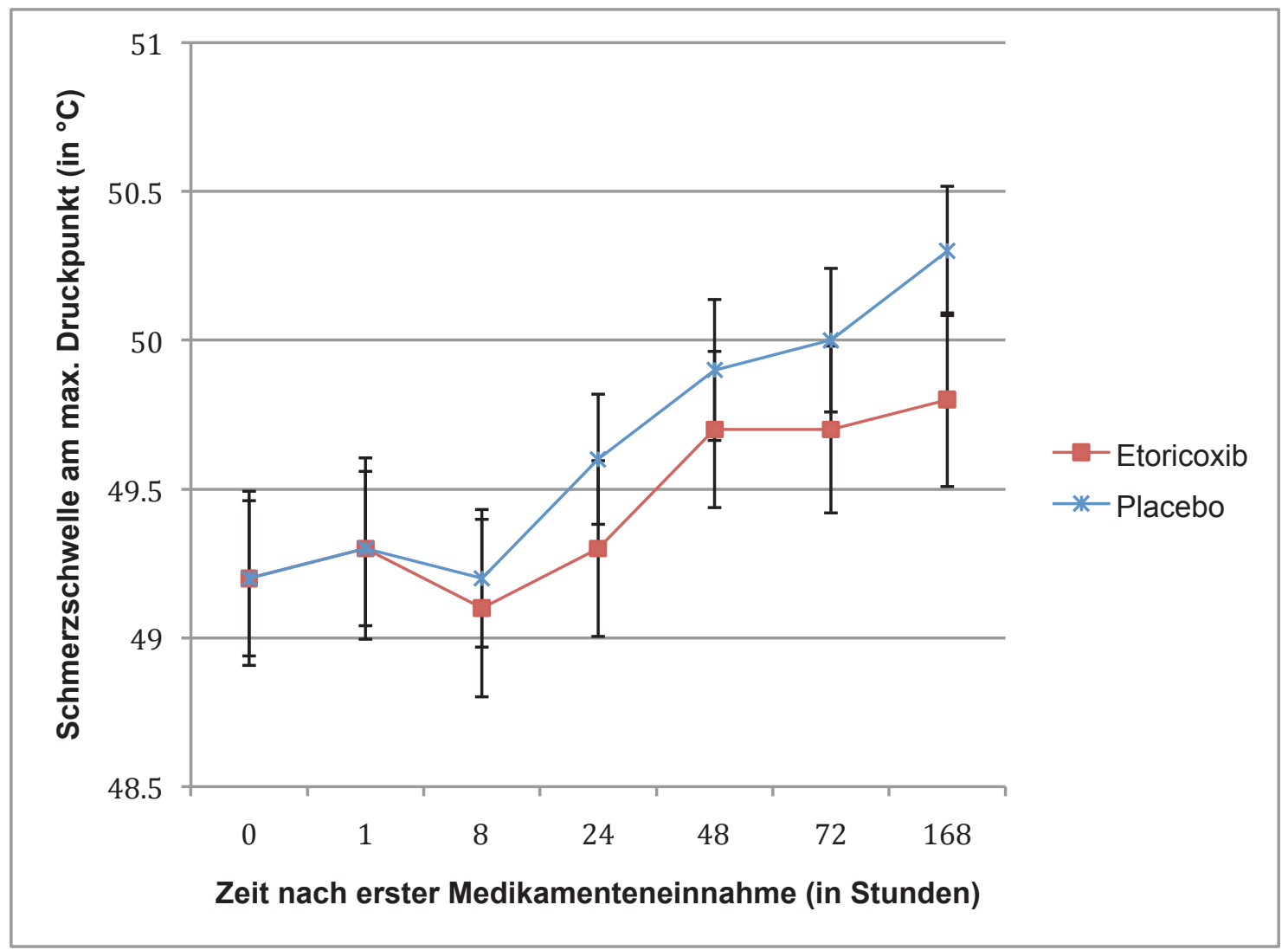

Abb. 8: Zeitlicher Verlauf - Hitzeschmerzschwelle am max. Druckpunkt unter Behandlung mit Etoricoxib vs. Placebo (Mittelwert \pm SEM)

Die Schmerzschwelle zeigte sich in beiden Behandlungsgruppen $16 \pm 2$ Stunden nach der exzentrischen Belastung gleichermaßen erniedrigt. Ab Stunde 8 nach Behandlungsbeginn stieg die Schmerzschwelle beider Behandlungsgruppen wieder kontinuierlich an. Beginnend 8 Stunden nach Behandlungsbeginn zeigte sich eine leicht bis moderat erniedrigte Schmerzschwelle der Etoricoxib- 
Gruppe im Vergleich zur Placebo-Gruppe, die bis zum Ende der Nachbeobachtungsphase 168 Stunden nach Behandlungsbeginn bestehen blieb (Mittelwert: Etoricoxib-Gruppe 49,8; Placebo-Gruppe 50,3). Es zeigte sich der Trend zu einer verzögerten Wiederherstellung der ursprünglichen Schmerzschwelle unter Etoricoxib-Behandlung.

Tab. 9: Beschreibende Statistik - Hitzeschmerzschwelle am Druckpunkt (Phase 1 und 2)

\begin{tabular}{|c|c|c|c|}
\hline & & Etoricoxib & Placebo \\
\hline \multicolumn{2}{|l|}{$\mathrm{N}$} & 44 & 43 \\
\hline \multirow{2}{*}{$\begin{array}{l}\text { Summe aller Be- } \\
\text { wertungen }\end{array}$} & Mittelwert (SA) & $296,4(10,8)$ & $298,4(9,0)$ \\
\hline & Min-Median-Max & $\begin{array}{l}274,0-198,6- \\
312,0\end{array}$ & $\begin{array}{l}271,5-300,0- \\
312,0\end{array}$ \\
\hline \multirow[t]{2}{*}{ Erste 24 Stunden } & Mittelwert (SA) & $147,7(5,7)$ & $148,1(4,6)$ \\
\hline & Min-Median-Max & $\begin{array}{l}133,9-138,7- \\
156,0\end{array}$ & $\begin{array}{l}135,3-148,0- \\
156,0\end{array}$ \\
\hline \multirow[t]{2}{*}{ Tag 3-Tag 8} & Mittelwert (SA) & $149,0(5,1)$ & $150,3(4,5)$ \\
\hline & Min-Median-Max & $\begin{array}{l}138,6-149,6- \\
156,0\end{array}$ & $\begin{array}{l}136,2-152,0- \\
156,0\end{array}$ \\
\hline
\end{tabular}

Die Auswertung der crossover-Analyse erbrachte keinen Nachweis für einen statistisch signifikanten Unterschied zwischen den beiden Behandlungsgruppen $(p=0,4586)$ (siehe Tab. 10).

Tab. 10: Two-period crossover-Analyse - Vergleich der Behandlungsgruppen Etoricoxib vs. Placebo hinsichtlich der Hitzeschmerzschwelle am max. Druckpunkt

Test Resultate Übertragung

Behandlung

Phase

Wilcoxon T

(Sequenz A-B / B-A) $\quad 485,0$ / 461, $\quad 514,5$ / 431, $5 \quad 507,5$ / 438,5

Asymptotisch unkorrigiert $\quad(\mathrm{DF}=1)$

$\begin{array}{llll}X^{2} & 0,0006 & 0,5492 & 0,3261 \\ \text { P-Wert } & 0,9806 & 0,4586 & 0,5680\end{array}$


Test Resultate

Exakter P-Wert

0,9904

0,4662

0,5758

Geschätzter Korrelationskoeffizient (Rho): 0,80

Test für Behandlungseffekt nur in Phase 1:

Asymp. P-Wert $=0,5930\left(\mathrm{X}^{2}=0,2857, \mathrm{DF}=1\right)$

Exakter P-Wert $=0,6004$

\subsubsection{Maximalkraft}

In Abb. 9 ist der zeitliche Verlauf der maximalen Muskelkraft nach exzentrischer Belastung dargestellt.

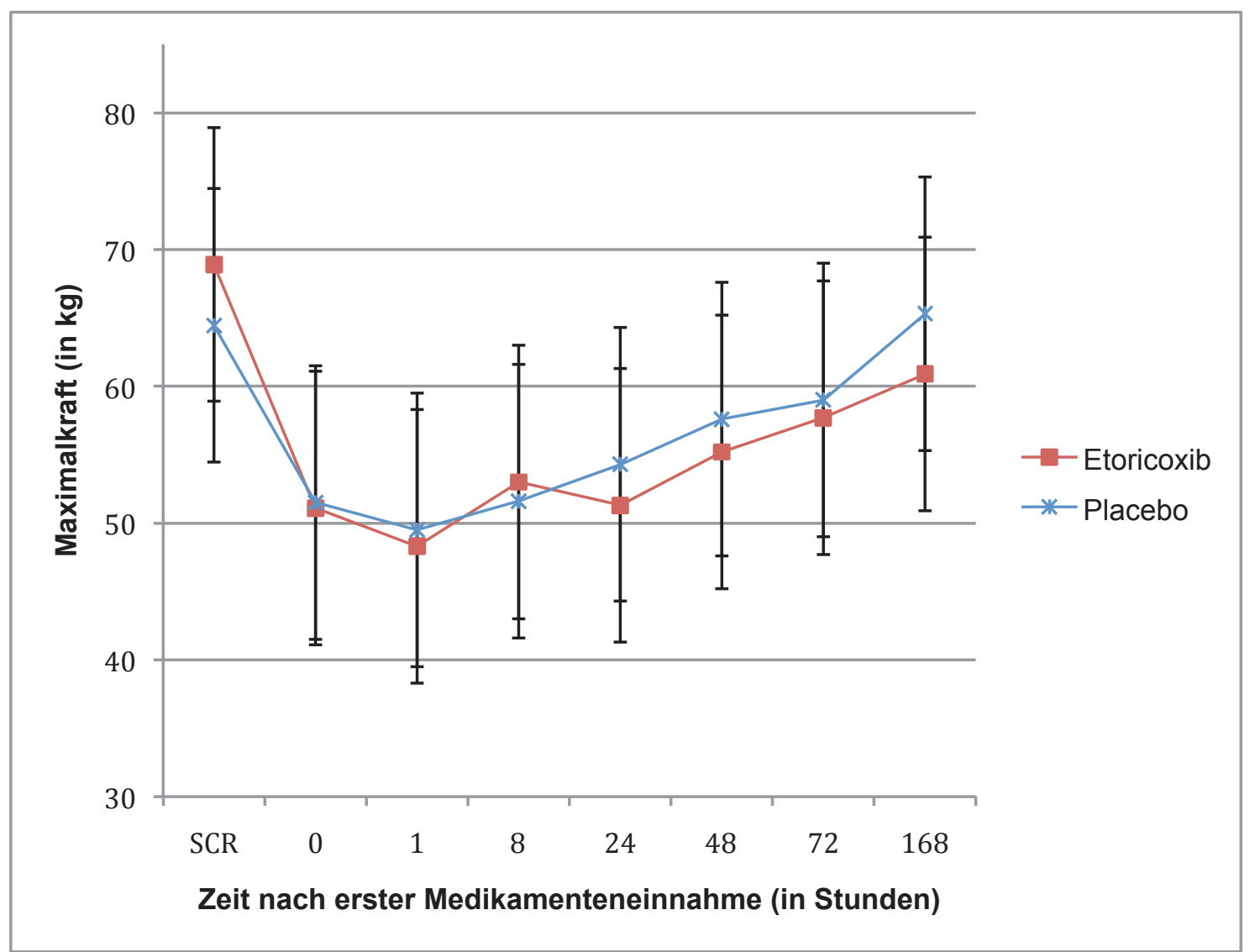

Abb. 9: Zeitlicher Verlauf - Maximalkraft unter Behandlung mit Etoricoxib vs. Placebo (Mittelwert \pm SEM) 
$16 \pm 2$ Stunden nach exzentrischer Belastung zeigte sich in beiden Behandlungsgruppen eine statistisch signifikante Minderung der maximalen Muskelkraft $(p<0,05)$. Eine Stunde nach Behandlungsbeginn (entprechend $17 \pm 2$ Stunden nach exzentrischer Belastung) war die Kraftminderung am ausgeprägtesten. Im Vergleich zu den Ausgangswerten nahm die Muskelkraft durch die exzentrische Belastung in der Etoricoxib-Gruppe um maximal 30,0\% und in der PlaceboGruppe um maximal $23,5 \%$ ab. Danach stieg die maximale Muskelkraft in beiden Behandlungsgruppen wieder kontinuierlich an. In der Placebo-Gruppe blieb die Muskelkraft bis 72 Stunden nach Behandlungsbeginn statistisch signifikant erniedrigt $(p=0,003)$. 168 Stunden nach Behandlungsbeginn war in der Placebo-Gruppe kein statistisch signifikanter Unterschied der Muskelkraft im Vergleich zu den Ausgangswerten mehr zu erkennen $(p=0,879)$. Die maximale Muskelkraft in der Etoricoxib-Gruppe zeigte sich beginnend 24 Stunden nach Behandlungsbeginn im Vergleich zur Placebo-Gruppe leicht erniedrigt. Im Vergleich zu den Ausgangswerten konnte in der Etoricoxib-Gruppe auch 168 Stunden nach Behandlungsbeginn noch eine statistisch signifikant reduzierte Maximalkraft $(p=0,045)$ demonstriert werden. Im Gegensatz zur Placebo-Gruppe konnte die vor exzentrischer Belastung bestehende maximale Muskelkraft unter Etoricoxib-Behandlung auch 8 Tage nach der exzentrischen Belastung nicht wiederhergestellt werden.

Tab. 11: Beschreibende Statistik - Maximalkraft (Phase 1 und 2)

\begin{tabular}{|l|l|l|l|}
\hline \multicolumn{2}{|l|}{} & Etoricoxib & Placebo \\
\hline N aller & Mittelwert (SA) & 48 & 47 \\
\hline $\begin{array}{l}\text { Summe } \\
\text { Bewertungen }\end{array}$ & $326,4(130,6)$ & $337,6(159,2)$ \\
\hline & Min-Median-Max & $134,4-300,3-754,3$ & $\begin{array}{l}125,7-295,3- \\
879,7\end{array}$ \\
\hline Erste 24 Stunden & Mittelwert (SA) & $152,6(59,6)$ & $155,6(69,8)$ \\
\hline & Min-Median-Max & $53,9-140,4-325,0$ & $\begin{array}{l}54,7-146,0- \\
429,1\end{array}$ \\
\hline
\end{tabular}




\begin{tabular}{|l|l|l|l|}
\hline \multicolumn{2}{|c|}{} & Etoricoxib & Placebo \\
\hline Tag 3-Tag 8 & Mittelwert (SA) & $173,8(73,0)$ & $182,0(92,2)$ \\
\hline & Min-Median-Max & $73,9-150,1-429,3$ & $\begin{array}{l}62,4-150,8- \\
450,6\end{array}$ \\
\hline
\end{tabular}

Die Auswertung der crossover-Analyse erbrachte keinen Nachweis für einen statistisch signifikanten Unterschied zwischen den beiden Behandlungsgruppen $(p=0,6575)$ (siehe Tab.12).

Tab. 12: Two-period crossover-Analyse - Vergleich der Behandlungsgruppen Etoricoxib vs. Placebo hinsichtlich der Maximalkraft

\begin{tabular}{lcll} 
Test Resultate & Übertragung & Behandlung & Phase \\
\hline Wilcoxon T & & & \\
$\quad \begin{array}{c}\text { (Sequenz A-B / B-A) } \\
\text { Asymptotisch unkorrigiert }\end{array}$ & $630,0 / 546,0$ & $609,5 / 566,5$ & $688,5 / 487,5$ \\
X $^{2}$ & 0,7500 & 0,1966 & 4,2946 \\
P-Wert & 0,3865 & 0,6575 & 0,0382 \\
Exakter P-Wert & 0,3956 & 0,6641 & 0,0379 \\
\hline
\end{tabular}

Geschätzter Korrelationskoeffizient (Rho): 0,83

Test für Behandlungseffekt nur in Phase 1:

$$
\begin{aligned}
& \text { Asymp. P-Wert }=0,3753\left(\mathrm{X}^{2}=0,7861, \mathrm{DF}=1\right) \\
& \text { Exakter P-Wert }=0,3842
\end{aligned}
$$

\subsubsection{Entzündungsmarker}

Die Ausgangswerte für hs CrP waren in der Placebo-Gruppe im Vergleich zur Etoricoxib-Gruppe in der Homogenitätsanalyse signifikant erniedrigt $(p=$ 0,0028) (siehe Abb. 10). Aus diesem Grund wurden lediglich die Änderungen gegenüber den Ausgangswerten in der statistischen Analyse evaluiert. Keine 
signifikanten Unterschiede zeigten sich zwischen den Behandlungsgruppen hinsichtlich der Ausgangswerte für Leukozyten und Sedimentationsrate.

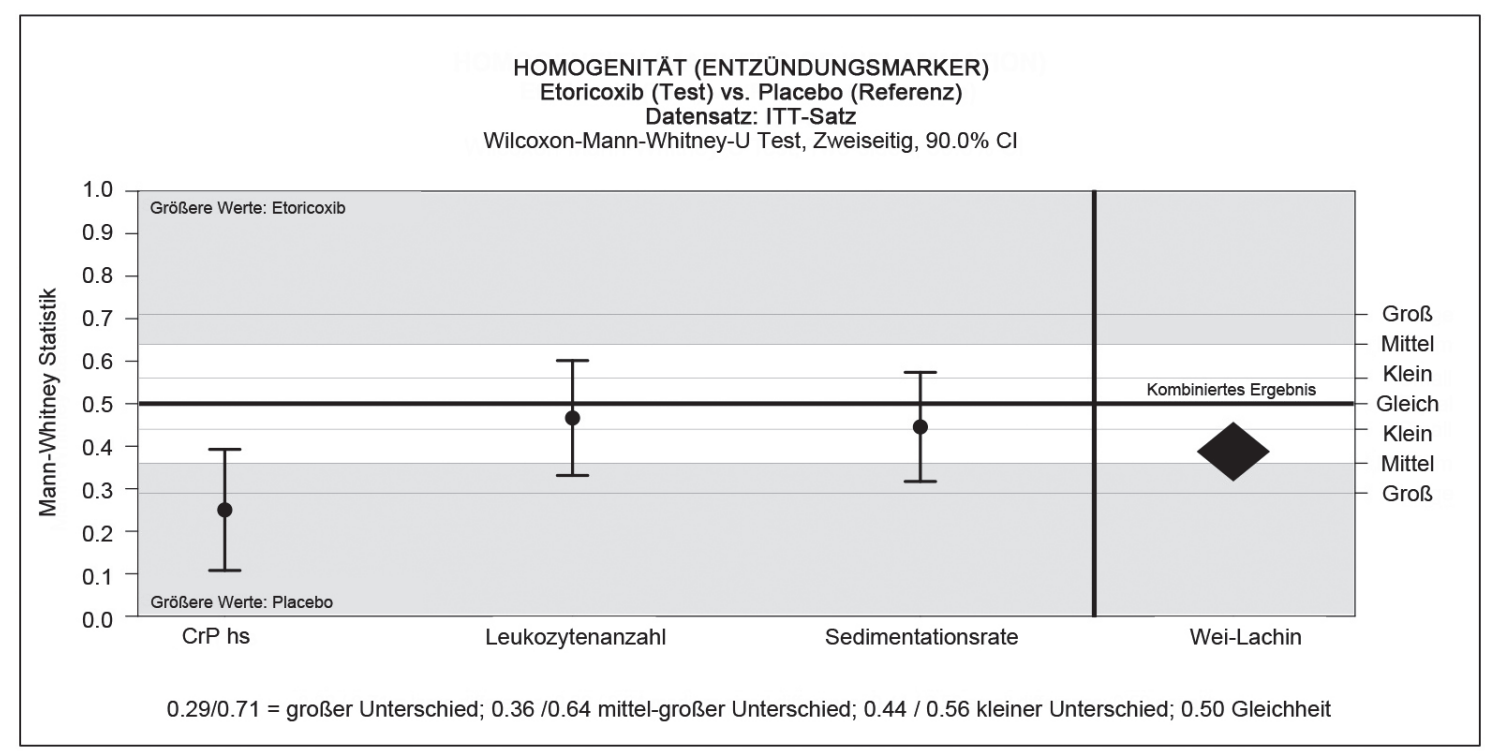

Abb. 10: Wilcoxon-Mann-Whitney Statistik - Homogenitätsanalyse der Ausgangswerte für Entzündungsmarker

\subsubsection{CrP}

Nach der exzentrischen Belastung stiegen die Werte für den Entzündungsmarker hs CrP im Blut an (siehe Tab. 13).

Tab. 13: Beschreibende Statistik - hs CrP-Veränderungen gegenüber den Ausgangswerten (Phase 1 und 2)

\begin{tabular}{|l|l|l|l|}
\hline \multicolumn{2}{|l|}{ Änderung gegenüber Ausgangswerten } & Etoricoxib & Placebo \\
\hline N & 47 & 46 \\
\hline $\begin{array}{l}\text { Summe aller } \\
\text { Bewertungen }\end{array}$ & Mittelwert (SA) & $-0,1493(0,787)$ & $0,084(0,507)$ \\
\hline & Min-Median-Max & $\begin{array}{l}-5,044-(-0,05)- \\
1,371\end{array}$ & $\begin{array}{l}-0,673-0,0015- \\
2,387\end{array}$ \\
\hline Erste 24 Stunden & Mittelwert (SA) & $-0,0087(0,127)$ & $-0,013(0,624)$ \\
\hline & Min-Median-Max & $-0,4-(-0,006)-0,659$ & $-0,336-(-$ \\
& & & $0,0025)-0,104$ \\
\hline Tag 3-Tag 8 & Mittelwert (SA) & $-0,1264(0,691)$ & $0,097(0,536)$ \\
\hline
\end{tabular}




\begin{tabular}{|l|l|l|l|}
\hline \multicolumn{2}{|l|}{ Änderung gegenüber Ausgangswerten } & Etoricoxib & Placebo \\
\hline & Min-Median-Max & $-4,644-(-0,031)-$ & $\begin{array}{l}-0,74-(-0,004)- \\
2,515\end{array}$ \\
& & 0,712 & \\
\hline
\end{tabular}

Die crossover-Analyse demonstrierte einen statistisch signifikanten antientzündlichen Effekt von Etoricoxib in Phase 1 der Studie $(p=0,0341)$. Dieser Effekt wurde in Phase 2 der Studie übertragen. Ein statistisch signifikanter Übertragungseffekt für CrP konnte gezeigt werden ( $p=0,0089$ ). Diese Erkenntnisse stimmen mit dem Gesamtergebnis überein, welches einen statistisch signifikanten antientzündlichen Langzeiteffekt von Etoricoxib im Vergleich zu Placebo anhand der hs CrP-Reduktion bestätigt (siehe Tab. 14 sowie Abb. 11).

Tab. 14: Two-period crossover-Analyse - Vergleich der Behandlungsgruppen Etoricoxib vs. Placebo hinsichtlich hs CrP

\begin{tabular}{cccc} 
Test Resultate & Übertragung & Behandlung & Phase \\
\hline Wilcoxon T & & & \\
\multicolumn{1}{c}{ (Sequenz A-B / B-A) } & $713,5 / 462,5$ & $613,5 / 562,5$ & $590,5 / 585,5$ \\
Asymptotisch unkorrigiert & $(\mathrm{DF}=1)$ & & \\
X $^{2}$ & 6,6969 & 0,2765 & 0,0027 \\
P-Wert & 0,0097 & 0,5990 & 0,9589 \\
Exakter P-Wert & $\mathbf{0 , 0 0 8 9}$ & 0,6058 & 0,9635
\end{tabular}

Geschätzter Korrelationskoeffizient (Rho): 0,14

Test für Behandlungseffekt nur in Segment 1:

Asymp. P-Wert $=0,0345\left(\mathrm{X}^{2}=4,4674, \mathrm{DF}=1\right)$

Exakter P-Wert $\mathbf{= 0 , 0 3 4 1}$ 


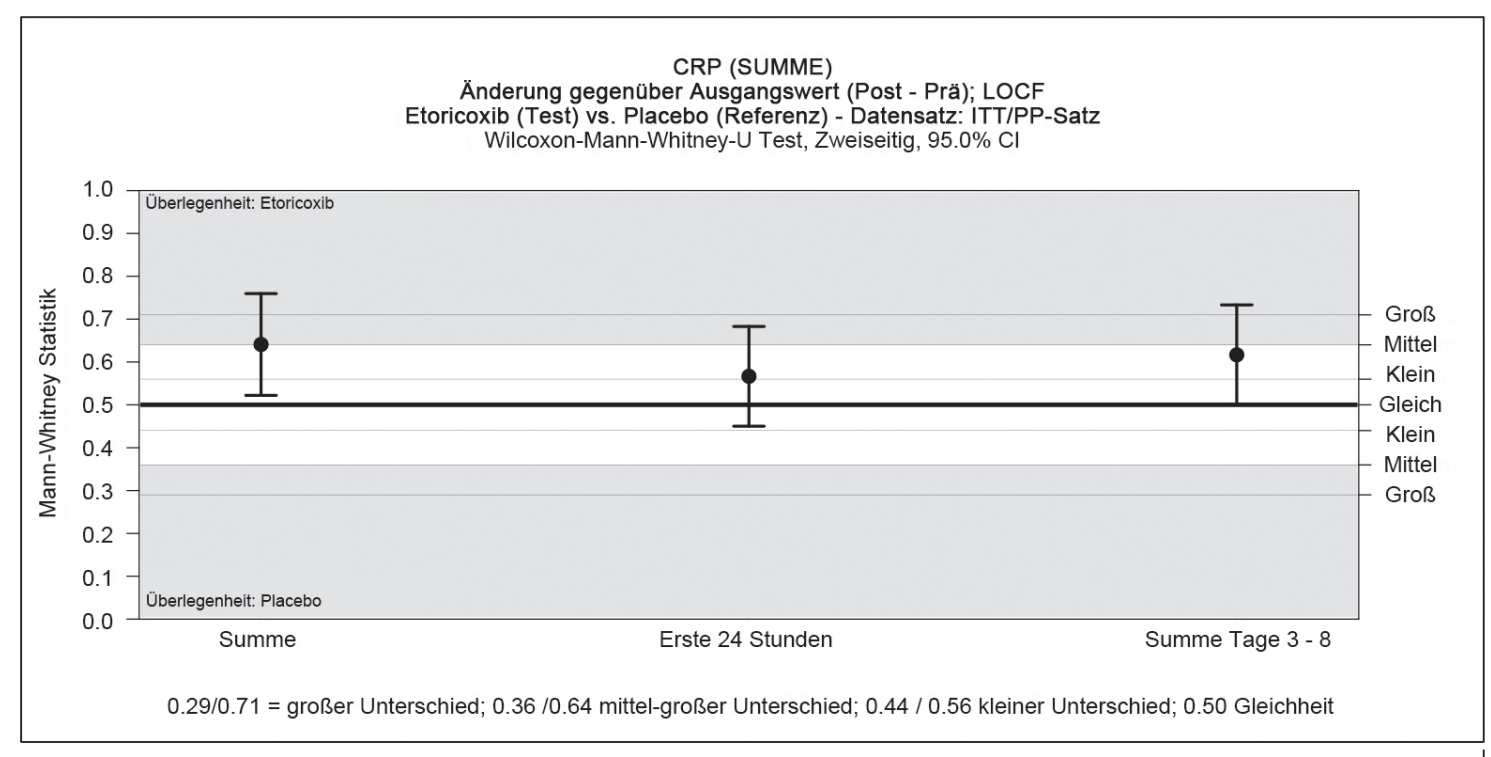

Abb. 11: Wilcoxon-Mann-Whitney Statistik - Vergleich der Behandlungsgruppen Etoricoxib vs. Placebo hinsichtlich hs CrP

\subsubsection{Leukozyten}

Nach der exzentrischen Belastung stieg die Zahl der Leukozyten im Blut als Marker für Entzündung an (siehe Tab. 15).

Tab. 15: Beschreibende Statistik - Änderung der Leukozytenanzahl gegenüber den Ausgangswerten (Phase 1 und 2)

\begin{tabular}{|l|l|l|l|}
\hline \multicolumn{2}{|l|}{ Änderung gegenüber Ausgangswerten } & Etoricoxib & Placebo \\
\hline N & 48 & 47 \\
\hline $\begin{array}{l}\text { Summe aller } \\
\text { Bewertungen }\end{array}$ & Mittelwert (SA) & $0,21(5,84)$ & $2,00(4,23)$ \\
\hline & Min-Median-Max & $-17,7-0,7-12,1$ & $-11,3-2,1-15,1$ \\
\hline Erste 24 Stunden & Mittelwert (SA) & $0,65(1,99)$ & $1,36(1,72)$ \\
\hline & Min-Median-Max & $-5,9-0,8-3,7$ & $-3,5-1,2-5,5$ \\
\hline Tag 3-Tag 8 & Mittelwert (SA) & $-0,44(4,25)$ & $0,64(2,94)$ \\
\hline & Min-Median-Max & $-15,7-0,45-8,6$ & $-8,3-0,8-9,6$ \\
\hline
\end{tabular}

In der crossover-Analyse konnte für die Etoricoxib-Gruppe ein statistisch nicht signifikanter Trend zu einem verminderten Leukozytenanstieg im Vergleich zur 
Placebo-Gruppe demonstriert werden ( $p=0,0981)$. Es zeigte sich kein Übertragungseffekt (siehe Tab. 16).

Tab. 16: Two-period crossover-Analyse - Vergleich der Behandlungsgruppen Etoricoxib vs. Placebo hinsichtlich der Leukozytenanzahl

Test Resultate

Übertragung

Behandlung

Phase

Wilcoxon T

(Sequenz A-B / B-A) $\quad 580,0$ / 596,0 $\quad 592,5$ / 583,5 522,0 / 654, 0

Asymptotisch unkorrigiert $\quad(\mathrm{DF}=1)$

$\begin{array}{llll}X^{2} & 0,0272 & 0,0086 & 1,8531 \\ \text { P-Wert } & 0,8690 & 0,9260 & 0,1734 \\ \text { er P-Wert } & 0,8742 & 0,9308 & 0,1767\end{array}$

Geschätzter Korrelationskoeffizient (Rho): 0,82

Test für Behandlungseffekt nur in Phase 1:

Asymp. P-Wert $=0,7649\left(\mathrm{X}^{2}=0,0894, \mathrm{DF}=1\right)$

Exakter P-Wert $=0,7709$

\subsubsection{Sedimentationsrate}

Die Sedimentationsrate zeigte nach der exzentrischen Belastung in beiden Behandlungsgruppen keine Veränderungen (siehe Tab. 17). Demzufolge konnten keine statistisch signifikanten Behandlungseffekte demonstriert werden (siehe Tab. 18).

Tab. 17: Beschreibende Statistik - Änderung der Sedimentationsrate gegenüber den Ausgangswerten (Phase 1 und 2)

\begin{tabular}{|l|l|l|l|}
\hline \multicolumn{2}{|l|}{ Änderung gegenüber Ausgangswerten } & Etoricoxib & Placebo \\
\hline $\mathrm{N}$ & 48 & 47 \\
\hline $\begin{array}{l}\text { Summe aller } \\
\text { Bewertungen }\end{array}$ & Mittelwert (SA) & $-2,0(18,35)$ & $-1,2(18,46)$ \\
\hline & Min-Median-Max & $-69,0-0,0-31,0$ & $-96,0-0,0-31,0$ \\
\hline
\end{tabular}




\begin{tabular}{|l|l|l|l|}
\hline \multicolumn{2}{|l|}{ Änderung gegenüber Ausgangswerten } & Etoricoxib & Placebo \\
\hline Erste 24 Stunden & Mittelwert (SA) & $0,5(6,38)$ & $-0,7(7,75)$ \\
\hline & Min-Median-Max & $-18,0-0,0-16,0$ & $-41,0-0,0-10,0$ \\
\hline Tag 3-Tag 8 & Mittelwert (SA) & $-2,5(13,63)$ & $-0,5(11,74)$ \\
\hline & Min-Median-Max & $-58,0-0,0-21,0$ & $-55,0-0,0-23,0$ \\
\hline
\end{tabular}

Tab. 18: Two-period crossover-Analyse - Vergleich der Behandlungsgruppen Etoricoxib vs. Placebo hinsichtlich der Sedimentationsrate

Test Resultate Übertragung Behandlung Phase

Wilcoxon T

(Sequenz A-B / B-A) $\quad 581,0 / 595,0 \quad 629,0 / 574,0 \quad 563,0 / 613,0$

Asymptotisch unkorrigiert $\quad(\mathrm{DF}=1)$

$\begin{array}{llll}X^{2} & 0,0208 & 0,7167 & 0,2661 \\ \text { P-Wert } & 0,8852 & 0,3972 & 0,6059 \\ \text { er P-Wert } & 0,8903 & 0,4036 & 0,6126\end{array}$

Geschätzter Korrelationskoeffizient (Rho): 0,70

Test für Behandlungseffekt nur in Segment 1:

Asymp. P-Wert $=0,6649\left(X^{2}=0,1876, D F=1\right)$

Exakter P-Wert $=0,6714$

\section{3 $\quad \mathrm{H}_{3}$ : Alter und Muskelschmerz nach exzentrischer Belastung}

Im Folgenden werden die Ergebnisse zur Prüfung der Arbeitshypothese $\mathrm{H}_{3}$ dargestellt: Es gibt hinsichtlich des Alters keinen Unterschied zwischen der Wirksamkeit von Etoricoxib und Placebo auf Muskelschmerz, auf die Erholung der Muskelkraft und auf Entzündungsmarker im Blut nach exzentrischer Belastung. 
Eine Analyse der Wirksamkeit von Etoricoxib im Vergleich zu Placebo in den Subgruppen „Jüngere“ und „Ältere“ wurde geplant, aufgrund der fehlenden statistisch signifikanten Behandlungseffekte in der Gesamtanalyse jedoch nicht durchgeführt. Gleichwohl wurden physiologisch relevante altersbezogene Unterschiede beobachtet. Um die altersbezogenen und nicht die behandlungsbezogenen Unterschiede zu untersuchen, wurde die Analyse der Subgruppen „Jüngere“ und „Ältere“ nur in Bezug auf die Placebo-Gruppe durchgeführt (siehe Tab. 19).

Tab. 19: Beschreibende Statistik - Ausgangswerte der Placebo-Subgruppen Jüngere und Ältere

\begin{tabular}{|c|c|c|c|}
\hline & & Jüngere & Ältere \\
\hline \multicolumn{2}{|l|}{$\mathrm{N}$} & 23 & 25 \\
\hline \multirow{3}{*}{$\begin{array}{l}\text { Bewegungs- } \\
\text { schmerz }\end{array}$} & Mittelwert (SA) & $7,0(1,49)$ & $6,3(1,52)$ \\
\hline & Min-Median-Max & $3-7-9$ & $3-6-8$ \\
\hline & P-Wert & \multicolumn{2}{|l|}{0,1549} \\
\hline \multirow[t]{3}{*}{ Ruheschmerz } & Mittelwert (SA) & $2,9(2,01)$ & $2,0(1,58)$ \\
\hline & Min-Median-Max & $0-3-6$ & $0-2-5$ \\
\hline & P-Wert & \multicolumn{2}{|l|}{0,1253} \\
\hline \multirow[t]{3}{*}{ Maximalkraft [kg] } & Mittelwert (SA) & $51,04(15,74)$ & $51,95(27,98)$ \\
\hline & Min-Median-Max & $21,8-57,6-77,2$ & $22,5-45,0-150,2$ \\
\hline & P-Wert & \multicolumn{2}{|l|}{0,4703} \\
\hline \multirow{3}{*}{$\begin{array}{l}\text { Schmerzschwelle } \\
\text { am max. Druck- } \\
\text { punkt }\left[{ }^{\circ} \mathrm{C}\right]\end{array}$} & Mittelwert (SA) & $48,45(1,91)$ & $49,73(1,38)$ \\
\hline & Min-Median-Max & $42,2-48,5-51,2$ & $46,8-50,0-52,0$ \\
\hline & P-Wert & \multicolumn{2}{|l|}{0,0147} \\
\hline $\mathrm{CrP}$ & Mittelwert (SA) & $0,0832(0,069)$ & $0,1266(0,181)$ \\
\hline
\end{tabular}




\begin{tabular}{|l|l|l|l|}
\hline \multicolumn{2}{|l|}{} & Jüngere & Ältere \\
\hline & Min-Median-Max & $\begin{array}{l}0,017-0,057- \\
0,343\end{array}$ & $\begin{array}{l}0,026-0,087- \\
0,131\end{array}$ \\
\hline & P-Wert & 0,1800 & \multicolumn{2}{l|}{} \\
\hline Leukozyten & Mittelwert (SA) & $6,27(1,76)$ & $3,9-5,6-8,1$ \\
\hline & Min-Median-Max & $4,1-5,9-10,5$ & \multicolumn{2}{l|}{} \\
\hline $\begin{array}{l}\text { Sedimentationsra- } \\
\text { te }\end{array}$ & P-Wert & 0,5839 & $7,6(8,3)$ \\
\hline & Mittelwert (SA) & $3,7(2,85)$ & 2-2-42 \\
\hline & Min-Median-Max & $\mathbf{2 - 2 - 1 1}$ & \multicolumn{2}{|l}{} \\
\hline & P-Wert & $\mathbf{0 , 0 1 0 5}$ & \multicolumn{2}{l}{} \\
\hline
\end{tabular}

\subsubsection{Schmerz}

Die Homogenitätsanalyse der Ausgangswerte für Ruheschmerz, Bewegungsschmerz und Schmerzschwelle am max. Druckpunkt innerhalb der PlaceboGruppe demonstrierte deutliche, teilweise statistisch signifikante Unterschiede zwischen den jüngeren und älteren Erwachsenen (siehe Abb. 12).

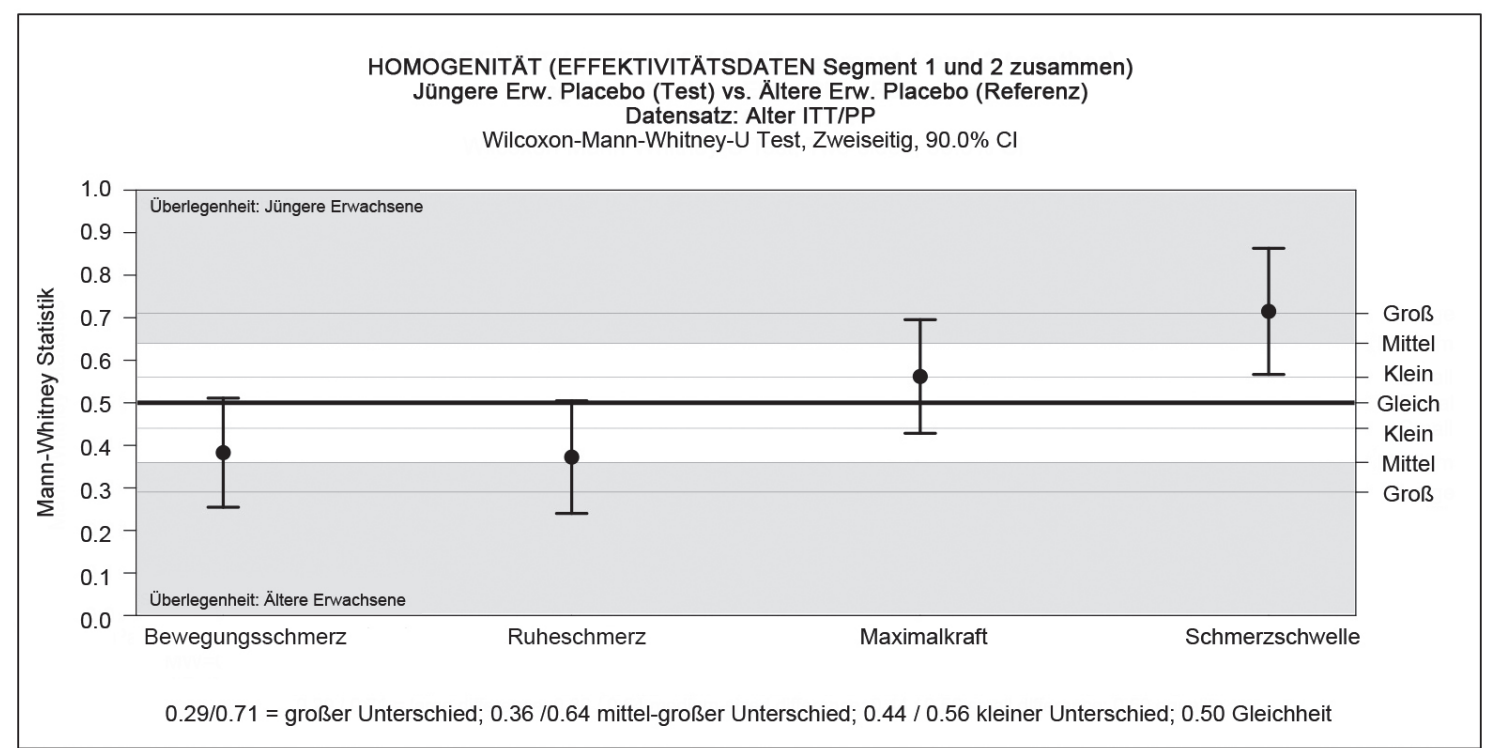

Abb. 12: Wilcoxon-Mann-Whitney Statistik - Homogenitätsanalyse der Ausgangswerte für Schmerz und Maximalkraft der Placebo-Subgruppen Jüngere und Ältere 


\subsubsection{Ruheschmerz}

In der Placebo-Gruppe zeigten die Jüngeren (Mittelwert: 2,9 \pm 2,01) im Vergleich zu den Älteren (Mittelwert: $2,0 \pm 1,58$ ) ausgangs statistisch nicht signifikant höhere Werte für Schmerz in Ruhe nach exzentrischer Belastung ( $p=$ 0,1253) (siehe Tab. 19 sowie Abb. 12).

Im Verlauf verringerten sich die Ruheschmerzen der Jüngeren statistisch signifikant schneller als die der Älteren (Summe: $p=0,0160$; Tag 3-8: $p=0,0080$ ) (siehe Tab. 20 sowie Abb. 13).

Tab. 20: Beschreibende Statistik - Änderung gegenüber den Ausgangswerten für Ruheschmerz der Placebo-Subgruppen Jüngere und Ältere (Phase 1 und 2)

\begin{tabular}{|l|l|l|l|}
\hline \multicolumn{2}{|l|}{ Änderung gegenüber Ausgangswerten } & Jüngere & Ältere \\
\hline N & 23 & 24 \\
\hline $\begin{array}{l}\text { Summe aller Be- } \\
\text { wertungen }\end{array}$ & Mittelwert (SA) & $-16,5(12,6)$ & $-6,2(13,7)$ \\
\hline & Min-Median-Max & $-37,0-(-20,0)-7,0$ & $-29,0-(-6,5)-$ \\
& P-Wert & $\mathbf{0 , 0 1 6 0}$ & 35,0 \\
\hline & Mittelwert (SA) & $-0,2(3,4)$ & $-0,9(4,0)$ \\
\hline Erste 24 Stunden & Min-Median-Max & $-7,0-0,0-6,0$ & $-7,0-0,5-11,0$ \\
\hline & P-Wert & 0,3190 & \multicolumn{2}{l|}{} \\
\hline Tag 3-Tag 8 & Mittelwert (SA) & $-16,1(10,4)$ & $-7,1(10,5)$ \\
\hline & Min-Median-Max & $-32,0-(-18,0)-2,0$ & $-23,0-(-7,5)-$ \\
& & \multicolumn{2}{l}{} \\
\hline & P-Wert & $\mathbf{0 , 0 0 8 0}$ & \multicolumn{2}{l|}{} \\
\hline
\end{tabular}




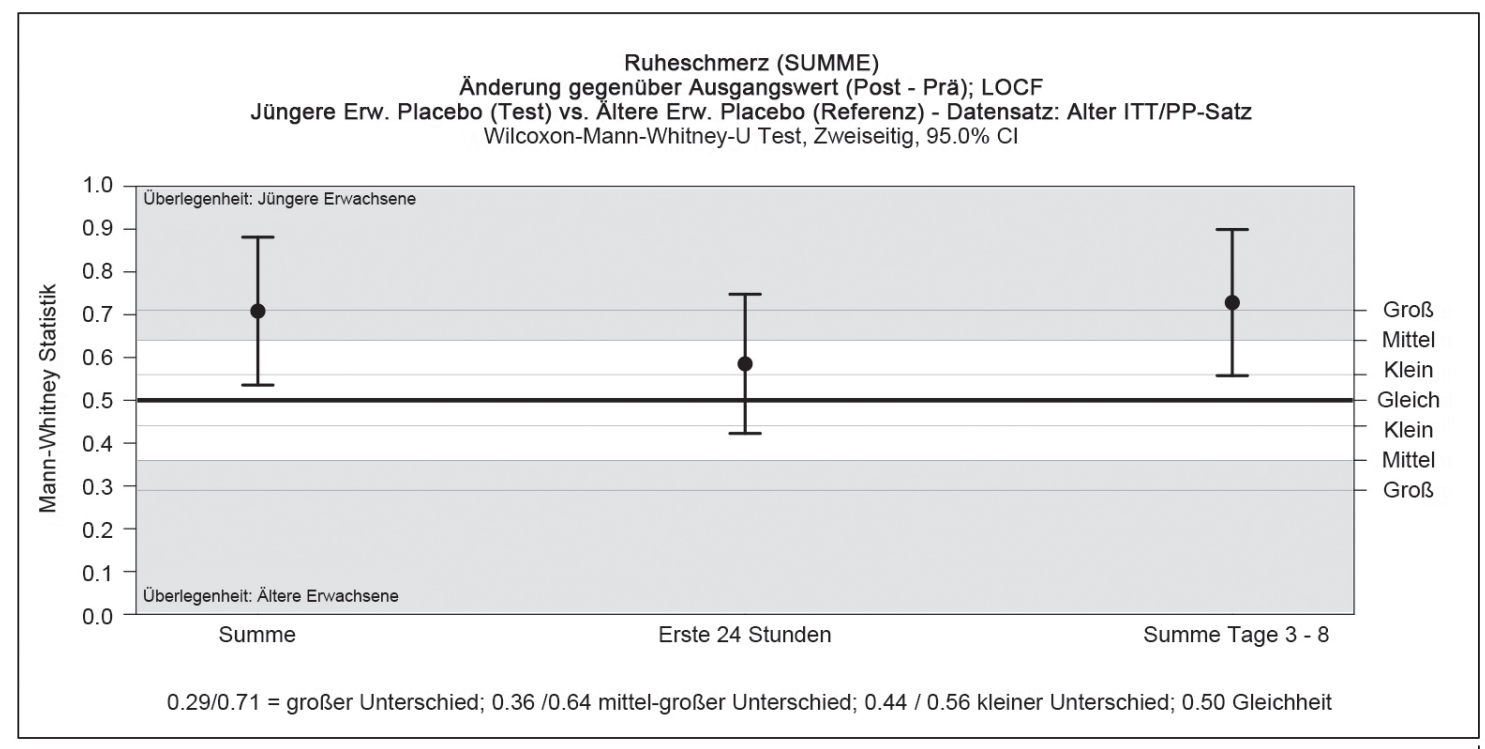

Abb. 13: Wilcoxon-Mann-Whitney Statistik - Vergleich der Placebo-Subgruppen Jüngere und Ältere hinsichtlich der Änderung gegenüber den Ausgangswerten von Ruheschmerz

\subsubsection{Bewegungsschmerz}

In der Placebo-Gruppe zeigten die Jüngeren (Mittelwert: 7,0 $\pm 1,49$ ) im Vergleich zu den Älteren (Mittelwert: 6,3 $\pm 1,52$ ) ausgangs statistisch nicht signifikant höhere Werte für Bewegungsschmerz nach exzentrischer Belastung ( $p=$ 0,1549) (siehe Tab. 19 sowie Abb. 12).

Im Verlauf zeigten sich keine statistisch signifikanten Unterschiede zwischen der Gruppe der Jüngeren und der Gruppe der Älteren (siehe Tab. 21).

Tab. 21: Beschreibende Statistik - Änderung gegenüber den Ausgangswerten für Bewegungsschmerz der Placebo-Subgruppen Jüngere und Ältere (Phase 1 und 2)

\begin{tabular}{|l|l|l|l|}
\hline \multicolumn{2}{|l|}{ Änderung gegenüber Ausgangswerten } & Jüngere & Ältere \\
\hline N & 23 & 24 \\
\hline $\begin{array}{l}\text { Summe aller } \\
\text { Bewertungen }\end{array}$ & Mittelwert (SA) & $-17,5(13,3)$ & $-15,8(13,3)$ \\
\hline & Min-Median-Max & $-44,0-(-18,0)-11,0$ & $\begin{array}{l}-33,0-(-19,5)- \\
25,0\end{array}$ \\
\hline & P-Wert & 0,8791 & $-0,6(4,2)$ \\
\hline Erste 24 Stunden & Mittelwert (SA) & $-0,1(3,2)$ & \multicolumn{2}{l}{} \\
\hline
\end{tabular}




\begin{tabular}{|l|l|l|l|}
\hline \multicolumn{2}{|l|}{ Änderung gegenüber Ausgangswerten } & Jüngere & Ältere \\
\hline & Min-Median-Max & $-9,0-0,0-6,0$ & $-8,0-0,0-9,0$ \\
\hline & P-Wert & 0,6148 & \\
\hline Tag 3-Tag 8 & Mittelwert (SA) & $-17,0(11,2)$ & $-15,2(10,2)$ \\
\hline & Min-Median-Max & $-35,0-(-20,0)-7,0$ & $-29,0-(-18,0)-$ \\
& & & 16,0 \\
\hline & P-Wert & 0,6477 & \\
\hline
\end{tabular}

\subsubsection{Schmerzschwelle am max. Druckpunkt}

In der Placebo-Gruppe zeigten die Jüngeren (Mittelwert: 48,45 $\pm 1,91$ ) im Vergleich zu den Älteren (Mittelwert: 49,73 \pm 1,38) ausgangs eine statistisch signifikant niedrigere Schmerzschwelle am max. Druckpunkt nach exzentrischer Belastung ( $p=0,0147)$ (siehe Tab. 19 sowie Abb. 12).

Im Verlauf zeigten sich keine statistisch signifikanten Unterschiede zwischen der Gruppe der Jüngeren und der Gruppe der Älteren (siehe Tab. 22).

Tabelle 22: Beschreibende Statistik - Änderung gegenüber den Ausgangswerten der Schmerzschwelle am max. Druckpunkt der Placebo-Subgruppen Jüngere und Ältere (Phase 1 und 2)

\begin{tabular}{|l|l|l|l|}
\hline \multicolumn{2}{|l|}{ Änderung gegenüber Ausgangswerten } & Jüngere & Ältere \\
\hline N & 19 & 24 \\
\hline $\begin{array}{l}\text { Summe aller } \\
\text { Bewertungen }\end{array}$ & Mittelwert (SA) & $3,8(6,1)$ & $2,7(4,7)$ \\
\hline & Min-Median-Max & $-3,4-3,3-21,0$ & $-9,3-2,2-15,4$ \\
\hline & P-Wert & 0,7692 & \multicolumn{2}{l|}{} \\
\hline Erste 24 Stunden & Mittelwert (SA) & $0,5(2,9)$ & $0,5(2,0)$ \\
\hline & Min-Median-Max & $-4,2-0,4-9,0$ & $-5,1-0,4-5,2$ \\
\hline & P-Wert & 0,6848 & $2,2(2,9)$ \\
\hline Tag 3-Tag 8 & Mittelwert (SA) & $3,0(3,2)$ & $-4,2-1,9-10,2$ \\
\hline & Min-Median-Max & $-0,3-2,4-12,0$ & \multicolumn{2}{l|}{} \\
\hline
\end{tabular}




\begin{tabular}{|l|l|l|l|}
\hline \multicolumn{2}{|l|}{ Änderung gegenüber Ausgangswerten } & Jüngere & Ältere \\
\hline & P-Wert & 0,5073 & \\
\hline
\end{tabular}

\subsubsection{Maximalkraft}

Die Ausgangswerte der Maximalkraft waren zwischen den beiden Altersgruppen vergleichbar (siehe Tab. 19 sowie Abb. 12).

Im Verlauf zeigte sich ein statistisch nicht signifikanter Trend zur schnelleren Wiederherstellung der Maximalkraft in der Gruppe der Jüngeren im Vergleich zur Gruppe der Älteren (Summe: $p=0,1303$; Tage 3-8: $p=0.0584$ ) (siehe Tab. 23 sowie Abb. 14).

Tab. 23: Beschreibende Statistik - Änderung gegenüber den Ausgangswerten der Maximalkraft der Placebo-Subgruppen Jüngere und Ältere (Phase 1 und 2)

\begin{tabular}{|l|l|l|l|}
\hline \multicolumn{2}{|l|}{ Änderung gegenüber Ausgangswerten } & Jüngere & Ältere \\
\hline N & & 23 & 24 \\
\hline $\begin{array}{l}\text { Summe aller Be- } \\
\text { wertungen }\end{array}$ & Mittelwert (SA) & $42,7(78,9)$ & $15,0(57,2)$ \\
\hline & Min-Median-Max & $-45,2-17,4-311,2$ & $-85,6-1,8-154,2$ \\
\hline & P-Wert & 0,1303 & \\
\hline Erste 24 Stunden & Mittelwert (SA) & $1,9(29,4)$ & $0,4(23,2)$ \\
\hline & Min-Median-Max & $-61,0-0,4-111,3$ & $-50,5-(-4,3)-$ \\
& P-Wert & 0,6809 & 70,4 \\
\hline Tag 3-Tag 8 & Mittelwert (SA) & $40,8(56,2)$ & $14,7(38,2)$ \\
\hline & Min-Median-Max & $-17,7-17,8-199,9$ & $-36,1-5,2-115,9$ \\
\hline & P-Wert & 0,0584 & \\
\hline
\end{tabular}




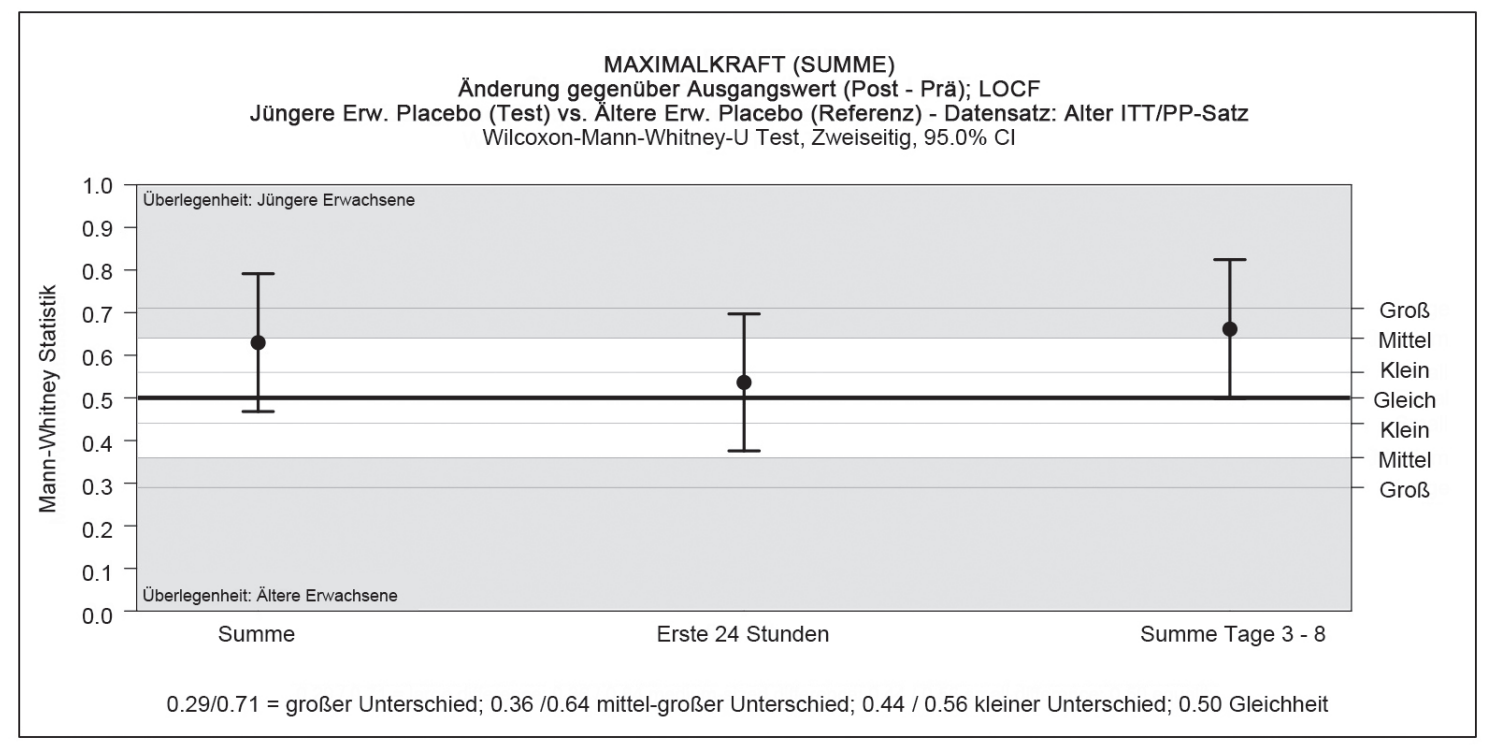

Abb. 14: Wilcoxon-Mann-Whitney Statistik - Vergleich der Placebo-Subgruppen Jüngere und Ältere hinsichtlich der Maximalkraft

\subsubsection{Entzündungsmarker}

Die Homogenitätsanalyse der Ausgangswerte für CrP und Sedimentationsrate innerhalb der Placebo-Gruppe demonstrierte deutliche, teilweise statistisch signifikante Unterschiede zwischen den jüngeren und älteren Erwachsenen (siehe Abb. 15).

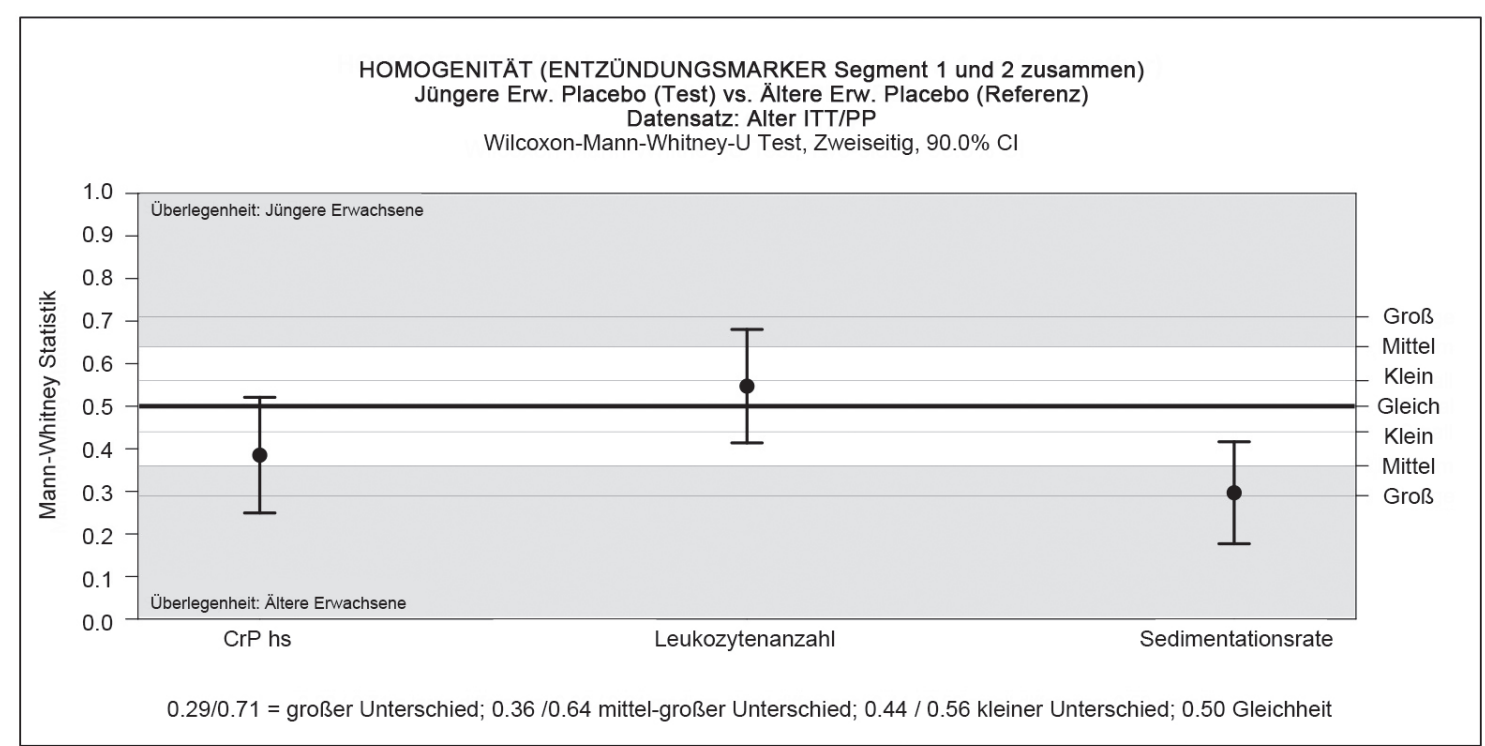

Abb. 15: Wilcoxon-Mann-Whitney Statistik - Homogenitätsanalyse der Ausgangswerte für Entzündungsmarker der Placebo-Subgruppen Jüngere und Ältere 


\subsubsection{CrP}

Die Ausgangswerte für CrP waren in der Gruppe der Älteren im Vergleich nicht statistisch signifikant erhöht $(p=0,1800)$ (siehe Abb. 15).

Im Verlauf zeigten sich keine altersbedingten Unterschiede für CrP (siehe Tab. 24).

Tab. 24: Beschreibende Statistik - Änderung gegenüber den Ausgangswerten für CrP der Placebo-Subgruppen Jüngere und Ältere (Phase 1 und 2)

\begin{tabular}{|l|l|l|l|}
\hline \multicolumn{2}{|l|}{ Änderung gegenüber Ausgangswerten } & Jüngere & Ältere \\
\hline N & 23 & 23 \\
\hline $\begin{array}{l}\text { Summe aller } \\
\text { Bewertungen }\end{array}$ & Mittelwert (SA) & $0,083(0,540)$ & $0,085(0,484)$ \\
\hline & Min-Median-Max & $\begin{array}{l}-0,673-(-0,002)- \\
2,387\end{array}$ & $\begin{array}{l}-0,493-0,008- \\
2.179\end{array}$ \\
\hline & P-Wert & 0,6516 & $-0,0212(0,080)$ \\
\hline Erste 24 Stunden & Mittelwert (SA) & $-0,0447(0,037)$ & $-0,336-(-0,002)-$ \\
\hline & Min-Median-Max & $\begin{array}{l}-0,068-(-0,01)- \\
0,104\end{array}$ & 0,047 \\
\hline & P-Wert & 0,4959 & $0,1062(0,541)$ \\
\hline Tag 3-Tag 8 & Mittelwert (SA) & $0,0877(0,542)$ & $-0,323-0,004-$ \\
\hline & Min-Median-Max & $\begin{array}{l}-0,74-(-0,011)- \\
2,397\end{array}$ \\
\hline & & 0,7318 & \multicolumn{2}{l}{} \\
\hline & P-Wert & \multicolumn{2}{l}{} \\
\hline
\end{tabular}

\subsubsection{Leukozyten}

Die Ausgangswerte der Leukozyten waren zwischen den beiden Altersgruppen vergleichbar (siehe Abb. 15).

Im Verlauf zeigten sich keine altersbedingten Unterschiede der Leukozytenanzahl (siehe Tab. 25). 
Tab. 25: Beschreibende Statistik - Änderung gegenüber den Ausgangswerten für Leukozyten der Placebo-Subgruppen Jüngere und Ältere (Phase 1 und 2)

\begin{tabular}{|l|l|l|l|}
\hline \multicolumn{2}{|l|}{ Änderung gegenüber Ausgangswerten } & Jüngere & Ältere \\
\hline N & 23 & 24 \\
\hline $\begin{array}{l}\text { Summe aller } \\
\text { Bewertungen }\end{array}$ & Mittelwert (SA) & $1,62(3,73)$ & $2,36(4,72)$ \\
\hline & Min-Median-Max & $-7,6-2,1-7,0$ & $-11,3-1,95-15,1$ \\
\hline & P-Wert & 0,8868 & \multicolumn{2}{l|}{} \\
\hline Erste 24 Stunden & Mittelwert (SA) & $1,12(1,70)$ & $1,59(1,75)$ \\
\hline & Min-Median-Max & $-3,5-1,0-3,8$ & $-3,0-1,35-5,5$ \\
\hline & P-Wert & 0,3889 & \multicolumn{2}{|l}{} \\
\hline Tag 3-Tag 8 & Mittelwert (SA) & $0,5(2,67)$ & $0,77(3,22)$ \\
\hline & Min-Median-Max & $-6,8-0,8-6,5$ & $-8,3-0,95-9,6$ \\
\hline & P-Wert & 0,6312 & \multicolumn{2}{l|}{} \\
\hline
\end{tabular}

\subsubsection{Sedimentationsrate}

Die Ausgangswerte der Sedimentationsrate waren in der Gruppe der Älteren im Vergleich statistisch signifikant erhöht $(p=0,0105)$ (siehe Abb. 15).

Im Verlauf zeigten sich keine altersbedingten Unterschiede der Sedimentationsrate (siehe Tab. 26).

Tab. 26: Beschreibende Statistik - Änderung gegenüber den Ausgangswerten der Sedimentationsrate der Placebo-Subgruppen Jüngere und Ältere (Phase 1 und 2)

\begin{tabular}{|l|l|l|l|}
\hline \multicolumn{2}{|l|}{ Änderung gegenüber Ausgangswerten } & Jüngere & Ältere \\
\hline N & 23 & 24 \\
\hline $\begin{array}{l}\text { Summe aller } \\
\text { Bewertungen }\end{array}$ & Mittelwert (SA) & $0,4(9,43)$ & $-2,7(24,3)$ \\
\hline & Min-Median-Max & $-18,0-0,0-24,0$ & $-96,0-0,0-31,0$ \\
\hline & P-Wert & 0,9703 & \\
\hline Erste 24 Stunden & Mittelwert (SA) & $-0,3(3,24)$ & $-1,1(10,47)$ \\
\hline & Min-Median-Max & $-10,0-0,0-4,0$ & $-1,5-0,0-10,0$ \\
\hline
\end{tabular}




\begin{tabular}{|l|l|l|l|}
\hline \multicolumn{2}{|l|}{ Änderung gegenüber Ausgangswerten } & Jüngere & \multicolumn{2}{l|}{ Ältere } \\
\hline & P-Wert & 0,7042 & $-1,5(14,73)$ \\
\hline Tag 3-Tag 8 & Mittelwert (SA) & $0,7(7,66)$ & $-55,0-0,0-22,0$ \\
\hline & Min-Median-Max & $-14,0-0,0-23,0$ & \multicolumn{2}{|l}{} \\
\hline & P-Wert & 0,9196 & \multicolumn{2}{|l|}{} \\
\hline
\end{tabular}




\section{DISKUSSION}

\section{$5.1 \quad$ Studienmodell}

In der Studie wurde die exzentrische Belastung der unteren Extremitäten am Trainingsgerät „extra-fit Beinstrecker" durchgeführt. Die Ergebnisse zeigen, dass durch das Belastungsmodell erfolgreich DOMS der Oberschenkelmuskulatur erzeugt werden kann. Das Auftreten von Muskelschmerz und die Abnahme der Muskelkraft nach der exzentrischen Belastung in jeweils statistisch signifikanter Ausprägung zeigten einen ähnlich wie in vorhergehenden Studien beschriebenen typischen zeitlichen Verlauf von DOMS (siehe Abb. 5, 7 und 9). In der Studie entstand nach der exzentrischen Belastung ein Muskelschmerz, der innerhalb der ersten 40 Stunden am ausgeprägtesten war und über einen Zeitraum von ca. 6 Tagen anhielt. Zudem wurde ein Muskelkraftverlust von bis zu $30 \%$ beobachtet, der ebenfalls innerhalb der ersten 40 Stunden am ausgeprägtesten war und über einen Zeitraum von 4-7 Tagen bestand. Diese Ergebnisse zeigen, dass das Belastungsmodell mit anderen Modellen, die bisher zur Erzeugung von DOMS eingesetzt wurden, vergleichbar ist.

In vorhergehenden Studien wurde eine Reihe von verschiedenen exzentrischen Belastungsmodellen verwendet. Es kamen sowohl apparative als auch nicht apparative Modelle zur Anwendung. Nicht-gerätebezogene Modelle sind praxisrelevant und bilden die alltägliche Belastung der Muskulatur sehr gut ab (Rother et al. 2011). In Modellen wie z. B. „Bergablaufen“ (Donnelly et al. 1988, Donnelly et al. 1990) und "Sprünge in die Tiefe" (Semark et al. 1999) werden Bewegungen durchgeführt, die erhebliche exzentrische Belastungen beinhalten und mehr oder weniger alltägliche Bewegungsabläufe darstellen. Ein Nachteil dieser Modelle ist die Schwierigkeit, die Belastung zu standardisieren. Jeder Proband hat bei Bergabläufen einen individuellen Laufstil (eine unterschiedliche Schrittlänge, Abrollphase, etc.) und muss ein individuelles, im Vergleich zu anderen Probanden meist abweichendes Körpergewicht, bewegen. Hinzu 
kommen weitere schwer zu beeinflussende äußere Faktoren (wie z. B. Unebenheiten des Untergrundes). In der Summe erschweren diese Unterschiede bei Untersuchungsdurchführung und Untersuchungsbedingungen eine Standardisierung des Belastungsmodells. Eine mangelnde Standardisierung des Modells bedeutet, dass erhebliche interindividuelle Unterschiede im Ausmaß der exzentrischen Belastung und damit letztendlich auch mögliche Heterogenitäten in der Ausprägung von DOMS auftreten können. Das kann entsprechende Einschränkungen in der Validität der Studienergebnisse bedeuten.

Das vermehrt in neueren Studien eingesetzte Modell „Treppabsteigen“ ist ein besser zu standardisierendes, nicht-gerätebezogenes Modell zur Erzeugung von DOMS (Rother et al. 2011, Seidel et al. 2012). Kritische Faktoren, wie z. B. die Einheitlichkeit in Laufstil und Laufgeschwindigkeit können in vorhergehenden Übungseinheiten angepasst, eingeübt, durch Studienpersonal überwacht und somit standardisiert werden. Unterschiede im Körpergewicht können durch eine Anpassung der Wegstrecke an das jeweilige Körpergewicht ausgeglichen werden. Dieses Modell berücksichtigt zwar die Alltagsrelevanz der Belastungsform und gewisse individuelle Unterschiede zwischen den Probanden, es ermöglicht jedoch ebenfalls keine vollständige Standardisierung der exzentrischen Belastung. Das Ausmaß der Belastung wird für jeden Probanden anhand des Körpergewichtes nur geschätzt. Es wird lediglich angenommen, welche Leistung ein Proband - bezogen auf sein Körpergewicht - theoretisch erbringen sollte. Ein Einfluss von Konstitution, Muskel- und Fettanteil sowie weiteren individuellen Faktoren, die die Leistungsfähigkeit beeinflussen, wird durch dieses Modell nicht berücksichtigt. Dadurch könnten auch hier Heterogentitäten in der Ausprägung des erzeugten Muskelkaters entstehen. Eine weitere Einschränkung dieses Modells zeigte sich für Studien mit Crossover-Design. Rother et al. beobachteten in ihrer Studie zu Celecoxib das Auftreten eines RBEs, bedingt durch die wiederholte Durchführung des Treppabsteigens in Phase 2 der Studie. Folglich musste die Validität der 
Studienergebnisse in Frage gestellt werden. Aussagekräftige Ergebnisse könnten mit diesem Modell lediglich in einem Parallelgruppen-Design erzielt werden (Rother et al. 2011).

Demgegenüber sind gerätebezogene Modelle, wie z. B. die häufig angewandten Ellenbogenflexor-Modelle (Rother et al. 2009) oder das in dieser Studie verwendete Kniestrecker-Modell gut standardisierbar. Zwar reflektieren sie nicht unbedingt Belastungen, wie sie im alltäglichen Leben auftreten. Am Beispiel unseres Studienmodells wird jedoch deutlich, dass der Gerätebezug eine nahezu vollständige Standardisierung der Belastung ermöglicht. Durch die Möglichkeit die Sitzposition individuell an Größe und Konstitution der Probanden anzupassen und durch den vom Gerät vorgegebenen Bewegungsspielraum ist die exzentrische Belastung einer gezielten Muskelgruppe (Kniestrecker) möglich. Der Ablauf und die Geschwindigkeit des Bewegungszyklus wird in einer Übungsseinheit erlernt und durch geschultes Studienpersonal überwacht und korrigiert, sodass jeder Proband exakt die gleiche Bewegung ausführt. Das Belastungsgewicht wird nicht in Bezug auf das individuelle Körpergewicht, sondern in Bezug auf die individuelle Muskelkraft für jeden Probanden genau festgelegt. Durch die exakte Anpassung der Belastung - auch unter Berücksichtigung von konstitutions-, geschlechts- und altersspezifischen individuellen Muskelverhältnissen, Trainingszuständen und Hebelverhältnissen - an die individuelle maximale Muskelkraft, ist die Erzeugung von DOMS in ähnlich starker Ausprägung bei allen Probanden möglich und somit in hohem Maße standardisiert und homogenisiert.

Zusätzlich zu der Möglichkeit, die exzentrische Belastung interindividuell anzugleichen, ist es möglich, die Kraftdifferenzen zwischen dominantem und nicht dominatem Bein jedes Probanden zu berücksichtigen. Durch die Belastung von nur einem Bein während Phase 1 und dem anderen Bein während Phase 2 wird die wiederholte exzentrische Belastung ein- und derselben Muskelgruppe umgangen und das Auftreten eines RBEs vermieden. Somit wird die Durchführung einer Studie im Crossover-Design ermöglicht. Die Robustheit dieser Herange- 
hensweise wurde in dieser Studie durch die fehlenden, statistisch signifikanten Übertragungseffekte in den Auswertungen von Muskelschmerz und Maximalkraft bestätigt.

Das Modell offenbarte jedoch Mängel bei der Methode zur Bestimmung des Bewegungsschmerzes. Der in dieser Studie beschriebene zeitliche Verlauf des Bewegungsschmerzes weicht von dem in vorherigen Studien ab. Maximale Werte für Bewegungsschmerz (NRS 6,5-7) wurden innerhalb der ersten 24 Stunden nach Behandlungsbeginn (entsprechend der ersten 40 Stunden nach exzentrischer Belastung) beobachtet. Die Werte nahmen bis 120 Stunden nach Belastung kontinuierlich ab und pendelten sich bei Werten um 3-3,5 ein. Im Gegensatz zu vorherigen Studien (Rother et al. 2011), normalisierten sich die Werte in dieser Studie jedoch nicht bis zum Ende des Nachbeobachtungszeitraums von 8 Tagen und es wurden zu keiner Zeit Werte um 0 erreicht. Dieses kann durch die Methode erklärt werden, die zur Bestimmung des Bewegungsschmerzes angewandt wurde. In der Nachbeobachtungsphase der Studie wurden die Bestimmung von Bewegungsschmerz und die Bestimmung der maximalen Muskelkraft gleichzeitig durchgeführt. Während die Probanden also bis zur vollständigen Erschöpfung der Oberschenkelmuskulatur das Gewicht während der Maximalkraftbestimmung bewegten, sollten sie gleichzeitig ihren Muskelschmerz während dieser konzentrischen Kontraktionen bewerten (Bestimmung von Bewegungsschmerz). Die während maximaler Kraftanstrengung angegebenen leichten Schmerzen im Oberschenkel (NRS 3-3,5) - auch außerhalb des Zeitraums, in dem typischerweise ein Bestehen von DOMS in der Literatur beschrieben wird (Clarkson und Hubal 2002) - reflektieren eher die große Anstrengung der Probanden, während der Maximalkraftbestimmung den Vorgaben der Studien gerecht zu werden und sind weniger hinweisend auf das Vorliegen von Muskelschmerzen im Rahmen von DOMS. Aus diesem Grund ist es nachvollziehbar, dass die Bewertungen von Bewegungsschmerz zu keiner Zeit Werte um 0 erreichten. Die Ergebnisse für Bewegungsschmerz sind somit aufgrund dieses Methodikfehlers nicht verwertbar. 
In der Studie wurde zudem eine alternative Methode zur Schmerzbewertung nach exzentrischer Belastung eingesetzt. Die Applikationen von Hitzestimuli mit einer Thermode zur Evaluierung der Schmerzempfindlichkeit des Muskels auf physikalische Reize nach exzentrischer Belastung wurde in anderen Studien zu DOMS bisher nicht angewandt. Aufgrund der in ähnlichen Studien gezeigten Möglichkeit durch dieses Verfahren Schmerzereignisse noch objektiver zu bewerten (Rother und Rother 2011), wurde die Methode in dieser Studie verwendet. Die Durchführung dieser Methode war für Untersucher und Probanden gleichermaßen einfach reproduzierbar. Die Robustheit dieser Methode wurde durch die Übereinstimmung der erzielten Ergebnisse mit denen der NRS-Methode bestätigt. Nach exzentrischer Belastung zeigte sich eine insgesamt reduzierte Hitzeschmerzschwelle (siehe Abb. 8) und eine im Vergleich zwischen jüngeren und älteren Probanden unterschiedlich starke Reduktion der Schmerzschwelle (siehe Abb. 12). Die Ergebnisse zeigen, dass die Methode der Hitzeschmerzschwelle ein geeignetes Mittel ist, das Ausmaß der durch das Modell erzeugten Muskelschmerzen objektiv darzustellen.

Der in vorhergehenden Publikationen beschriebene Anstieg der Entzündungsparameter während DOMS (Malm et al. 2000) konnte in dieser Studie bestätigt werden. Die verschiedenen gemessenen Entzündungsmarker reagierten jedoch nicht in gleichem Maße auf die exzentrische Belastung. In diesem Modell erwies sich hs CrP als sensitivster der gemessenen Entzündungsparameter (siehe Abb. 11). Die Anzahl der Leukozyten zeigte einen leichten Anstieg nach Belastung. Die Werte der Sedimentationsrate wurden durch die exzentrische Belastung in diesem Modell hingegen überhaupt nicht beeinflusst. Somit scheint der Parameter hs CrP für das Modell am geeignetsten zu sein, die Entzündungsreaktion nach exzentrischer Belastung darzustellen.

\section{2 $H_{1}$ : Etoricoxib bei Muskelschmerz nach exzentrischer Belastung}

Im folgenden Abschnitt soll die Arbeitshypothese $\mathrm{H}_{1}$ diskutiert werden: Es gibt keinen Unterschied zwischen der Wirksamkeit von Etoricoxib und Placebo auf 
Muskelschmerz, auf die Erholung der Muskelkraft und auf Entzündungsmarker im Blut nach exzentrischer Belastung.

In der Studie konnte insgesamt kein statistisch signifikanter Behandlungseffekt für Etoricoxib bei Muskelschmerz nach exzentrischer Belastung nachgewiesen werden. Die Summe aller abgegebenen Bewertungen für Ruheschmerz als auch für Bewegungsschmerz zeigten keine statistisch signifikanten Unterschiede zwischen den Behandlungsgruppen (siehe Abb. 3 und 4). Bei Betrachtung verschiedener zeitlicher Phasen innerhalb des Nachbeobachtungszeitraums konnten gleichwohl Unterschiede zwischen den Behandlungsgruppen demonstriert werden. Bei der separaten Untersuchung der Frühphase (erste 24 Stunden) nach exzentrischer Belastung konnte ein Trend zur Schmerzreduktion unter der Behandlung mit Etoricoxib festgestellt werden. Innerhalb der ersten 24 Stunden nach exzentrischer Belastung wurde eine statistisch nicht signifikante Reduktion des Ruheschmerzes ( $p=0,1341)$ unter Etoricoxib-Einnahme beobachtet (siehe Abb. 6). Dieses Ergebnis spricht für eine leicht positive Wirksamkeit von Etoricoxib auf das Erleben von Muskelschmerz im Rahmen von DOMS innerhalb der ersten Stunden nach exzentrischer Belastung. Etoricoxib scheint im Vergleich zu Placebo den Muskelschmerz in der Frühphase von DOMS leicht abzuschwächen.

Bei der Betrachtung der Ergebnisse über den gesamten Nachbeobachtungszeitraum von 168 Stunden konnte kein Trend zur Schmerzreduktion gezeigt werden. Insgesamt und insbesondere in der späteren Beobachtungsphase (nach 24 Stunden) nach exzentrischer Belastung scheint Etoricoxib somit keinen schmerzlindernden Effekt im Vergleich zu Placebo zu haben.

Die Ergebnisse bestätigen die Erkenntnisse von Seidel et al. Auch sie beschrieben in ihrer Studie, dass eine positive Wirksamkeit von Ketoprofen auf Muskelschmerz lediglich in der Frühphase nach exzentrischer Belastung zu sehen war. Innerhalb der ersten 24 Stunden nach exzentrischer Belastung beobachteten sie in der Behandlungsgruppe mit Ketoprofen einen Trend zur Reduktion des 
Muskelschmerzes. Dagegen wiesen sie - bei Betrachtung des kompletten Nachbeobachtungszeitraums von 144 Stunden nach exzentrischer Belastung einen statistisch signifikant erhöhten Muskelschmerz $(p=0,024)$ unter Behandlung mit Ketoprofen im Vergleich zu Placebo nach. Somit zeigte sich, wie auch in dieser Studie, in der Frühphase nach exzentrischer Belastung ein schmerzlindernder Effekt durch die Behandlung mit NSAR. Interessanterweise beobachteten Seidel et al. in ihrer Studie insgesamt jedoch nicht nur einen ausbleibenden, sondern insbesondere in der späteren Beobachtungsphase sogar einen negativen Behandlungseffekt von NSAR bei Muskelschmerz nach exzentrischer Belastung (Abb. 16). Diese Erkenntnis bekräftigt Vermutungen, dass NSAR eine Abnahme des Muskelschmerzes in der Erholungsphase nach exzentrischer Belastung sogar verzögern könnten (Seidel et al. 2012).

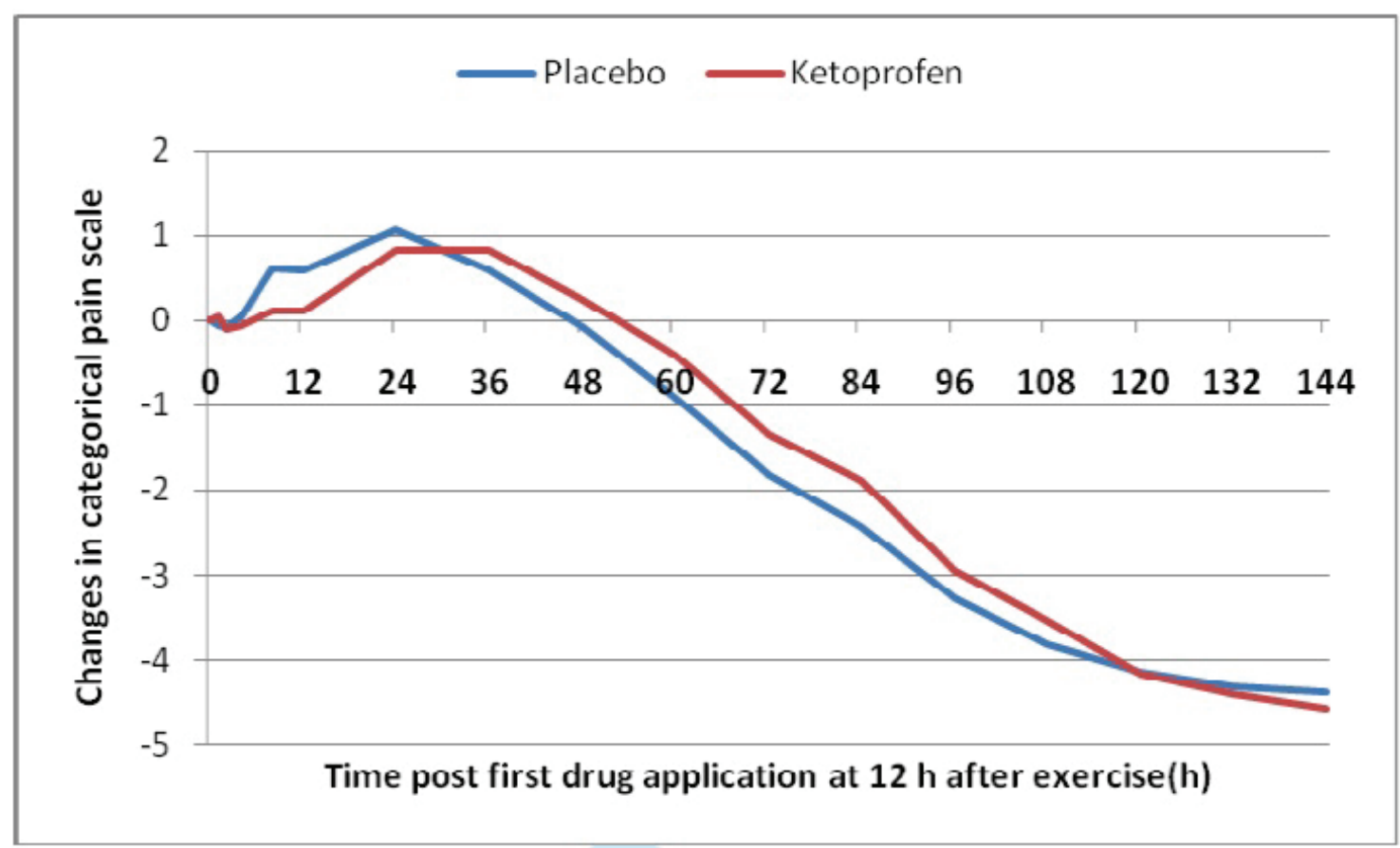

Abbildung 16: Zeitlicher Verlauf - Veränderung von Muskelschmerz unter Behandlung mit Ketoprofen vs. Placebo (aus Seidel et al. 2012)

Hinweise darauf, dass die Erholungsphase von DOMS durch die Einnahme von NSAR tatsächlich verzögert ablaufen könnte, liefern auch Ergebnisse dieser Studie. Bei der Betrachtung von Veränderungen der Hitzeschmerzschwelle am 
max. Druckpunkt des Muskels nach exzentrischer Belastung konnte kein signifikanter Unterschied zwischen den beiden Behandlungsgruppen ( $p=0,4586$ ) über den gesamten Zeitraum nachgewiesen werden. Bezieht man auch hier den zeitlichen Verlauf (Früh-/Spätphase) in die Betrachtung mit ein, so zeigte sich ab 8 Stunden nach erster Medikamentenapplikation eine leicht niedrigere Schmerzschwelle unter Etoricoxib-Einnahme im Vergleich zu Placebo. Dieser statistisch nicht signifikante Unterschied blieb bis zum Ende der Nachbeobachtungsphase nach 168 Stunden auf einem leichten bis moderaten Niveau bestehen (siehe Abb. 8). Diese Ergebnisse sprechen für einen Trend zur verlangsamten Wiederherstellung des ursprünglichen Schmerzschwellenniveaus unter Einnahme von Etoricoxib. Etoricoxib scheint einen Wiederanstieg der Schmerzschwelle in der Erholungsphase nach exzentrischer Belastung hinauszuzögern.

Obwohl kein statistisch signifikanter Effekt von Etoricoxib auf Muskelschmerz nach exzentrischer Belastung gezeigt wurde, kann die Arbeitshypothese $\mathrm{H}_{1}$, unter Berücksichtigung der o. g. Trends, hinsichtlich des Muskelschmerzes nur bedingt angenommmen werden.

Ähnliche Ergebnisse wie die für Muskelschmerz konnten in der Studie hinsichtlich der Erholung der Maximalkraft nach exzentrischer Belastung beobachtet werden. Bei der Betrachtung der Maximalkraft über den gesamten Beobachtungseitraum konnte kein Nachweis für einen signifikanten Unterschied zwischen den beiden Behandlungsgruppen $(p=0,6575)$ erbracht werden. Unter Einbeziehung des zeitlichen Verlaufes in die Ergebnisbetrachtung konnte jedoch auch hier ein Trend zur verzögerten Erholung und verlangsamten Wiederherstellung der maximalen Muskelkraft unter Etoricoxib-Einnahme nach exzentrischer Belastung gezeigt werden. Die Werte der maximalen Muskelkraft waren ab 24 Stunden nach Behandlungsbeginn bis zum Ende der Nachbeobachtungsphase unter Etoricoxib-Einnahme im Vergleich zu Placebo leicht erniedrigt. Zudem wurden die Ausgangswerte der Maximalkraft unter Behandlung mit Etoricoxib - im Gegensatz zu Placebo - auch zum Ende der Nachbeobachtungsphase nicht wieder erreicht (siehe Abb. 9). Die Einnahme von Etoricoxib 
scheint die Regeneration der Muskelkraft nach exzentrischer Belastung zu verlangsamen und ein Erreichen der ursprünglichen Muskelkraft über einen Zeitraum von über 8 Tagen hinauszuzögern. Aus diesem Grund kann die Arbeitshypothese $\mathrm{H}_{1}$ hinsichtlich der Erholung der Muskelkraft nur bedingt angenommen werden.

Ein statistisch signifikanter antientzündlicher Effekt von Etoricoxib im Vergleich zu Placebo konnte anhand der hs CrP-Reduktion ( $p=0,0203)$ nachgewiesen werden (siehe Abb. 11). Außerdem zeigte sich der Trend eines verminderten Leukozytenanstieg unter Etoricoxib-Einnahme im Vergleich zur Placebo $(p=$ 0,0981). Die während DOMS ablaufende Entzündungsreaktion wird durch die Einnahme von Etoricoxib nach exzentrischer Belastung gehemmt und im Vergleich zu Placebo abgeschwächt. Aus diesem Grund muss die Arbeitshypothese $\mathrm{H}_{1}$ hinsichtlich der Entzündungsreaktion abgelehnt werden.

Die in der Studie gezeigten Trends zu einer verzögerten Wiederherstellung der Schmerzschwelle und zu einer verlangsamten Erholung der maximalen Muskelkraft unter Einnahme von Etoricoxib lassen vermuten, dass die gezeigte Unterdrückung der Entzündungsreaktion durch Etoricoxib zu einer verzögerten Regeneration des Muskelschadens nach exzentrischer Belastung führen könnte. Der Nachweis, dass die ablaufende Entzündungsreaktion nach exentrischer Belastung in der Entstehung des verzögerten Muskelschmerzes bei DOMS eine wichtige Rolle spielt, wurde mehrfach erbracht (Smith 1991). Dass die Entzündungsreaktion eine ebenso essentielle Bedeutung im Rahmen des Regenerationsprozesses im Muskel nach exzentrischer Belastung besitzt (Howatson und van Someren 2008), ist bei Betrachtung der Ergebnisse ein sehr interessanter Aspekt. Trappe et al. beobachteten, dass durch den antientzündlichen Effekt von Ibuprofen die Produktion von $\mathrm{PGF}_{2 \mathrm{a}}$ und $\mathrm{PGE}_{2}$ im Muskel nach exzentrischer Belastung gehemmt wird (Trappe et al. 2001). Diese Reduktion von PGE 2 führt zu einer verminderten Aktivierung von Satellitenzellen, Muskelstammzellen und Wachstumsfaktoren. All diese sind Zellen, die eine Schlüsselrolle in der Regeneration der verletzten Muskelfaser nach exzentrischer Belastung spielen 
und die, bedingt durch eine Einnahme von NSAR, in kleinerer Zahl zur Verfügung stehen (Prisk und Huard 2003). Infolgedessen werden die Proteinsynthese im Muskel (Trappe et al. 2001) und die Hypertrophie des Muskels, die normalerweise nach exentrischer Belastung beobachtet werden, durch die Einnahme von NSAR unterdrückt (Soltow et al. 2006). Die Vermutung, dass NSAR in den Muskelstoffwechsel und den Regenerationsprozess eingreifen, wird durch eine Studie von Nieman et al. unterstützt. Sie konnten nachweisen, dass bei Ultramarathonläufern, die NSAR vor und während eines 160 kmRennens einnahmen, im Vergleich zu Placebo nach dem Lauf kein Unterschied im Ausmaß des Muskelschadens oder des Muskelschmerzes zu sehen war. Die Plasma-Level für fünf von sieben Zytokinen waren unter NSAR-Einnahme jedoch erhöht (Nieman et al. 2006). Diese Studien geben Hinweise darauf, auf welche Weise sich der entzündungshemmende Effekt der NSAR auf die Reparations- und Regenerationsprozesse im Muskel nach exzentrischer Belastung auswirkt.

Die Ergebnisse dieser Studie bekräftigen bisherige Annahmen aus Tiermodellen, in denen die Wirksamkeit von NSAR nach exzentrischer Belastung als „Zweischneidiges Schwert“ beschrieben wurde (Mishra et al. 1995). In der Frühphase nach exzentrischer Belastung scheint ein überwiegend positiver Effekt der NSAR-Einnahme im Vordergrund zu stehen. Innerhalb der ersten 24 Stunden nach exzentrischer Belastung wird der Muskelschmerz durch die Einnahme von NSAR vermeintlich abgeschwächt. Dieser schmerzlindernde Effekt scheint jedoch auf Kosten der Regeneration des Muskels zu geschehen (Hertel 1997). Die gezeigten Trends zu einer verzögerten Abnahme des Muskelschmerzes und einer verlangsamten Wiederherstellung der Muskelkraft durch die Einnahme von NSAR nach exzentrischer Belastung wurden auch in einer Publikation von Mackey et al. beschrieben (Mackey et al. 2012). Diese, insbesondere in der späteren Phase der Erholung von DOMS zu beobachtende, vermeintlich negativen Effekte, scheinen gegenüber dem positiven Effekt von NSAR in der Frühphase nach exzentrischer Belastung zu überwiegen. 
Die Einnahme von NSAR würde somit eine kurzzeitige Linderung des Muskelschmerzes in der Anfangsphase von DOMS bewirken, eine schnellere Wiederherstellung der Muskelfunktion und ein schnelleres Erreichen von Beschwerdefreiheit ist jedoch nicht zu erwarten. Im Gegenteil, die Symptome von DOMS scheinen durch die Einnahme von NSAR sogar über einen längeren Zeitraum zu bestehen. Auf Grundlage dieser Erkenntnisse scheint die Indikation zur Anwendung von NSAR bei DOMS nicht gegeben zu sein.

\section{3 $\quad \mathrm{H}_{2}$ : Einfluss der Studienmethodik auf Ergebnisse bisheriger Studien}

Im folgenden Abschnitt soll die Arbeitshypothese $\mathrm{H}_{2}$ diskutiert werden: Die unterschiedlich starke antientzündliche Potenz der NSAR, der Zeitpunkt der ersten NSAR-Einnahme nach exzentrischer Belastung und die Häufigkeit der NSARGabe haben keinen Einfluss auf den zeitlichen Verlauf von DOMS.

In der Literatur herrscht bisher wenig Einigkeit darüber, ob eine Wirksamkeit von NSAR bei Muskelschmerz nach exzentrischer Belastung besteht. Die Ergebnisse vieler Studien stehen häufig im Widerspruch zueinander. In rund der Hälfte der durchgeführten Studien wurden keine Hinweise auf eine Wirksamkeit von NSAR in der Behandlung von DOMS gefunden. In der anderen Hälfte der Studien konnte eine statistisch signifikante Reduktion von DOMS unter Einnahme von NSAR nachgewiesen werden. Wahrscheinlich ist, dass Unterschiede in der Methodik der Studien ursächlich für die voneinander abweichenden Ergebnisse sind. Die Studien unterscheiden sich in der Methodik in folgenden Gesichtspunkten: Es wurden verschiedene exzentrische Belastungsmodelle zur Erzeugung von DOMS verwendet und es wurden unterschiedliche NSAR untersucht. Die Medikamente unterschieden sich hinsichtlich ihres Wirkstoffes. Es wurden unterschiedliche Applikationsformen gewählt. Der Einnahmezeitpunkt und die Häufigkeit der Applikation variierten teilweise erheblich. Letztendlich liegen auch deutliche Unterschiede vor, zu welchem Zeitpunkt oder über welchen 
Zeitraum die Effektbestimmung erfolgte und wie lange ein möglicher Behandlungseffekt nachbeobachtet wurde (Mackey et al. 2012).

Tab. 27 stellt die verschiedenen Studien einander gegenüber und vergleicht sie hinsichtlich der o. g. Variablen in der Studienmethodik. Um die Angaben über die Wirksamkeit der NSAR auf Muskelschmerz und Muskelkraft zwischen den Studien vergleichbar darzustellen, wurde die Effektstärke der Mittelwertunterschiede („Cohens d“) für die Studien bestimmt (Lanier 2003). Nach Cohen beschreibt $d=0,2$ einen kleinen Effekt, $d=0,5$ einen mittleren Effekt und $d=0,8$ einen starken Effekt (Cohen 1988). Falls die Effektstärke aus den dargestellten Ergebnissen der Publikation nicht berechenbar war, wurde - soweit möglich der $p$-Wert eingefügt. 


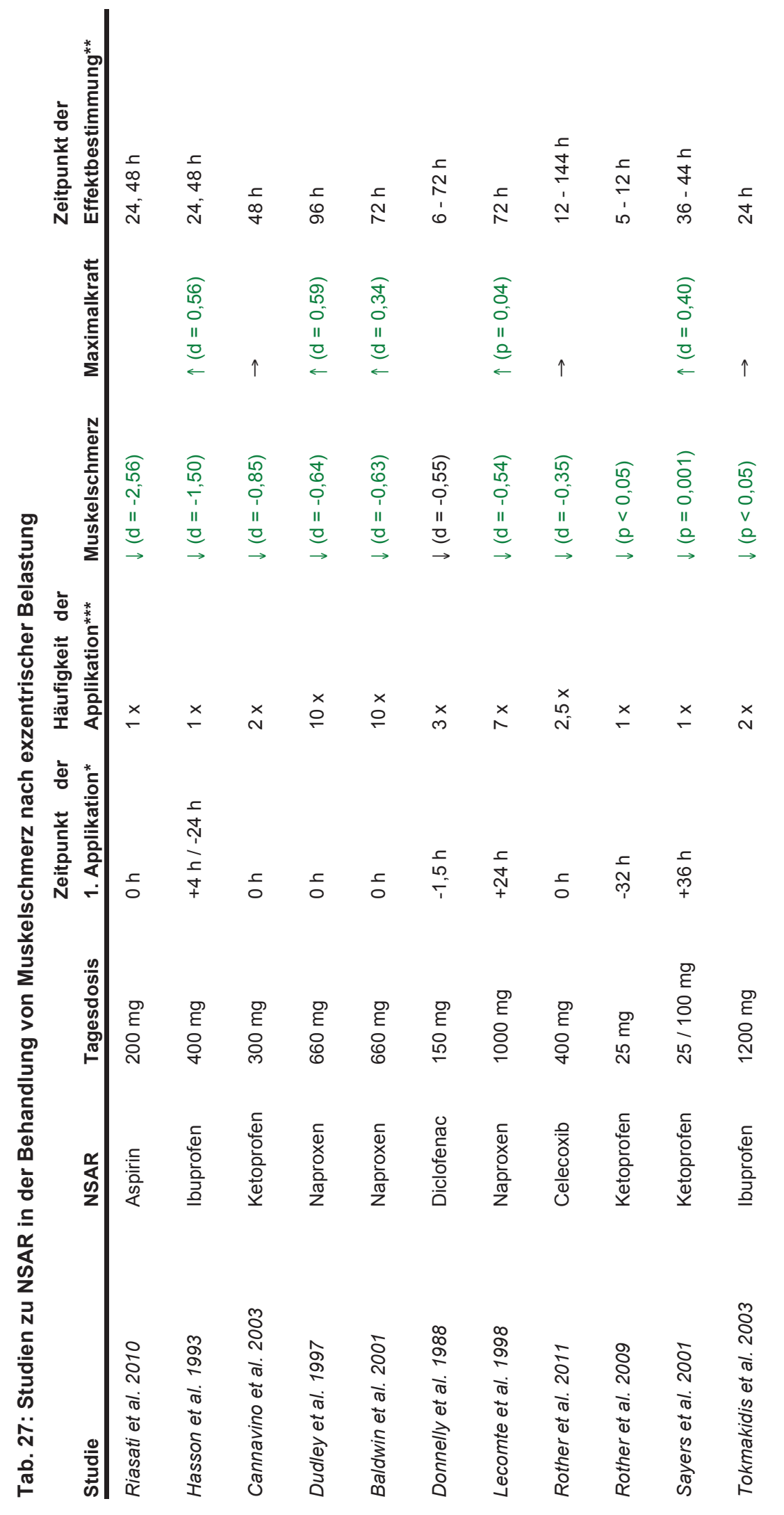




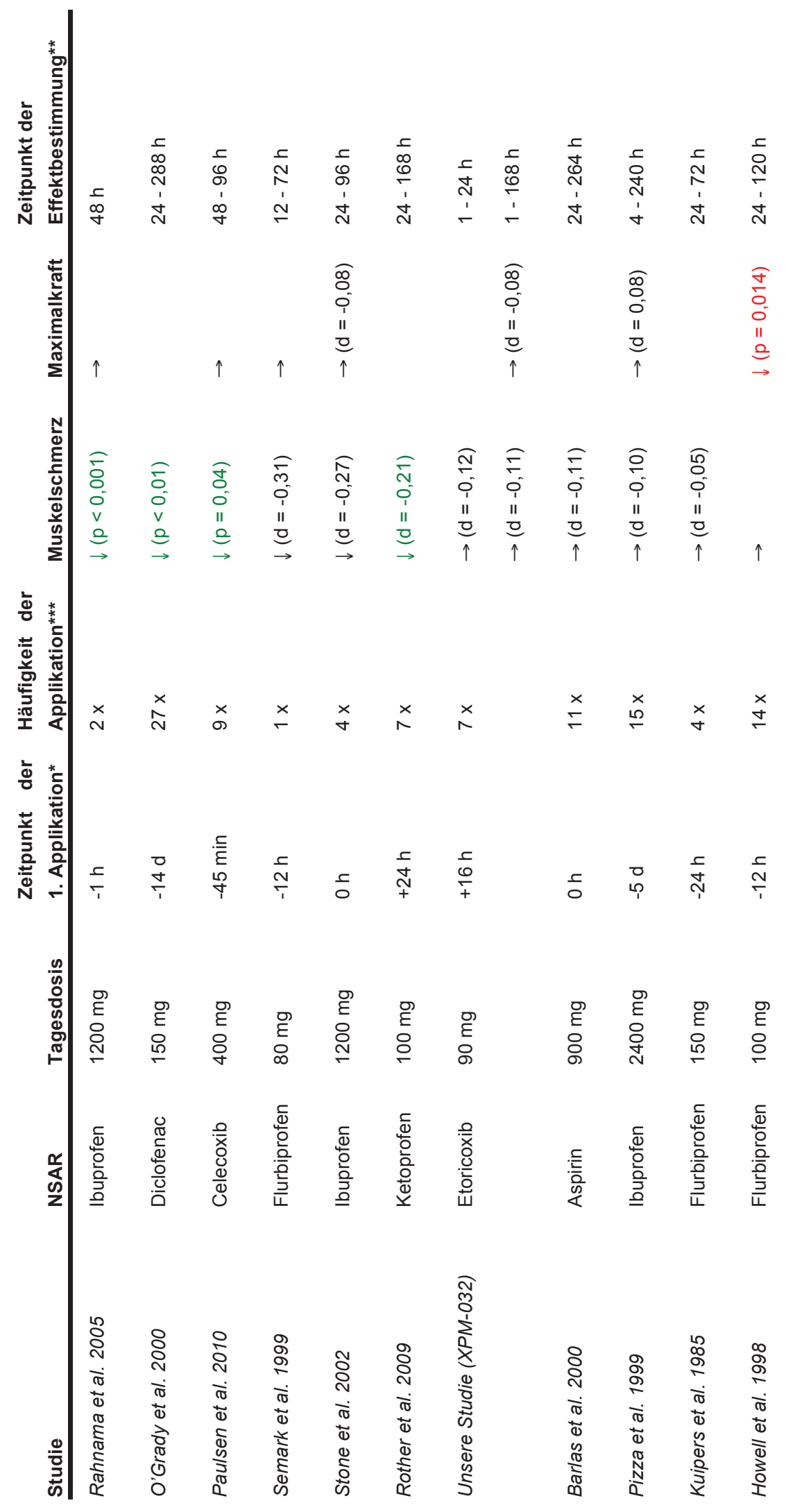




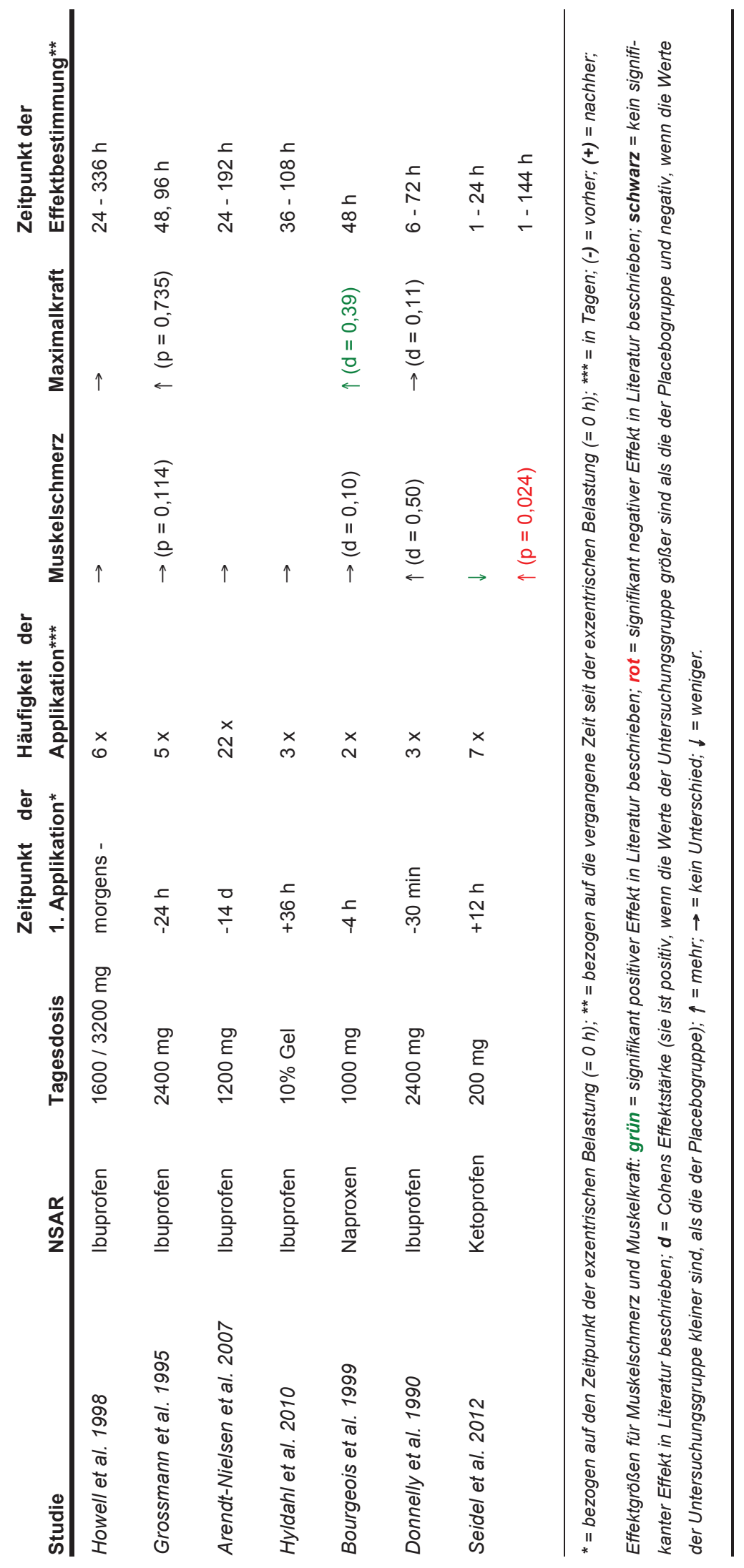


Diese Studie wie auch die Studie von Seidel et al. zeigten insbesondere in der Frühphase nach exzentrischer Belastung einen Trend zur Schmerzreduktion unter der Behandlung mit NSAR. In der späteren Regenerationsphase konnte dieser schmerzlindernde Effekt von NSAR jedoch nicht mehr beobachtet werden (Seidel et al. 2012). Diese Erkenntnis legt nahe, dass der Zeitpunkt der Effektbestimmung ausschlaggebend für die Beobachtung eines postiven oder eines ausbleibenden Effekts sein kann. Tab. 27 zeigt, dass in den Studien, die einen positiven Effekt unter NSAR-Einnahme beschreiben, die Effektbestimmung überwiegend relativ früh nach Behandlungsbeginn vorgenommen wurde. Unter Berücksichtigung des o. g. zeitlichen Verlaufs könnten bei einer Beschränkung der Effektbestimmung auf die Frühphase der Behandlung die vermutlich lediglich in dieser Frühphase auftretenden positiven Behandlungseffekte von NSAR erfasst und entsprechend einseitig bewertet worden sein.

Auf der anderen Seite wurde in den mehrheitlichen Studien, die keine oder negative Behandlungseffekte von NSAR beschreiben, die Effektbestimmung tendenziell später durchgeführt oder ein Gesamteffekt über einen längeren Zeitraum von mehreren Tagen bestimmt. Auch diese Erkenntnis lässt die Vermutung zu, dass Studien, die den Effekt von NSAR lediglich in der Frühphase der Behandlung beobachteten, einen möglichen ausbleibenden oder negativen Behandlungseffekt über einen längeren Zeitraum von einigen Tagen übersehen und vernachlässigt haben könnten. Eine Beobachtung des zeitlichen Verlaufs sowohl in der frühen Phase als auch über einen Zeitraum von einigen Tagen nach exzentrischer Belastung scheint somit erforderlich, um die vermutlich ambivalenten Effekte von NSAR auf DOMS in Studien nachzuweisen.

In dieser Studie wurde die Studienmedikation insgesamt sieben mal appliziert. Betrachtet man die Häufigkeit der Medikamentenapplikation in anderen Studien, so zeichnen sich auch hier deutliche Unterschiede ab. Tab. 27 zeigt, dass in den überwiegenden Studien, in denen ein positiver Effekt unter NSAR-Einnahme beschrieben wird, die Medikamentenapplikation tendenziell seltener erfolgte. In den 
mehrheitlichen Studien, die keine oder negative Behandlungseffekte von NSAR beschreiben, wurde die Studienmedikation tendenziell häufiger verabreicht. Dieser angedeutete Trend könnte vermuten lassen, dass bei einer kurzzeitigen Einnahme von NSAR (ein- bis zweimal) nach exzentrischer Belastung eher die positiven Behandlungseffekte im Vordergrund stehen könnten. Bei einer längerfristigen Einnahme von NSAR dagegen könnten eher die negativen Effekte überwiegen. Ob dieser Trend nicht auch eher auf die Nachbeobachtungszeit und weniger auf die Häufigkeit der Medikamentenapplikation zurückzuführen ist, kann an dieser Stelle nicht geklärt werden.

Ein wesentlicher Einfluss des Zeitpunktes der ersten Medikamentenapplikation auf den Behandlungseffekt konnte unter Berücksichtigung von Tab. 27 im Vergleich der einzelnen Studien nicht festgestellt werden. Bei den meisten Studien erfolgte die erste Medikamentenapplikation kurz vor oder kurz nach der exzentrischen Belastung. Ob es einen wesentlichen Unterschied gibt, wann genau diese erste Medikamentengabe durchgeführt wurde, konnte anhand der diesbezüglichen Auswertung der Studien nicht geklärt werden.

Diese Studie zeigte, dass die Regeneration der maximalen Muskelkraft durch die Einnahme von NSAR verzögert werden könnte. Eine Studie von Rother et al. führte neben der Beobachtung des Schmerzverlaufs auch eine Verlaufsbeobachtung der Muskelkraft durch, die mit der dieser Studie vergleichbar ist (Rother et al. 2011). Rother et al. beobachteten hinsichtlich des Muskelschmerzes unter der Behandlung mit Celecoxib einen in Verlauf und Ausmaß im Vergleich zu dieser Studie ähnlichen Effekt. Der in dieser Studie für Etoricoxib gezeigte Trend einer verzögerten Erholung der Muskelkraft konnte von Rother et al. für Celecoxib jedoch nicht nachgewiesen werden. Das Ausbleiben dieses Trends könnte durch die unterschiedlich starke, antientzündliche Aktivität der untersuchten NSAR begründet sein. In ex-vivo Tests wurde anhand der Hemmstärke auf die $\mathrm{PGE}_{2}$-Synthese die antientzündliche Potenz einiger NSAR bei der jeweiligen Standarddosis bestimmt (van Hecken et al. 2000, Schwartz et al. 2008). Tab. 28 stellt die entsprechende 
Rangfolge der NSAR dar, absteigend geordnet nach ihrer antientzündlichen Aktivität.

Tab. 28: Vergleich der antientzündlichen Potenz von NSAR; Rang 1 = stärkste antientzündliche Aktivität (nach van Hecken et al. 2000 und Schwartz et al. 2008)

\begin{tabular}{ll} 
Rang & NSAR \\
\hline 1 & Diclofenac \\
2 & Etoricoxib \\
3 & Celecoxib \\
4 & Naproxen = Ibuprofen
\end{tabular}

Die scheinbar höhere antientzündliche Potenz von Etoricoxib im Vergleich zu Celecoxib könnte erklären, warum eine verlangsamte Wiederherstellung der maximalen Muskelkraft in der Studie von Rother et al. nicht beobachetet werden konnte. Durch die vermutlich schwächere Hemmung der Entzündungsreaktion durch Celecoxib könnte der Regenerationsprozess im Muskel nach der exzentrischen Belastung weniger stark beeinträchtigt worden sein. Dadurch könnte eine verlangsamte Wiederherstellung der maximalen Muskelkraft in der Studie von Rother et al. nicht aufgetreten sein.

Ein Vergleich der übrigen Studien, wie in Tab. 27 dargestellt, deutet darauf hin, dass bei der Verwendung von NSAR mit bestimmten Wirkstoffen relativ mehr positive Behandlungseffekte beobachtet wurden, als bei NSAR mit bestimmten anderen Wirkstoffen. Positive Effekte wurden vorwiegend in Studien mit Ketoprofen, Naproxen, Diclofenac und Celecoxib beschrieben. In der Mehrzahl der Studien zu Flurbiprofen und Ibuprofen wurden hingegen keine Behandlungseffekte beobachtet. Es liegen zwar für die meisten Wirkstoffe keine Studien zur antientzündlichen Aktivität bei Standarddosis vor, jedoch könnte auch diese Beobachtung Grund zu der Annahme geben, dass die antientzündliche Aktivität der NSAR eine Rolle in der Behandlungswirksamkeit spielt. Stärker potente NSAR könnten einen ausgeprägteren Behandlungseffekt nach exzentrischer Belastung zeigen, als NSAR mit niedrigerer antientzündlicher Aktivität. 
Zusammenfassend scheinen die unterschiedlichen Variablen in der Studienmethodik Einfluss auf die Feststellung und die Bewertung von Behandlungseffekten von NSAR bei Muskelschmerz nach exzentrischer Belastung zu haben. Zu vermuten ist, dass bei Studien mit einer kürzeren Nachbeobachtungszeit, einer weniger häufigen Medikamentenapplikation und der Gabe eines weniger antientzündlich wirksamen NSAR, ein eher positiver Behandlungseffekt bei DOMS beobachtet werden kann. Aus diesem Grund sollte die Arbeitshypothese $\mathrm{H}_{2}$ abgelehnt werden.

\section{4 $\quad \mathrm{H}_{3}$ : Alter und Muskelschmerz nach exzentrischer Belastung}

Im folgenden Abschnitt soll die Arbeitshypothese $\mathrm{H}_{3}$ diskutiert werden: Es gibt hinsichtlich des Alters keinen Unterschied zwischen der Wirksamkeit von Etoricoxib und Placebo auf Muskelschmerz, auf die Erholung der Muskelkraft und auf Entzündungsmarker im Blut nach exzentrischer Belastung.

$\mathrm{Da}$ in dieser Studie für die Gesamtpopulation keine statistisch signifikanten, behandlungsbezogenen Unterschiede beobachtet werden konnten, trifft diese Feststellung folglich auch für die Gruppe der jüngeren und der älteren Erwachsenen zu. Die Arbeitshypothese $\mathrm{H}_{3}$ sollte somit, unter Berücksichtigung der oben geführten Diskussion, wie die Arbeitshypothese $\mathrm{H}_{1}$ bewertet werden.

Eine geplante Analyse der Wirksamkeit von Etoricoxib im Vergleich zu Placebo in den Subgruppen „Jüngere“ und „Ältere“ wurde aus diesem Grund nicht durchgeführt. Gleichwohl waren bei der Betrachtung der Subgruppen Jüngere und Ältere innerhalb der Placebo-Gruppe nicht behandlungsbezogene, sondern altersbezogene, physiologisch relevante Unterschiede auffällig. Die Beobachtungen lassen vermuten, dass ältere Erwachsene anders auf exzentrische Belastung reagieren als jüngere Erwachsene. Jüngere Menschen scheinen nach exzentrischer Belastung eine höhere Schmerzempfindlichkeit im Muskel vorzuweisen als ältere Menschen (siehe Abb. 12). Nach exzentrischer Belastung (bewertet direkt vor der ersten Medikamenteneinnahme) gaben die Jüngeren höhere Werte für Bewegungsschmerz $(7,0 \pm 1,49)$ und Ruheschmerz $(2,9 \pm 2,01)$ an als die Älteren 
$(6,3 \pm 1,52$, bzw. $2,0 \pm 2,58)$. Das Schmerzerleben scheint somit während der Frühphase von DOMS bei den Jüngeren ausgeprägter zu sein als bei den Älteren.

Diese Erkenntnis geht einher mit der Beobachtung einer statistisch signifikant stärker reduzierten Schmerzschwelle am max. Druckpunkt ( $p=0,0147$ ) in der Gruppe der Jüngeren $(48,45 \pm 1,91)$ im Vergleich zu der Gruppe der Älteren $(49,73 \pm 1,38)$ nach exzentrischer Belastung (vor Beginn der Behandlung) (siehe Abb. 12). Die subjektive Bewertung der Jüngeren, die ihre Muskelschmerzen nach exzentrischer Belastung als stärker ausgeprägt einschätzten als die Älteren, wird somit auch objektiv gestützt. In der frühen Phase nach Erzeugung von DOMS wurden auf den Muskel applizierte Hitzereize von den Jüngeren schon bei Temperaturen, die von den Älteren noch nicht als schmerzhaft empfunden wurden, nicht mehr toleriert. Die Hitzeschmerzschwelle am Punkt der maximalen Ausprägung des Muskelkaters ist somit durch die exzentrische Belastung bei Jüngeren stärker erniedrigt als bei den Älteren.

Diese Erkentnisse decken sich mit denen von Hyldahl et al., die in ihrer Studie ein geringeres Erleben von Muskelschmerz nach exzentrischer Belastung der Älteren im Vergleich zu den Jüngeren beobachteten. Sie konnten einen statistisch signifikant niedrigeren Muskelschmerz $(p<0,01)$ der älteren Kohorte im Vergleich zur jüngeren Kohorte nachweisen (Hyldahl et al. 2010). Mehrere Erklärungsansätze für das verminderte Schmerzerleben der Älteren im Rahmen von DOMS sind in der Literatur zu finden. Lavender und Nosaka, die in ihrer Studie ebenfalls ein statistisch signifikant niedrigeres Schmerzniveau der Älteren nach exzentrischer Belastung beschrieben $(p<0,05)$, versuchten sich diesen Zustand anhand von Aussagen mehrerer ihrer älteren Probanden zu erklären. Diese sagten nach der Studie aus, „ihre Muskeln sind steif, aber verglichen mit anderen im Leben erlittenen Schmerzen ist das Schmerzniveau nicht hoch“ (Lavender und Nosaka 2008). Diese Aussage lässt den Schluss zu, dass ältere Menschen möglicherweise aufgrund ihrer bisherigen Erfahrungen im Leben Schmerzen eher als „tolerabel“ einschätzen könnten als jüngere Menschen. Harkins and Price postulierten: „age is not an analgesic“. Sie erklärten, dass es keine Unterschiede in der Schmerzemp- 
findung zwischen Älteren und Jüngeren gebe (Harkins und Price 1992). Vermutbar ist somit, dass die älteren Erwachsenen mehr Schmerzen aushalten und damit lediglich eine erhöhte Schmerztoleranz besitzen, im Grunde jedoch kein Unterschied in der Schmerzempfindung zwischen Jüngeren und Älteren besteht.

Auf der anderen Seite erklärten Gibson und Helme, dass die Art des Schmerzerlebens sich im Alter zu ändern scheint. Die Häufigkeit und die Intensität von Schmerzsymptomen im Zusammenhang mit Myokardinfarkten, abdominellen Infektionen, muskuloskeletalen Beschwerden oder postoperativen Komplikationen ändert sich mit dem Alter (Gibson und Helme 2001). Mitverantwortlich für diese veränderte Art des Schmerzerlebens scheint eine altersbedingt veränderte Schmerzwahrnehmung zu sein. Eine abgeschwächte Schmerzsensorik, eine verlangsamte Schmerzweiterleitung oder eine verminderte zentrale Schmerzverarbeitung scheinen im Rahmen von physiologischen Entwicklungen im Alter hierbei eine Rolle zu spielen. Der Sinn dieser Veränderungen könnte eine Anpassung auf vermehrte Schmerzzustände im Alter sein (Basler et al. 1999). Sicherlich greifen auch pathologische Veränderungen im Rahmen von chronischen Erkrankungen, wie z. B. diabetische Neuropathien, häufig in Vorgänge der Nozizeption ein und beeinflussen diese.

Zusammengenommen sind die rund um das Schmerzerleben ablaufenden Prozesse sehr komplex. Ob die Ergebnisse der Studie, die zeigen, dass ältere Erwachsene nach exzentrischer Belastung subjektiv weniger Schmerz angaben und eine weniger stark erniedrigte Schmerzschwelle vorwiesen, auf eine verminderte Schmerzwahrnehmung im Alter, eine erhöhte Schmerztoleranz im Alter oder auf eine Kombination von beidem zurückzuführen ist, kann hier nicht abschließend geklärt werden.

Desweiteren zeigt die Studie, dass sich die jüngeren Erwachsenen im Vergleich zu den älteren Erwachsenen nach exzentrischer Belastung schneller erholen. Der Muskelschmerz in Ruhe nahm bei den Jüngeren im zeitlichen Verlauf von DOMS schneller wieder ab, als bei den Älteren (siehe Abb. 13). Diese schnellere Reduk- 
tion des Muskelschmerzes war statistisch signifikant (Summe: $p=0,016$; Tag 3-8: $p=0,008$ ). Jüngere Erwachsene scheinen somit eine bessere Erholungsfähigkeit der nach exzentrischer Belastung geschädigten Muskulatur zu besitzen als ältere Erwachsene. Sie zeigten eine beschleunigte Abnahme der Muskelschmerzen in der Regenerationsphase von DOMS.

Ein ähnlicher Trend konnte im zeitlichen Verlauf für die Wiederherstellung der maximalen Muskelkraft nach exzentrischer Belastung beobachtet werden. Im Vergleich zu den älteren Menschen war bei den jüngeren Menschen der Trend zu einer schnelleren Wiederherstellung der maximalen Muskelkraft erkennbar (Summe: $p=0,1303$; Tage 3-8: $p=0.0584$ ) (siehe Abb. 14). Die hinsichtlich des Ruheschmerzes gezeigte verbesserte Erholungsfähigkeit des ,jüngeren“ Muskels nach exzentrischer Belastung scheint sich auch in der Wiederherstellung der maximalen Muskelkraft widerzuspiegeln. In der Regenerationsphase von DOMS scheint sich die maximale Muskelkraft der jüngeren Erwachsenen schneller wiederherzustellen als die der älteren Erwachsenen.

Die Ergebnisse der Studie bekräftigen die Erkenntnisse von Dedrick und Clarkson. Sie konnten in ihrer Studie bei älteren Probanden im Vergleich zu den jüngeren Probanden ebenfalls eine langsamere Erholung der maximalen Muskelkraft nach exzentrischer Belastung feststellen (Dedrick und Clarkson 1990). Sie erklären sich dies mit einer allgemein abnehmenden Fähigkeit des Körpers, Gewebe zu regenerieren. Hinsichtlich der physiologischen Alterungsprozesse, die im Muskel zu beobachten sind, steht eine Abnahme der Muskelmasse im Vordergrund. Die vorhandenen restlichen Muskelfasern sind häufig atrophiert. Letztendlich nimmt die Muskelkraft im Rahmen dieser altersspezifischen Veränderungen der Muskulatur deutlich ab (Lexell et al. 1988). Ploutz-Snyder et al. sehen darin den Grund für ein erhöhtes Verletzungsrisiko älterer Menschen. Sie postulieren in ihrer Studie, dass ältere Menschen deutlich anfälliger für Muskelverletzungen seien (Ploutz-Snyder et al. 2001). Manfredi et al. fügen an, dass durch die altersspezifischen Veränderungen im Muskel größere strukturelle Muskelschäden nach exzentrischer Belastung entstehen (Manfredi et al. 1991). Wenn also bei älteren Erwachsenen nach 
exzentrischer Belastung ein strukturell größerer muskulärer Schaden auftritt, scheint es wahrscheinlich, dass die Reparatur eines solchen Schadens einen längeren Zeitraum beansprucht als ein vermeintlich geringerer muskulärer Schaden bei jüngeren Erwachsenen. Bei einer zusätzlich verminderten Regenerationsfähigkeit des Körpergewebes im Alter wird der Erholungsprozess im Muskel womöglich noch weiter verlangsamt. Somit erscheint es nachvollziehbar, dass der Regenerationsprozess des „älteren“ Muskels nach exzentrischer Belastung langsamer abläuft als bei jüngeren Erwachsenen.

\section{5 $\quad \mathrm{H}_{4}$ : NSAR bei Muskelschmerz nach exzentrischer Belastung}

Im folgenden Abschnitt soll die Arbeitshypothese $\mathrm{H}_{4}$ diskutiert werden: NSAR sollten bei Muskelschmerz nach exzentrischer Belastung nicht angewandt werden.

Trotz der vermeintlich schmerzlindernden Wirkung von NSAR in der Frühphase nach exzentrischer Belastung sollte aufgrund der scheinbar überwiegend negativen, die Regeneration des geschädigten Muskels hemmenden Effekte von NSAR auf die Einnahme antientzündlicher Schmerzmedikamente im Rahmen von DOMS verzichtet werden. Außerdem sollte bei einem ohne Folgeschädigung auftretenden, selbstlimitierend ablaufenden, zeitlich begrenzten Prozess wie DOMS - auch aufgrund des weiten Nebenwirkungsspektrums der NSAR - die Anwendung einer solchen Medikation kritisch betrachtet werden. Stattdessen sollte während des Auftretens von DOMS auf eine außergewöhnliche (sportliche) Belastung verzichtet werden, um Verletzungen zu vermeiden.

Unter Berücksichtigung der Untersuchungsergebnisse sowie o. g. Faktoren kann eine Einnahme von NSAR zur Behandlung von Muskelschmerz nach exzentrischer Belastung nicht empfohlen werden. Die Arbeitshypothese $\mathrm{H}_{4}$ sollte von daher angenommen werden. 


\section{SCHLUSSFOLGERUNGEN}

Diese Arbeit untersuchte die Wirksamkeit antientzündlicher Therapie bei Muskelschmerz nach exzentrischer Belastung.

Das Studienmodell ermöglichte eine nahezu vollständige Standardisierung der exzentrischen Belastung und erwies sich dadurch als geeignet, bei den Probanden DOMS in ähnlich starker Ausprägung zu erzeugen. Das Auftreten eines RBEs wurde durch die Belastung von jeweils nur einem Bein vermieden. Somit war die Durchführung der Studie im crossover-Design möglich.

Mängel offenbarte das Modell jedoch bei der Methode zur Bestimmung des Bewegungsschmerzes. In zukünftigen Studien sollte die Bestimmung von Bewegungsschmerz unabhängig und nicht zeitgleich mit der Bestimmung der Maximalkraft erfolgen. Sie könnte z. B. während der Aufwärmphase bestimmt werden.

Die Bestimmung der Hitzeschmerzschwelle erwies sich als eine geeignete Methode, um das Ausmaß der erzeugten Muskelschmerzen objektiv darzustellen. Ob andere Methoden zur Schmerzschwellenbestimmung (z. B. mechanische) sensitiver sein könnten, muss in weiterführenden Vergleichsstudien gezeigt werden.

Der Laborparameter hs CrP erwies sich als der geeignetste unter den gemessenen Entzündungsmarkern, um die nach exzentrischer Belastung ablaufende Entzündungsreaktion darzustellen.

Die Studie zeigte bezüglich des Muskelschmerzes nach exzentrischer Belastung im Vergleich zu Placebo keine statistisch signifikanten Behandlungseffekte von Etoricoxib. Etoricoxib konnte die ablaufende Entzündungsreaktion im Vergleich zu Placebo signifikant abschwächen. In der frühen Phase der Behandlung (erste 24 Stunden) scheint der Muskelschmerz dadurch geringfügig reduziert zu sein. Wenn überhaupt, so scheint die Regeneration der Muskelkraft und die Wiederherstellung der ursprünglichen Schmerzschwelle in der Erholungsphase nach exzentrischer Belastung durch Etoricoxib jedoch verlangsamt zu werden. Somit bekräftigt diese Studie die Hypothese, dass die Symptome von DOMS durch die Einnahme von 
NSAR über einen längeren Zeitraum bestehen bleiben und der Regenerationsprozess verzögert wird. Eine mögliche Interpretation dieser Ergebnisse ist, dass die akute Entzündungsreaktion im Rahmen von DOMS nicht nur in der Entstehung des Muskelschmerzes eine wichtige Rolle spielt, sondern auch für den Regenerationsprozess der Muskulatur essentiell ist.

Auf Grundlage dieser Erkenntnisse sollte von einer unkritischen NSAR-Einnahme bei Muskelschmerzen nach starker sportlicher Belastung oder nach ungewohnten Alltagsaktivitäten wie z. B. Schneeschippen im Winter abgeraten werden. Eine durch die antientzündliche Wirksamkeit der NSAR bedingte mögliche Verzögerung der muskulären Regeneration könnte den scheinbar schmerzlindernden Effekt überwiegen. Diese Hypothese ist offensichtlich weiterhin spekulativ und muss durch weitere Studien mit hochpotenten NSAR bestätigt werden.

Zudem sollten in der Methodik nachfolgender Studien weitere Faktoren berücksichtigt werden, welche die Ergebnisse möglicherweise beeinflussen könnten. Es ergaben sich Hinweise darauf, dass bei Studien mit einer kürzeren Nachbeobachtungszeit, einer weniger häufigen Medikamentenapplikation und der Gabe eines schwächer antientzündlich wirksamen NSAR, ein möglicherweise falsch positiver Behandlungseffekt beobachtet werden könnte. Auch in folgenden Studien sollte die Nachbeobachtungsphase einen Zeitraum von einigen Tagen mit einschließen und die Effektbestimmung sowohl in der frühen Phase, als auch in einer späteren Phase nach exzentrischer Belastung erfolgen, um die scheinbar ambivalenten Behandlungseffekte von NSAR bei DOMS erfassen zu können.

In der Studie wurden physiologisch relevante, altersbezogene Unterschiede beobachtet. Ältere Erwachsene klagten nach der exzentrischen Belastung über signifikant weniger Muskelschmerz als jüngere Erwachsene. Zudem nahm der Regenerationsprozess der Muskulatur bei älteren Erwachsenen nach exzentrischer Belastung signifikant mehr Zeit in Anspruch als bei jüngeren Erwachsenen. Diese altersspezifischen Unterschiede sollten in nachfolgenden Studien, u. a. bei der Planung der Studienpopulation, beachtet werden. 


\section{LITERATURVERZEICHNIS}

Arendt-Nielsen, L., Weidner, M., Batholin, D., \& Rosetzsky, A. (2007). A Double-Blind Randomized Placebo Controlled Parallel Group Study Evaluating the Effects of Ibuprofen and Glucosamine Sulfate on Exercise Induced Muscle Soreness. Journal of Musculoskeletal Pain, 15(1):21-28.

Armstrong, R. (1990). Initial events in exercise-induced muscular injury. Medicine and Science in Sports and Exercise, 22(4):429-435.

Asmussen, E. (1956). Observations on experimental muscle soreness. Acta Rheumatologica Scandinavica, 2(2):109-116.

Baldwin, A., Stevenson, S., \& Dudley, G. (2001). Nonsteroidal Anti-Inflammatory Therapy After Eccentric Exercise in Healthy Older Individuals. Journal of Gerontology, 56(8):510513.

Barlas, P., Craig, J., Robinson, J., Walsh, D., Baxter, G., \& Allen, J. (2000). Managing Delayed-Onset Muscle Soreness: Lack of Effect of Selected Oral Systemic Analgesics. Archives of Physical Medicine and Rehabilitation, 81(7):966-972.

Basler, H., Grassl, C., Griessinger, N., Heinrich, R., Nehen, H., \& Siegel, N. (1999). Schmerz im Alter, Grundlagen der schmerztherapeutischen Versorgung älterer Menschen. Puchheim: Lukon-Verlag.

Bobbert, M., Hollander, A., \& Huijing, P. (1986). Factors in delayed onset muscle soreness of man. Medicine and Science in Sports and Exercise, 18(1):75-81.

Böning, D. (2002). Muskelkater. Deutsches Ärzteblatt, 99(6):372-375.

Böning, D. (2011). Wenn der Muskel exzentrisch wird - Energierückgewinnung des Muskels beim Bremsen. Deutsche Zeitschrift für Sportmedizin 62(3):55-56.

Bottas, R., Nicol, C., Komi, P., \& Linnamo, V. (2009). Adaptive Changes in motorcontrol of rhythmic movement after maximal eccentric actions. Journal of Electromyographic Kinesiology, 19(2):347-356.

Bourgeois, J., MacDougall, D., MacDonald, J., \& Tarnopolsky, M. (1999). Naproxen does not alter indices of muscle damage in resistance-exercise trained men. Medicine and Science in Sports and Exercise, 31(4):4-9.

Brunello, E., Reconditi, M., Elangovan, R., Linari, M., Sun, Y., Narayanan, T., Panine, P., Piazzesi, G., Irving, M., \& Lombardi, V. (2007). Skeletal muscle resists stretch by rapid binding of the second motor domain of myosin to actin. National Academy of Sciences, 104(50):20114-20119.

Cannavino, C., Abrams, J., Palinkas, L., Saglimbeni, A., \& Bracker, M. (2003). Efficacy of Transdermal Ketoprofen for Delayed Onset Muscle Soreness. Clinical Journal of Sports Medicine, 13(4):200-208. 
Cheung, K., Hume, P., \& Maxwell, L. (2003). Delayed onset muscle soreness - Treatment strategies and performance factors. Sports Medicine, 33(2):145-164.

Choi, S., Lim, J., Nibaldi, E., Phillips, E., Frontera, W., Fielding, R., \& Widrick, J. (2012). Eccentric contraction-induced injury to type I, Ila and Ila/llx muscle fibers of elderly adults. Age, 34(1):215-226.

Clancy, S., \& Clarkson, P. (1990). Immobilization during recovery from eccentric exercise. Medicine and Science of Sports and Exercise, 22(2):S37.

Clarkson, P., \& Hubal, M. (2002). Exercise-induced muscle damage in humans. Journal of Physical Medicine and Rehabilitation, 81(11):S52-S69.

Cleak, M., \& Eston, R. (1992). Muscle Soreness, swelling, stiffness and strength loss after intense eccentric exercise. British Journal of Sports Medicine, 26(4):267-272.

Cohen, J. (1988). Statistical Power Analysis for the Behavioral Sciences. Hillsdale: Routledge-Verlag.

Connolly, D., Sayers, S., \& McHugh, M. (2003). Treatment and prevention of delayed onset muscle soreness. Journal Of Strength And Conditional Research, 17(1):197-208.

Coudreuse, J., Dupont, P., \& Nicol, C. (2004). Delayed post effort muscle soreness. Annales de réadaptation et de médecine physique, 47(6):290-298.

Crameri, R., Aagaard, P., Qvortrup, K., Langberg, H., Olesen, J., \& Kjaer, M. (2001). Myofibre damage in human skeletal muscle: effects of electrical stimulation versus voluntary contraction. Journal of Physiology, 583(1):265-280.

Dallob, A., Hawkey, C., Greenberg, H., Wight, N., de Schepper, P., Waldman, S., Wong, P., DeTora, L., Gertz, B., Agrawal, N., Wagner, J., \& Gottesdiener, K. (2003). Characterization of etoricoxib, a novel, selective COX-2 inhibitor. Journal of Clinical Pharmacology, 43(6):573-85.

Dartnall, T., Nordstrom, M., \& Semmler, J. (2008). Motor unit synchronization is increased in biceps brachii after exercise-induced damage to elbow flexor muscles. Journal of Neurophysiology, 99(2):1008-1019.

de Vries, H. (1966). Quantitative EMG investigation of the spasm theory of muscle pain. American Journal of Physiological Medicine, 45:119-134.

Dedrick, M., \& Clarkson, P. (1990). The effects of eccentric exercise on motor performance in young and older women. European Journal of Applied Physiology, 60(3):183-186.

Donnelly, A., Maughan, R., \& Whiting, P. (1990). Effects of ibuprofen on exercise-induced muscle soreness and indices of muscle damage. British Journal of Sports Medicine, 24(3):191-195. 
Donnelly, A., McCormick, K., Maughan, R., Whiting, P., \& Clarkson, P. (1988). Effects of a non-steroidal anti-inflammatory drug on delayed onset muscle soreness and indices of damage. British Journal of Sports Medicine 22(1):35-38.

Dudley, G., Czerkawski, J., Meinrod, A., Gillis, G., Baldwin, A., \& Scarpone, M. (1997). Efficacy of Naproxen Sodium for Exercise-Induced Dysfunction Muscle Injury and Soreness. Clinical Journal of Sports Medicine, 7(1):3-10.

Dundon, J., Cirillo, J., \& Semmler, J. (2008). Low-frequency fatigue and neuromuscular performance after exercise-induced damage to elbow flexor muscles. Journal of Applied Physiology, 105(4):1146-1155.

Faulkner, J., Brooks, S., \& Opiteck, J. (1993). Injury to skeletal muscle fibres during contractions: Conditions of occurence and prevention. Physical Therapy, 73(12):911-921.

Fielding, R., Manfredi, T., Ding, W., Fiatarone, M., Evans, W., \& Cannon, J. (1993). Acute phase response in exercise. III. Neutrophil and IL-1ß accumulation in skeletal muscle. American Journal of Physiology, 256(1):R166-R172.

Fridén, J., \& Lieber, R. (2001). Eccentric exercise-induced injuries to contractile and cytoskeletal muscle fibre components. Acta Physiologica Scandinavia, 171(3):321-326.

Fridén, J., \& Lieber, R. (1992). Structural and mechanical basis of exercise induced muscle injury. Medicine and Science in Sports and Exercise, 24(5):521-530.

Fridén, J., Sfakianos, P., \& Hargens, A. (1986). Delayed muscle soreness and intramuscular fluid pressure: comparison between eccentric and concentric load. Journal of Applied Physiology, 61(6):2175-2179.

Fridén, J., Sjoström, M., \& Ekblom, B. (1983). Myofibrillar damage following intense eccentric exercise in man. International Journal of Sports Medicine, 4(3):170-176.

Furst, D. (1999). Pharmacology and efficacy of cyclooxygenase (COX) inhibitors. American Journal of Medicine, 107(6A):18S-26S.

Gibson, S., \& Helme, R. (2001). Age-related differences in pain perception and report. Clinics in Geriatric Medicine, 17(3):433-456.

Gissel, H. (2000). $\mathrm{Ca}^{2+}$ accumulation and cell damage in skeletal muscle during low frequency stimulation. European Journal of Applied Physiology, 83(2):175-180.

Grossmann, J., Arnold, B., Perrin, D., \& Kahler, D. (1995). Effect of Ibuprofen on pain, decreased range of motion and decreased strength associated with delayed onset muscle soreness of the ellbow flexors. Journal of Sport Rehabilitation, 4:253-263.

Harkins, S., \& Price, D. (1992). Assessment of pain in the elderly.

Hasson, S., Daniels, J., Divine, J., Niebuhr, B., Richmond, S., Stein, P., \& Williams, J. (1993). Effect of ibuprofen on muscle soreness, damage and performance: a preliminary investigation. Medical Science Sports and Exercise, 25(9):9-17. 
Hedayatpour, N., Hassanlouei, H., Arendt-Nielsen, L., Kersting, U., \& Falla, D. (2011). Delayed onset muscle soreness alters the response to postural perturbations. Medicine and Science in Sports and Exercise, 43(6):1010-1016.

Hertel, J. (1997). The Role of Nonsteroidal Anti-Inflammatory Drugs in the Treatment of Acute Soft Tissue Injuries. Journal of Athletic Training, 32(4):350-358.

Hough, T. (1902). Ergographic studies in muscular soreness. American Journal of Physiology, 7(1):76-92.

Howatson, G., \& van Someren, K. (2008). The Prevention and Treatment of ExerciseInduced Muscle Damage. Sports Medicine, 38(6):483-503.

Howell, J., Conatser, R., Chleboun, G., Karapondo, D., \& Chila, A. (1998). The Effect of Nonsteroidal Anti-Inflammatory Drugs on Recovery from Exercise-Induced Muscle Injury: 2. Ibuprofen. Journal of Musculoskeletal Pain, 6(4):69-83.

Howell, J., Conatser, R., Chleboun, G., Karapondo, D., \& Chila, A. (1998). The Effect of Nonsteroidal Anti-Inlfammatory Drugs on Recovery from Exercise-Induced Muscle Injury: 1. Flurbiprofen. Journal of Musculoskeletal Pain, 6(4):59-68.

Hyldahl, R., Keadle, J., Rouzier, P., Pearl, D., \& Clarkson, P. (2010). Effects of Ibuprofen Topical Gel on Muscle Soreness. Medicine and Science in Sports and Exercise, 42(3):614-621.

Jones, D., Newham, D., Round, J., \& Tolfree, S. (1986). Experimental human muscle damage: morphological changes in relation to other indices of damage. Journal of Physiology, 375(1):435-448.

Kaariainen, M., Jarvinen, T., Jarvinen, M., Rantanen, J., \& Kalimo, H. (2000). Relation betwenn myofibers and connective tissue during muscle injury repair. Scandinavian Journal of Medicine in Science and Sports, 10(6):332-337.

Koch, G. (1972). The use of non-parametric methods in the statistical analysis of the twoperiod changeover design. Biometrics, 28:577-84.

Kuipers, H., Keizer, H., Verstappen, F., \& Costill, D. (1985). Influence of a ProstaglandinInhibiting Drug on Muscle Soreness After Eccentric Work. International Journal of Sports Medicine, 6(6):336-339.

Lachin, J. (1992). Some large-sample distribution-free estimators and tests for multivariate partially incomplete data from two populations. Statistics in Medicine, 11(9):1151-1170.

Lanier, A. (2003). Use of Nonsteroidal Anti-Inflammatory Drugs Following ExerciseInduced Muscle Injury. Sports Medicine, 33(3):177-186.

Lapointe, B., Frémont, P., \& Côté, C. (2002). Adaption to lengthening contractions is independent of voluntary muscle recruitement but relies on inflammation. American 
Journal of Physiology - Regulatory, Integrative and Comparative Physiology, 282(1):323329.

Lavender, A., \& Nosaka, K. (2008). A light load eccentric exercise confers protection against a subsequent bout of more demanding eccentric exercise. Journal of Science and Medicine in Sport, 11(3):291-298.

Lecomte, J., Lacroix, V., \& Montgomery, D. (1998). A Randomized Controlled Trial of the Effect of Naproxen on Delayed Onset Muscle Soreness and Muscle Strength. Clinical Journal of Sports Medicine, 8(2):82-87.

Lexell, J., Taylor, C., \& Sjostrom, M. (1988). What is the cause of the ageing atrophy? Total number, size and proportion of different fibre types studied in whole vastus lateralis muscle from 15- to 83-year-old men. Journal of Neurological Sciences, 84(2-3):275-294.

Lieber, R., Woodburn, T., \& Fridén, J. (1991). Muscle damage induced by eccentric contractions of 25\% strain. Journal of Applied Physiology, 70(6):2498-2507.

Maclntyre, D., Reid, W., \& McKenzie, D. (1995). Delayed muscle soreness. The inflammatory response to muscle injury and its clinical implications. Sports Medicine, 20(1):24-40.

Maclntyre, D., Reid, W., Lyster, D., \& McKenzie, D. (2000). Different effects of strenous eccentric exercise on the accumulation of neutrophils in muscle in women and men. European Journal of Applied Physiology, 81(1):47-53.

Mackey, A., Mikkelsen, U., Magnusson, S., \& Kjaer, M. (2012). Rehabilitation of muscle after injury - the role of anti-inflammatory drugs. Scandinavian Journal of Medicine and Science in Sports, 8-14.

Malm, C., Nyberg, P., Engström, M., Sjödin, B., Lenkei, R., Ekblom, B., et al. (2000). Immunological changes in human sceletal muscle and blood after eccentric exercise and multiple biopsies. Journal of Physiology, 529(1):243-262.

Manfredi, T., Fielding, R., O'Reilly, K., Meredith, C., Lee, H., \& Evans, W. (1991). Plasma creatine kinase activity and exercise-induced muscle damage in older men. Medicine and Science in Sports and Exercise, 23(9):1028-1034.

McHugh, M., Connolly, D., Eston, R., \& Gleim, G. (1999). Exercise-induced muscle damage and potential mechanisms for the repeated bout effect. Sports Medicine, 27(3):157-170.

Miles, M., \& Clarkson, P. (1994). Exercise-induced muscle pain, soreness, and cramps. Journal of Sports Medicine and Physical Fitness, 34(3):203-216.

Mishra, D., Friden, J., Schmitz, M., \& Lieber, R. (1995). Anti-inflammatory medication after muscle injury. A treatment resulting in short-term improvement but subsequent loss of muscle function. The Journal of Bone \& Joint Surgery, 7:1510-1519. 
Morgan, D., \& Allen, D. (1999). Early events in stretch-induced muscle damage. Journal of Applied Physiology, 87(6):2007-2015.

MSD. (2011). Fachinformation Arcoxia. MSD.

Newham, D., Jones, D., \& Clarkson, P. (1987). Repeated high force eccentric exercise: effects on muscle pain and damage. Journal of Applied Physiology, 63(4):1381-1386.

Newham, D., McPhail, I., Mills, K., \& Edwards, R. (1983). Ultrastructural changes after concentric and eccentric contractions of human muscle. Journal of Neurological Sciences, 61(1):109-122.

Newham, D., Mills, K., \& Quigley, R. (1982). Muscle pain and tenderness after exercise. Australien Journal Of Sport Medicine and Exercise Science, 14:129-131.

Nieman, D., Henson, D., Dumke, C., Oley, K., McAnulty, S., Davis, J., et al. (2006). Ibuprofen use, endotoxemia, inflammation and plasma cytokines during ultramarathon competition. Brain, Behaviour and Immunity, 20(6):578-584.

Noonan, T., \& Garrett, W. (1992). Injuries at the myotendinous junction. Clinical Sports Medicine, 11(4):783-806.

Nosaka, K., Sakamoto, K., Newton, M., \& Sacco, P. (2001). How long does the protective effect on eccentric-induced muscle damage last? Medicine and Science in Sports and Exercise, 33(9):1490-1495.

O'Grady, M., Hackney, A., Schneider, K., Bossen, E., Steinberg, K., Douglas, J., Murray, W., \& Watkins, W. (2000). Diclofenac sodium (Voltaren) reduced exercise-induced injury in human skeletal muscle. Medicine and Science in Sports and Exercise, 32(7):11911196.

Paulsen, G., Egner, I., Drange, M., Langberg, H., Benestad, H., Fjeld, J., Hallen, J., \& Raastad, T. (2010). A COX-2 inhibitor reduces muscle soreness, but does not influence recovery and adaption after eccentric exercise. Scandinavian Journal of Medicine and Science in Sports, 20(1):195-207.

Philippou, A., Bogdanis, G., Maridaki, M., Halapas, A., Sourla, A., \& Koutsilieris, M. (2009). Systematic cytokine following exercise-induced muscle damage in humans. Clinical Chemical Labaratory Medicine, 47(6):777-782.

Pizza, F., Cavender, D., Stockard, A., Baylies, H., \& Beighle, A. (1999). Anti-Inflammatory Doses of Ibuprofen: Effect on Neutrophils and Exercise-Induced Muscle Injury. International Journal of Sports Medicine, 20:98-102.

Ploutz-Snyder, L., Giamis, E., Formikell, M., \& Rosenbaum, A. (2001). Resistance Training Reduces Susceptibility to Eccentric Exercise-Induced Muscle Dysfunction in Older Women. Journal of Gerontology, 56(9):384-390. 
Prisk, V., \& Huard, J. (2003). Muscle injuries and repair: The role of prostaglandins and inflammation. Histology and Histopathology, 56(9):1243-1256.

Rahnama, N., Rahmani-Nia, F., \& Ebrahim, K. (2005). The isolated and combined effects of selected physical activity and ibuprofen on delayed-onset muscle soreness. Journal of Sports Sciences, 23(8):843-850.

Rantanen, J., Ranne, J., Hurme, T., \& Kalimo, H. (1995). Satellite cell proliferation and expression of myogenin and desmin in regenerating skeletal muscle. Laboratory Investigation, 72(3):341-347.

Riasati, S., Moghadasi, M., Torkfar, A., Shirazinejad, R., \& Arvin, H. (2010). Aspirin may be an effective treatment for exercise-induced muscle soreness. Brazilian Journal of Biomotricity, 4(3):206-213.

Röhrig, B., du Prel, J., Wachtlin, D., Kwiecien, R., \& Blettner, M. (2010). Sample size calculation in clinical trials - part 13 of a series on evaluation of scientific publications. Deutsches Ärzteblatt International, 107(31-32):552-556.

Rother, M., \& Rother, I. (2011). Placebo controlled, crossover validation study of oral ibuprofen and topical hydrocortisone-21-acetate for a model of ultraviolet $B$ radiation (UVR)-induced pain and inflammation. Journal of Pain Research, 4:357-363.

Rother, M., Seidel, E., Clarkson, P., Mazgareanu, S., Vieri, U., \& Rother, I. (2009). Efficacy of epicutaneous Diractin (ketoprofen in Transfersome) for the treatment of pain related to eccentric muscle contractions. Drug Design, Development and Therapy, 3:1-7.

Rother, M., Seidel, E., Fischer, A., \& Rother, I (2011). Validation Study for a Model of Acute Muscle Pain. Physikalische Medizin, Rehabilitationsmedizin, Kurortmedizin, 22(4):199-202.

Ryan, G., \& Majno, G. (1977). Acute inflammation. American Journal Of Pathology, 86(1):185-264.

Sayers, P., Knight, C., Clarkson, P., van Wegen, E., \& Kamen, G. (2001). Effect of ketoprofen on muscle function and SEMG activity after eccentric exercise. Medicine and Science in Sports and Exercise, 33(5):702-710.

Schwane, J., Hatrous, B., \& Johnson, S. (1983). Is lactic acid related to delayed onset muscle soreness? Physical Sports Medicine, 11(3):124-127.

Schwartz, J., Dallob, A., Larson, P., Laterza, O., Miller, J., Royalty, J., Snyder, K., Chappell, D., Hilliard, D., Flynn, M., Cavanaugh, P., \& Wagner, J. (2008). Comparative Inhibitory Activity of Etoricoxib, Celecoxib and Diclofenac on COX-2 Versus COX-1 in Healthy Subjects. The Journal of Clinical Pharmacology, 48(6):745-754.

Seichert, N. (2003). Besonderheiten der exzentrischen Muskelaktivität - Konsequenzen für die Rehabilitation. Österreichische Zeitschrift für Physikalische Medizin und Rehabilitation, 13(1):10. 
Seidel, E., Rother, M., Hartmann, J., Rother, I., Schaaf, T., Winzer, M., Fischer, A., Regenspurger, K. (2012). Eccentric Exercise and Delayed Onset Muscle Soreness (DOMS) - an Overview. Physikalische Medizin, Rehabilitationsmedizin, Kurortmedizin, 22(2):57-63.

Semark, A., Noakes, T., Gibson, A., \& Lambert, M. (1999). The effect of a prophylactic dose of flurbiprofen on muscle soreness and sprinting performance in trained subjects. Journal of Sports Science, 17(3):197-203.

Semmler, J., Tucker, K., Allen, T., \& Proske, U. (2007). Eccentric exercise increases EMG amplitude and force fluctuations during submaximal contractions of elbow flexor muscles. Journal of Applied Physiology, 103(3):979-989.

Shellock, F., Fukunaga, T., \& Mink, J. (1991). Exertional muscle injury: Evaluation of concentric versus eccentric actions with serial MR imaging. Radiology, 179(3):659-664.

Sjödin, B., Hellsten Westing, Y., \& Apple, F. (1990). Biochemical mechanisms for oxigen free radical formation during exercise. Sports Medicine, 10(4):236-254.

Smith, L. (1991). Acute inflammation: the underlying mechanism in delayed onset muscle soreness? Medicine and Science in Sports and Exercise, 23(5):542-551.

Soltow, Q., Betters, J., Sellman, J., Lira, V., Long, J., \& Criswell, D. (2006). Ibuprofen Inhibits Skeletal Muscle Hypertrophy in Rats. Medicine and Science in Sports and Exercise, 38(5):840-846.

Stauber, W., Clarkson, P., \& Fritz, V. (1990). Extracellular matrix disruption and pain after eccentric muscle action. Journal of Applied Physiology, 69(3):868-874.

Steinmann, H., \& Allwang, D. (2008). Verletzungen im Sport: vermeiden - behandeln therapieren. München: Urban \& Fischer-Verlag.

Stone, M., Merrick, M., Ingersoll, C., \& Edwards, J. (2002). Preliminary Comparison of Bromelain and Ibuprofen for Delayed Onset Muscle Soreness Management. Clinical Journal of Sport Medicine, 12(6):373-378.

Tofas, T., Jamurtas, A., Fatouros, I., Nikolaidis, M., Koutedakis, Y., Sinouris, E., Papageorgakopoulou, N., \& Theocharis, D. (2008). Plyometric exercise increases serum indices of muscle damage and collagen breakdown. Journal of Strength and Conditioning Research, 22(2):490-496.

Tokmakidis, S., Kokkinidis, E., Smilios, I., \& Doudas, H. (2003). The Effects of Ibuprofen on Delayed Muscle Soreness and Muscular Performance After Eccentric Exercise. Journal of Strength and Conditioning Research, 17(1):53-59.

Trappe, T., Fluckey, J., White, F., Lambert, C., \& Evans, W. (2001). Skeletal Muscle $\mathrm{PGF}_{2 \mathrm{a}}$ and $\mathrm{PGE}_{2}$ in Response to Eccentric Resistance Exercise: Influence of Ibuprofen and Acetaminphen. The Journal of Clinical Endocrinology \& Metabolism, 86(10):50675070 . 
Trappe, T., White, F., Lambert, C., Cesar, D., Hellerstein, M., \& Evans, W. (2001). Effect of ibuprofen and acetaminophen on postexercise muscle protein synthesis. American Journal of Physiology - Endocrinology and Metabolism, 282(3):551-556.

Twist, C., \& Eston, R. (2005). The effects of exercise-induced damage on maximal intensity intermittent exercise performance. European Journal of Applied Physiology, 94(5):652-658.

van Hecken, A., Schwartz, J., Depre, M., de Lepeleire, I., Dallob, A., Tanaka, W., Wynants, K., Buntinx, A., Arnout, J., Wong, P., Ebel, D., Gertz, B., \& de Schepper, D. (2000). Comparative inhibitory activity of rofecoxib, meloxicam, diclofenac, ibuprofen and naproxen on COX-2 versus COX-1 in healthy subjects. The Journal of Clinical Pharmacology, 40(10):1109-1120.

Wallace, J. (1999). Distribution and expression of cyclooxygenase (COX) isoenzymes, their physiological roles, and the categorization of nonsteroidal anti-inflammatory drugs (NSAIDs). American Journal Of Medicine, 107(6A):11S-17S.

Weineck, J. (2007). Optimales Training: Leistungsphysiologische Trainingslehre unter besonderer Berücksichtigung des Kinder- und Jugendtrainings. Balingen: Spitta-Verlag.

Wellek, S., \& Blettner, M. (2012). On the proper use of the crossover design in clinical trials: part 18 of a series on evaluation of scientific publications. Deutsches Ärzteblatt, 109(15):276-81.

Wietoska, B., \& Böning, D. (1979). Was ist eigentlich Muskelkater? Deutsche Zeitschrift für Sportmedizin, 30:395-401. 


\section{ANHANG}

\section{Anh. 1: Zustimmung der LÄK Thüringen zur Durchführung der Studie}

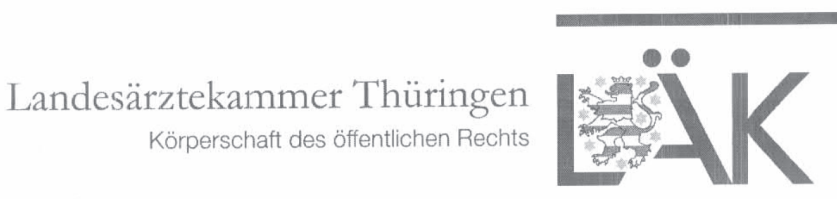

Ethikkommission

\begin{tabular}{|c|c|c|}
\hline \multirow[b]{2}{*}{ Landesărztekammer Thüringen · Postfach $100740 \cdot 07707$ Jena } & \multirow{2}{*}{\multicolumn{2}{|c|}{ Im Semmicht $33 \cdot 07751$ Jena-Maua }} \\
\hline & & \\
\hline \multirow{6}{*}{$\begin{array}{l}\text { Herrn } \\
\text { Dr. med. Matthias Rother } \\
\text { X-pert Med GmbH } \\
\text { Lärchenstraße 3-3a } \\
82166 \text { Gräfelfing }\end{array}$} & Telefon: & $03641614-0$ \\
\hline & Internet: & www. laek-thueringen.de \\
\hline & & \\
\hline & Ihr Ansprechpartner: & Frau Köhler \\
\hline & Durchwahl: & 03641 614-201 \\
\hline & Bereichsfax: & 03641 614-209 \\
\hline Thr Zeichen: & Bereichs-Email: & ethikkommission $@$ a laek-thueringen.de \\
\hline & $\begin{array}{l}38810 / 2010 / 126 \\
\text { (Bitte immer angeben) }\end{array}$ & 16. Dezember 2010 \\
\hline
\end{tabular}

vorab per Fax: 089858360926

Randomisierte, doppelblinde, plazebo-kontrollierte, cross-over Studie zur Beurteilung von Etoricoxib für die Behandlung von Muskelschmerz und Entzündung nach exzentrischer Belastung

Studien-Nr.: XPM-032

EudraCT-Nr.: 2010-023493-40

Sehr geehrter Herr Dr. Rother,

die Ethik-Kommission der Landesärztekammer Thüringen ist die zuständige Ethik-Kommission für die o. g. Studie. Die Ethik-Kommission hat am 14. Dezember 2010 die Studie umfassend - teilweise zusammen mit Ihnen und Frau Dipl.-Med. Ilka Rother - beraten. Dabei wurden die in der Anlage 1 erwähnten Unterlagen berücksichtigt.

Die Ethik-Kommission erteilt eine

zustimmende Bewertung.

Begründung:

Ablehnungsgründe gemäß $§ 42$ Absatz 1 Satz 7 Arzneimittelgesetz lagen nicht vor.

Die Ethik-Kommission weist darauf hin, dass die ärztliche und juristische Verantwortung bei den jeweiligen Prüfärzten verbleibt.

An der Beratung und Beschlußfassung haben die in der Anlage 2 aufgeführten Mitglieder der hiesigen Ethik-Kommission teilgenommen. Es wird bestätigt, dass Prüfärzte, die an der o. g. Studie beteiligt sind, nicht an der Abstimmung teilgenommen haben.

\begin{tabular}{llll}
\hline Deutsche Apotheker- und Ärztebank Erfurt & & \\
Konto-Nr.: & 0003101401 & BLZ: & 30060601 \\
IBAN: & DE 40300606010003101401 & BIC: & DAAEDEDD
\end{tabular}




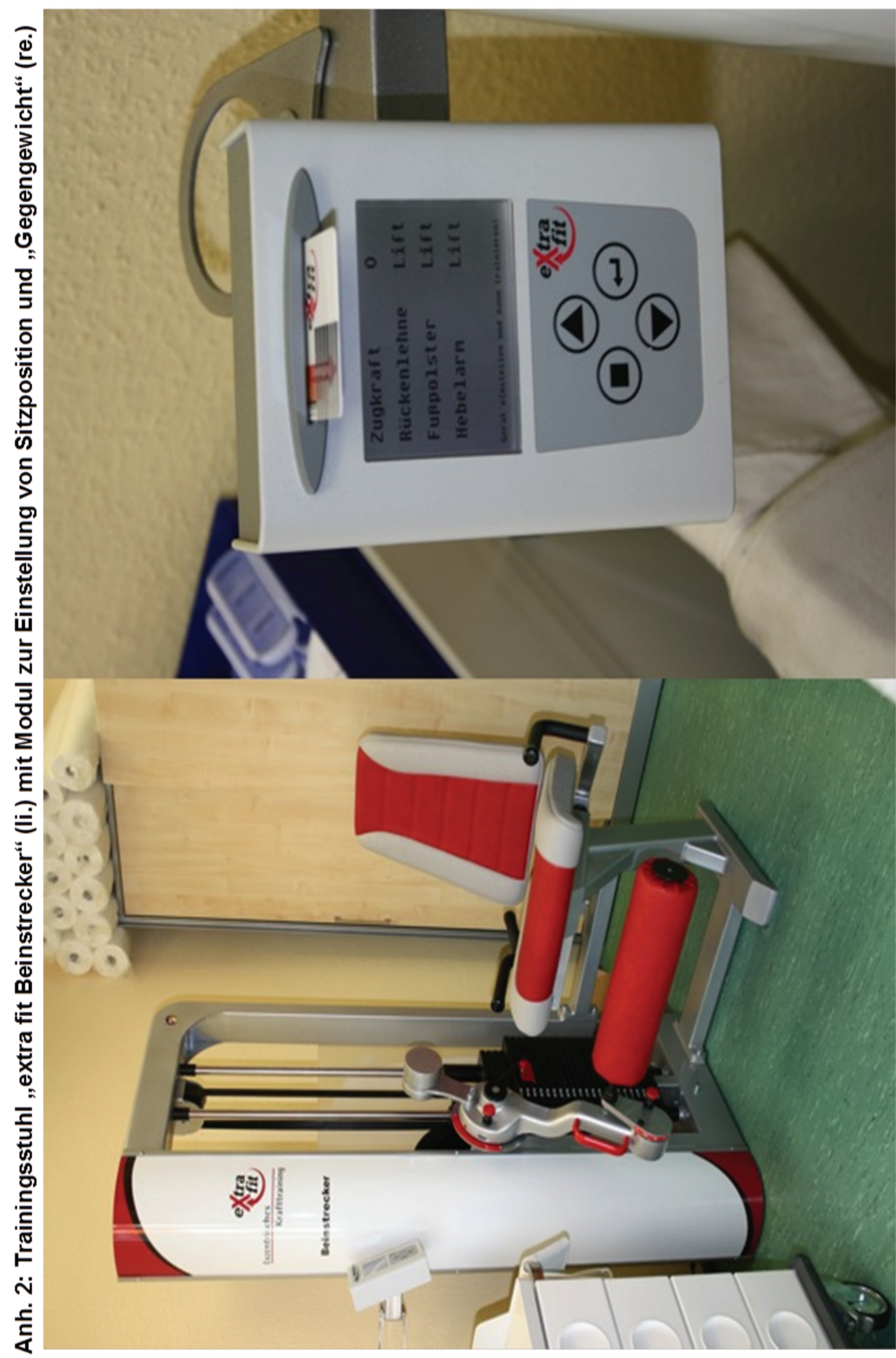




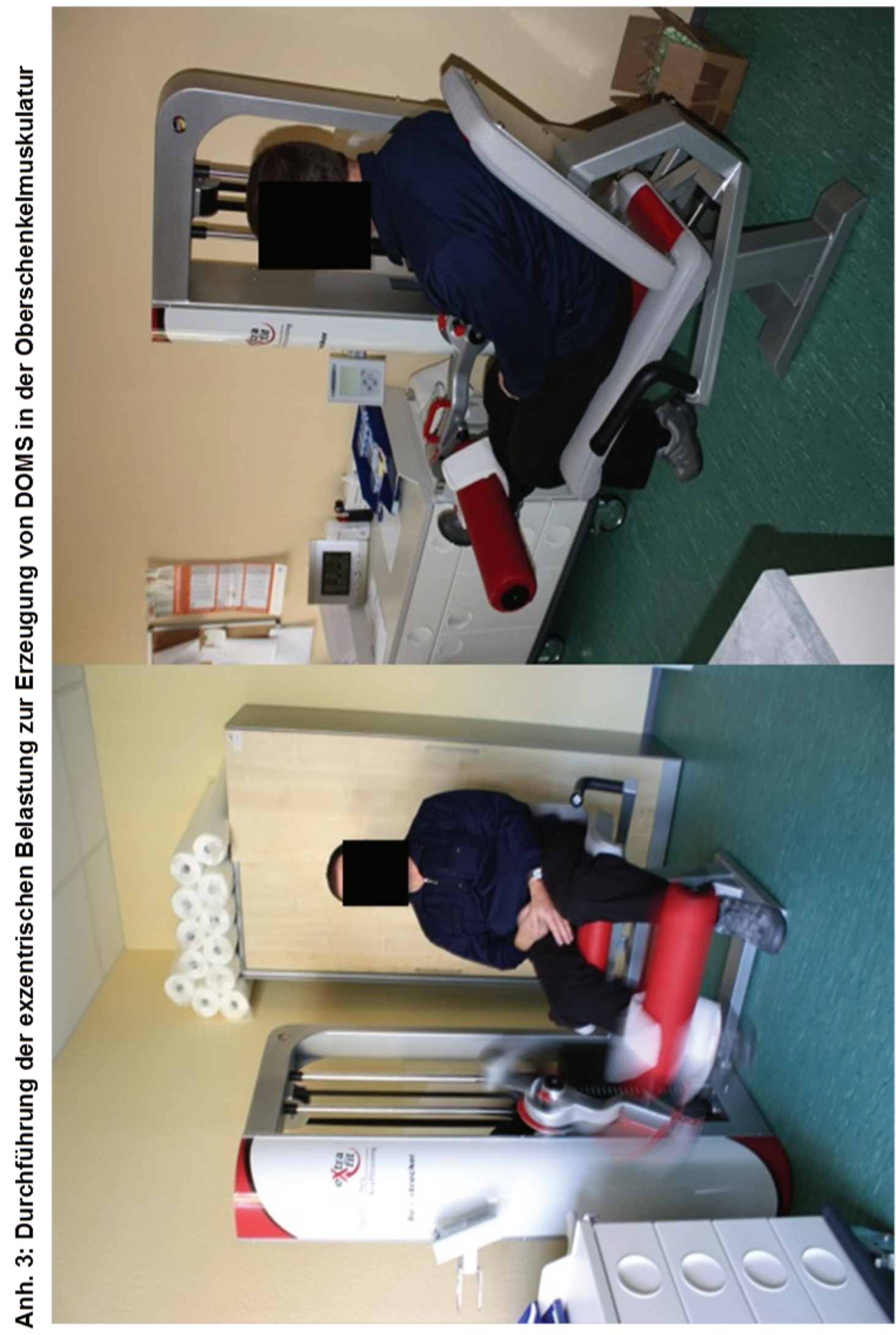




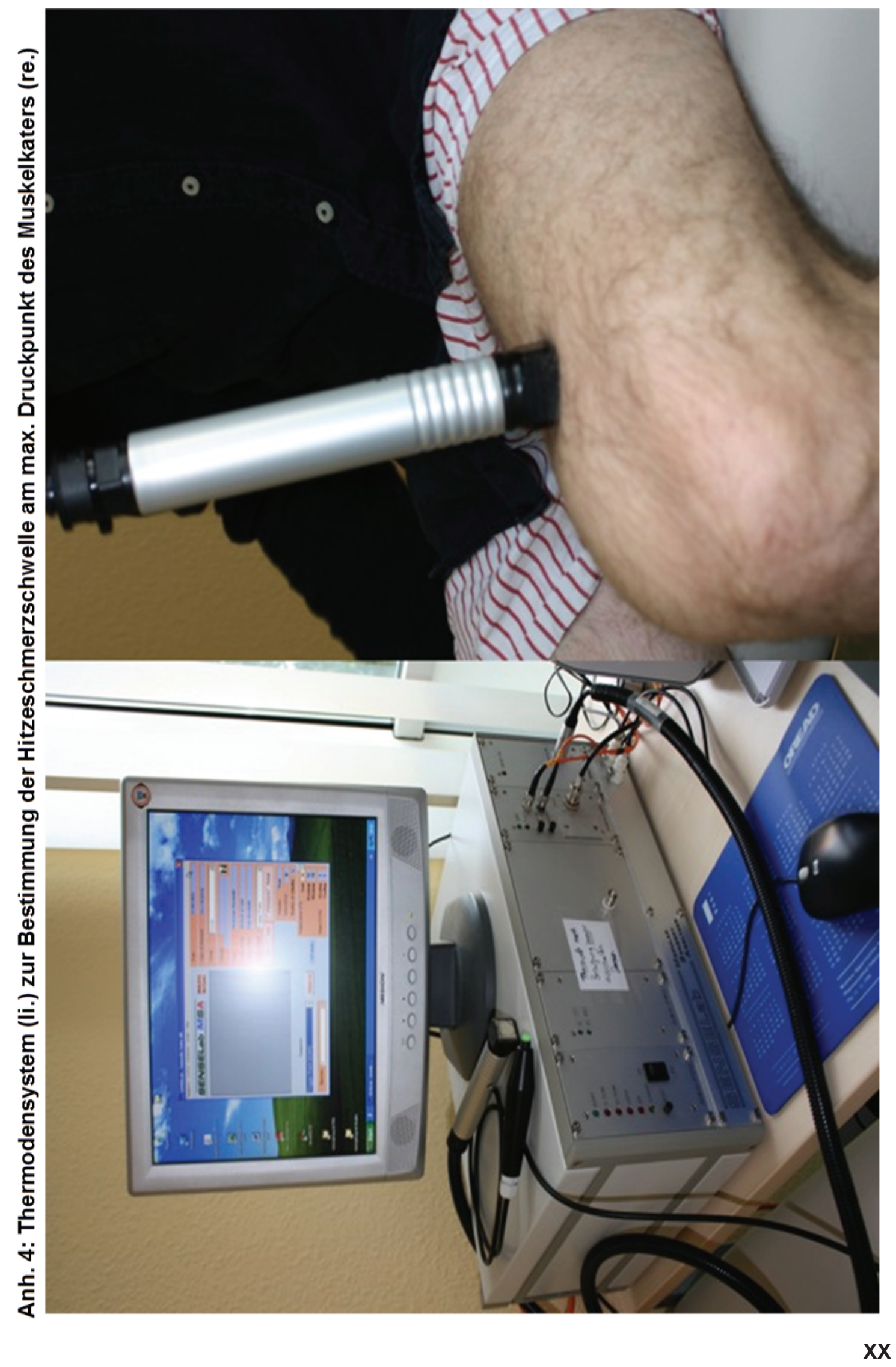




\section{LEBENSLAUF}

PERSONALIEN

Name: $\quad$ Steffen Koll

Geburtsdatum: $\quad 04.12 .1983$

Geburtsort: Memmingen

Familienstand: $\quad$ ledig

SCHULISCHER WERDEGANG

$1996-2003 \quad$ Gymnasium

29.07.2003 Abitur

UNIVERSITÄRER WERDEGANG

WS $2004 \quad$ Beginn des Studiums der Medizin

an der Friedrich-Schiller-Universität Jena

25.09.2006 1. Abschnitt der Ärztlichen Prüfung

13.12.2011 2. Abschnitt der Ärztlichen Prüfung

22.12.2011 Approbation als Arzt

BERUFLICHER WERDEGANG

$01.2012-08.2012 \quad$ Assistenzarzt am Zentrum für Physikalische und Rehabilitative Medizin des Sophien- und Hufeland-Klinikums Weimar

seit 11.2012

Assistenzarzt in der Klinik für Manuelle Medizin und

Konservative Orthopädie der Sana Kliniken Sommerfeld 


\section{DANKSAGUNG}

Diese Arbeit ist während meiner Tätigkeit am Zentrum für Physikalische und Rehabilitative Medizin des Sophien- und Hufeland-Klinikums in Weimar entstanden.

Für die großzügige Überlassung des Themas und die ausgezeichneten Möglichkeiten es zu bearbeiten möchte ich in erster Linie meinem damaligen Chefarzt Herrn Prof. Dr. med. E. J. Seidel herzlich danken. Für die zahlreichen Hinweise und Ratschläge in wissenschaftlicher als auch in beruflicher und privater Hinsicht bin ich sehr dankbar.

Einen ganz besonderen Dank möchte ich Herrn Dr. med. habil. M. Rother aussprechen, der es mir ermöglichte an der Studie mitzuwirken und mich stets mit Großzügigkeit und Geduld unterstützte.

Ebenso danke ich Herrn Prof. Dr. med. U. Smolenski für die Annahme des Themas, die vielen sachlichen Hinweise und die ausgezeichnete Betreuung der Arbeit. Für die Durchsicht der Arbeit und die vielen Hinweise, die zur Vollendung derselben notwendig waren, danke ich meinen Freunden Sören Nagel, Lydia Ranke, Sebastian Schütte, Alexander Fischer und meinem Bruder Friedemann Koll.

Ein außergewöhnlicher Dank gilt meinen Eltern und meiner Familie für die immerwährende Unterstützung. 


\section{EHRENWÖRTLICHE ERKLÄRUNG}

Hiermit erkläre ich, dass mir die Promotionsordnung der Medizinischen Fakultät der Friedrich-Schiller-Universität bekannt ist,

ich die Dissertation selbst angefertigt habe und alle von mir benutzten Hilfsmittel, persönlichen Mitteilungen und Quellen in meiner Arbeit angegeben sind,

mich folgende Personen bei der Auswahl und Auswertung des Materials sowie bei der Herstellung des Manuskripts unterstützt haben: Prof. Dr. med. E. J. Seidel und Dr. med. habil. M. Rother,

die Hilfe eines Promotionsberaters nicht in Anspruch genommen wurde und dass Dritte weder unmittelbar noch mittelbar geldwerte Leistungen von mir für Arbeiten erhalten haben, die im Zusammenhang mit dem Inhalt der vorgelegten Dissertation stehen,

dass ich die Dissertation noch nicht als Prüfungsarbeit für eine staatliche oder andere wissenschaftliche Prüfung eingereicht habe und

dass ich die gleiche, eine in wesentlichen Teilen ähnliche oder eine andere Abhandlung nicht bei einer anderen Hochschule als Dissertation eingereicht habe.

Berlin, den 26.03.2013 